\title{
Internal kinematics and dynamical models of dwarf spheroidal galaxies around the Milky Way
}

\author{
Giuseppina Battaglia, ${ }^{\mathrm{a}, *}$, Amina Helmi ${ }^{\mathrm{b}}$, Maarten Breddels ${ }^{\mathrm{b}}$ \\ ${ }^{a}$ INAF - Astronomical Observatory of Bologna, via Ranzani 1, 40127 Bologna, I \\ ${ }^{b}$ Kapteyn Astronomical Institute, P.O. Box 800, 9700 AV Groningen, NL
}

\begin{abstract}
We review our current understanding of the internal dynamical properties of the dwarf spheroidal galaxies surrounding the Milky Way. These are the most dark matter dominated galaxies, and as such may be considered ideal laboratories to test the current concordance cosmological model, and in particular provide constraints on the nature of the dominant form of dark matter. We discuss the latest observations of the kinematics of stars in these systems, and how these may be used to derive their mass distribution. We tour through the various dynamical techniques used, with emphasis on the complementarity and limitations, and discuss what the results imply also in the context of cosmological models. Finally we provide an outlook on exciting developments in this field.
\end{abstract}

Keywords:

Galaxies: kinematics and dynamics, Galaxies: dwarf, dark matter, Local Group, Galaxies: stellar content.

\section{Introduction}

With absolute magnitudes ranging from $M_{V} \sim-9$ to $\sim-13.5$ and central surface brightness between $\mu_{0, V} \sim 22.5-27 \mathrm{mag} \operatorname{arcsec}^{-2}$, the "dwarf spheroidals" (dSphs) are the faintest and lowest surface brightness galaxies known to date, beaten only by the relatively recently discovered ultra-faint dwarf galaxies (UFDs).

\footnotetext{
${ }^{*}$ Corresponding author

Email address: gbattaglia@oabo.inaf.it (Giuseppina Battaglia)
} 
Although of dull appearance, dSphs reveal an unexpectedly complex stellar populations mix (for a recent review see Tolstoy et al., 2009) that makes them very useful laboratories for understanding star formation and chemical enrichment processes at the faint end of the galaxy luminosity function. In terms of their internal dynamics, they might well be key in constraining the nature of dark matter. Even though the very first measurement of the line-of-sight velocity dispersion of a dSph was based on just 3 carbon stars in Draco (Aaronson, 1983), it already hinted at a dynamical mass-to-light ratio about one order of magnitude larger than for globular clusters. Subsequent works have confirmed this result using larger samples that included red giant stars (e.g. Armandroff and Da Costa, 1986; Aaronson and Olszewski, 1987; Hargreaves et al., 1994a b, see also Sec. 2 and 3). If in dynamical equilibrium, dSphs have the highest mass-to-light ratios known to dat@? with $M / L \sim 100 \mathrm{~s}_{\odot} / \mathrm{L}_{\odot}$.

In the remainder of the Introduction we describe the latest observational surveys of the kinematics of dSphs, place these systems in a cosmological context and briefly discuss why most of these systems may be considered to be in dynamical equilibrium. In Sec. 2 we describe the determination of the kinematic properties of dSphs from spectroscopic samples of individual stars. In Sec. 3 we review the methods used to model the internal dynamics of spheroidal systems and discuss their application to dSphs around the Milky Way. We discuss possible future developments in Sec. 4, and in Sec. 5 we briefly summarize the current status of the field.

\subsection{Surveys of dwarf spheroidals around the Milky Way}

Determining the mass content of a system requires observations of the kinematics of suitable tracers. Since dSphs are devoid of a neutral interstellar medium, the only tracers available are stars. Because of their distance, todate all measurements of their internal kinematics are based on line-of-sight velocities. The stars accessible for spectroscopic observations with current facilities are resolved for systems within the Local Group, since there is no crowding because of the low surface brightness of these galaxies. In this review, we concentrate on the dwarf galaxies that are satellites of the Milky Way (MW, hereafter. We refer the reader to Walker, 2012, for a nice and

\footnotetext{
${ }^{1}$ While at face value UFDs exhibit even larger dynamical mass-to-light ratios, these values are subject to numerous important caveats, as discussed later in this review.
} 
comprehensive historical excursus on the growing kinematic samples for MW dSphs).

The first attempt to go beyond the determination of a global l.o.s. velocity dispersion of a dSph was made by Mateo et al. (1991b) using a 2.5m telescope. These authors measured the kinematics of $\sim 30$ stars in the Fornax dSph, in the center and in a field located at about two core radii. This first l.o.s. velocity dispersion "profile" turned out to be approximately flat, and this led the authors to suggest that it could be due to a dark halo spatially more extended than the visible matter. These results opened a whole line of investigation to measure l.o.s. velocity dispersion profiles of dSphs around the Milky Way, and to use these to determine their dark matter (hereafter, DM) distribution, orbital structure and dynamical state.

The samples of l.o.s. velocities collected in the 90s contained a few dozens of individual members per dSph (e.g. Mateo et al., 1991b; Hargreaves et al., 1994a b; Olszewski et al., 1996; Queloz et al., 1995; Mateo et al., 1998). An increase in sample size became possible with multi-object spectrographs such as the KPNO/4m Hydra multi-fiber positioner (100 members in Draco and Ursa Minor, Armandroff et al., 1995), and the AF2/Wide Field Fibre Optical Spectrograph on the WHT (150 members in Draco, Kleyna et al., 2001).

In the second half of the 2000s several large spectroscopic surveys of the classical MW dSphs were carried out. In broad terms we can distinguish them in 3 main "streams":

1. Surveys that obtained l.o.s. velocities for typically $\sim 100-150$ members per dSph, with a large success ratio of dSph members/target stars thanks to an optimized target selection using Washington photometry (M, T2, and DDO51 filters, e.g. Majewski et al., 2005; Muñoz et al. 2005, 2006, Westfall et al., 2006; Sohn et al., 2007). These have made use of Keck/HIRES, Magellan/MIKE, CTIO/Hydra and Keck/DEIMOS.

2. Surveys to obtain several 100s of stars per dSph to determine both the internal kinematics and the metallicity distribution from Ca II triplet lines using intermediate resolution spectroscopy. This includes the Dwarf Abundances and Radial velocities Team (DART, PI: Tolstoy) ( 570, 800, 170 members for the Sculptor, Fornax, Sextans dSphs, respectively, at $R \sim 6500$ over the wavelength range $8200 \AA-9400 \AA$, Tolstoy et al., 2004, 2006; Battaglia et al., 2006; Helmi et al., 2006; Battaglia et al., 2008b; Starkenburg et al., 2010; Battaglia et al., 2011); 
and program 171.B-0520 (PI: Gilmore) "Towards the Temperature of Cold Dark Matter" ( 500, 170 members for the Carina and Leo II dSphs with the same set-up as for the DART data-set, Koch et al., 2006, 2007a). These have taken advantage of the VLT's large collecting area coupled to the wide-field, multi-object capability and stability of the FLAMES-GIRAFFE spectrograph (Pasquini et al., 2002) and, also of Keck/DEIMOS and GeminiN/GMOS (Koch et al., 2007c).

3. Surveys to obtain several 100 s to 1000 s of l.o.s. velocities and spectral indices (providing estimates of the relative metallicity of red giants) on a restricted wavelength range $(5140 \AA-5180 \AA)$ at resolution R 20000 (PI: M.Mateo, e.g. 800, 2500, 1400, 400 members for Carina, Fornax, Sculptor and Sextans, respectively Walker et al., 2007a, 2009a). These have been mainly carried out with the Michigan/MIKE Fiber System (MMFS) at the Magellan/Clay (6.5m) telescope and with MMT/Hectochelle (see Mateo et al., 2008, for Leo I). With a comparable field-of-view to FLAMES (20 arcmin), MMFS has the advantage of almost double the number of fibres (equally shared between the blue and red channel of the MIKE spectrograph).

Therefore, to-date the combined data-sets for the best studied dSphs have impressive sizes ( 2900 and 1700 probable members for Fornax and Sculptor, respectively), permitting studies of their internal properties to a level of detail that was unthinkable a little more than a decade ago.

The low luminosities of UFDs imply that very few RGB stars (the most luminous targets available for galaxies with old stellar populations) are available for spectroscopy. The size and spatial coverage of existing kinematic samples resemble those in the early days of the "classical" MW dSphs, even when targeting fainter stars (on the horizontal branch, and/or close to the main-sequence turn-off). Given our interest in exploiting the full l.o.s. velocity distribution, in what follows we concentrate on the "classical" dSphs and discuss only briefly results on the internal kinematics of UFDs.

\section{2. dSphs in a cosmological context}

In our current understanding of the Universe, a mere $5 \%$ of the total mass/energy density budget consists of baryons, atoms essentially, with the remaining $95 \%$ comprising about $24 \%$ non-baryonic "dark matter" and $71 \%$ "dark energy" (see Hinshaw et al., 2012, for the 9-years WMAP results). This has become known as the $\Lambda$ cold-dark matter $(\Lambda \mathrm{CDM})$ model. As the 
evocative naming suggests, we are ignorant of the nature of the great majority of constituents of the Universe.

There are several DM candidates such as weakly interacting massive particles, axions, sterile neutrinos, light gravitinos etc., whose existence is also motivated to solve problems in the Standard Model of particle physics (for a review see Feng, 2010). Some of these behave as cold and some as warm dark matter, where e.g. "cold" is defined as being non-relativistic at the time of structure formation. A wealth of experiments and strategies for direct and indirect detections of DM particles are underway (e.g. for reviews see Bertone et al., 2005; Hooper and Baltz, 2008; Feng, 2010), but at present the evidence for the existence of DM (based on the validity of Newton's law of gravity on all gravitational acceleration regimes) is provided by astrophysical observations on a variety of scales, from the smallest galaxies such as the dSphs up to the largest structures in the Universe $2^{2}$.

Potentially, astrophysical observations can provide important constraints on the dominant form of DM, as the characteristics of the DM particle are expected to influence the growth of structures, the substructure content and internal properties of DM halos. Rather than reviewing the extensive literature on the topic, we proceed to discuss results that are most directly related to this review, highlighting the crucial role of dwarf galaxies.

Cosmological pure DM N-body simulations, carried out in the $\Lambda$ CDM framework, show that the halos formed follow very specific functional forms, such as the Navarro, Frenk \& White profile (NFW, Navarro et al. 1996b, 1997)

$$
\rho(r)=\frac{\rho_{0}}{r / r_{s}\left(1+r / r_{s}\right)^{2}}
$$

where $\rho_{0}$ and $r_{s}$ are a characteristic density and radius. More recently the Einasto form has been found to provide better fits (e.g. Springel et al., 2008; Navarro et al., 2010)

$$
\rho(r)=\rho_{-2} \exp \left\{-\frac{2}{\alpha_{E}}\left[\left(\frac{r}{r_{-2}}\right)^{\alpha_{E}}-1\right]\right\},
$$

where $\rho_{-2}$ and $r_{-2}$ are the density and radius where the logarithmic slope

\footnotetext{
${ }^{2}$ Alternative theories of gravity, or modifications of Newton's law have also been presented in the literature. We decided not to discuss these here because their application to model the dynamics of dSphs has been very limited.
} 
$d \log \rho / d \log r=-\gamma_{D M}=-2$, and $\alpha_{E}$ is a shape parameter ${ }^{3}$. These density profiles are rather steep near the centre, with the NFW being cuspy with $\gamma_{D M}=1$, while the Einasto profile has $\gamma_{D M}=0$ at the centre.

Although not necessarily theoretically motivated, other density profiles are also often employed in the literature. Typically they have the form

$$
\rho(r)=\frac{\rho_{0}}{\left(r / r_{s}\right)^{\gamma}\left(1+\left(r / r_{s}\right)^{\kappa}\right)^{(\alpha-\gamma) / \kappa}},
$$

where $\alpha, \gamma, \kappa \geq 0$. Note that $\gamma$ and $\alpha$ correspond to the inner and outer slopes respectively. The sharpness of the transition between these two regimes is thus given by $\kappa$. A cuspy profile has $\gamma>0$, while for a cored one $\gamma=0$ and $\kappa>1$. This is because in the cored case, the profile must have a flat shape at the centre, i.e. $d \rho / d r=0$. A profile that has $\gamma=0$ and $\kappa \leq 1$ has at the centre $d \log \rho / d \log r=0$ and a finite density, but in this case $d \rho / d r$ is non-zero, and hence this profile should not be confused with a core.

In the $\Lambda$ CDM high-resolution cosmological N-body simulations described above the sub-halo mass function of MW-sized main halos is $d N / d M \propto$ $M^{-\alpha}$, with $\alpha=1.9$ down to the simulations' resolution limit (Springel et al., 2008), which is smaller than the mass estimates for the faintest dSphs (see Sec. 3). These simulations predict that MW halos contain $20 \%$ of the mass in subhalos, which results in a very large number of (mostly extremely low mass) satellites.

A comparison between the results of these pure DM N-body simulations with observations on galactic scales is not straightforward. Part of the issue lies in making the link between a luminous satellite to what should be its corresponding sub-halo in a DM simulation (e.g. of what mass? how dense?, see Strigari et al., 2010). This is particularly difficult because such simulations do not include baryons. This has motivated numerous theoretical efforts to provide a realistic treatment of baryonic effects using semi-analytical models and hydrodynamical simulations of dwarf galaxies (e.g. Revaz et al., 2009; Li et al., 2010; Font et al., 2011; Sawala et al., 2012; Starkenburg et al., 2013). Observationally, it is clearly important to obtain reliable estimates of the mass content and its distribution in dwarf galaxies.

For example, there is a debate about the inner shape of the density profiles of the DM halos hosting galaxies. For dSphs, this issue is still very

\footnotetext{
${ }^{3}$ For $\alpha_{E} \sim 0.2$ the resulting profile resembles an NFW.
} 
open (see Sec. 3). On the other hand, for isolated late-type dwarfs and low surface brightness galaxies, the rotation curves seem to favor cored rather than cusped DM distributions (e.g. de Blok, 2010, and references therein). It has been suggested that feedback from supernovae explosions for these more massive systems could transform a cuspy halo into a cored one (e.g. Navarro et al., 1996a; Read and Gilmore, 2005; Governato et al., 2010; Pontzen and Governato, 2012; Teyssier et al., 2013). Note that in the case of an UFD, a single SN event releases an amount of energy comparable to the binding energy of the whole system. On the other hand, it is still to be assessed whether this mechanism is important or relevant on the scales of the MW dSphs, also given their low star formation rates (see Peñarrubia et al., 2012).

The "missing satellites" problem refers to the large mismatch between the observed number of dwarf galaxies satellites of the MW and M31 and the predicted number of DM subhalos (Klypin et al., 1999; Moore et al. 1999). The discovery of dozens low-luminosity dwarf galaxies in the Local Group, mainly by SDSS around the MW (e.g. Willman et al., 2005; Zucker et al., 2006; Belokurov et al., 2006, 2007, to mention a few) and the PandAS survey for M31 (e.g. McConnachie et al., 2009; Martin et al., 2009), has mitigated somewhat the "missing satellite" problem, after taking into account the surveys' coverage and selection function (Koposov et al., 2009). The most appealing solution to reconcile predictions and observations is to suppress star formation, or gas accretion, in low-mass halos because of the joint effects of feedback and of a photo-ionizing background due to re-ionization (e.g. Bullock et al., 2000; Benson et al., 2002; Somerville, 2002).

Another interesting issue was the recently reported "too big too fail problem" pointed out by Boylan-Kolchin et al. (2011), who used the Aquarius suite of DM simulations to argue that there exists a population of subhalos that are too massive and too dense to be consistent with the internal kinematics of the MW dSphs, and yet they do not have an observed stellar counterpart. However, as argued by Wang et al. (2012) and Vera-Ciro et al. (2012), the number of massive satellites is a stochastic quantity that also depends on the mass of the host. For example, if the mass of the MW is around $8 \times 10^{11} \mathrm{M}_{\odot}$, i.e. the least massive MW-like halos of the Aquarius suite (which reproduces well the observed MW satellite luminosity function, see Koposov et al., 2008; Starkenburg et al., 2013), the mismatch disappears. Furthermore, Vera-Ciro et al. (2012) show that M31, if assumed to be more massive than the Milky Way, does not miss such a population.

A plausible alternative to CDM is warm dark matter (WDM). The warm 
component has the effect of reducing the small-scale power in the primordial fluctuations spectrum, yielding fewer subhalos and of lower central densities (Colín et al., 2000, 2008; Lovell et al., 2012). Specifically, in the numerical simulations of Macciò et al. $(2012,2013)$, which explore a range of masses for the WDM particles, cored density profiles arise naturally. However, either the core sizes are too small to be consistent with those suggested in some studies of the internal kinematics of MW dSphs (see Sec. 3) or if large enough, they would be due to particles whose masses are inconsistent with the limits imposed by observations of the Lyman- $\alpha$ forest (e.g. Viel et al., 2005; Seljak et al., 2006; Viel et al., 2008). Note however that e.g. Busha et al. (2007) find in their WDM simulations that the halos are well described by an NFW form (i.e. cuspy) while Wang and White (2009) find this even holds for halos in hot dark matter simulations. Given that the state-of-the-art of WDM simulations is not as extensive and developed as for CDM, we await future developments.

From the above it is clear that there are numerous reasons to try and pin down the DM content and its distribution in the dSphs. Given that the overall evolution of small systems like dwarf galaxies will most likely be sensitive to their relatively small potential well (e.g. Revaz and Jablonka, 2012; Sawala et al., 2012), obtaining such measurements will also allow us to make sense of the variety of star formation and chemical enrichment histories of these galaxies, in particular in conjunction with the information on the dSphs orbital history that the Gaia satellite mission (Prusti, 2011) will provide.

\subsection{Are the stellar components of dwarf spheroidals affected by tides or are they in dynamical equilibrium?}

An assumption in dynamical modeling of dSphs is that these objects are in dynamical equilibrium, while if they were significantly affected by tidal interactions with the MW this would need to be taken into account.

The possibility that dSphs are fully tidally disrupted dark-matter free galaxies has been excluded on the basis of their observed internal kinematic and structural properties (see for example Klessen et al., 2003; Muñoz et al., 2008), the large distances of some of these galaxies (up to $250 \mathrm{kpc}$ from the MW) and a well-established luminosity-metallicity relation. It would also be difficult to explain the dSphs' extended SFHs and broad metallicity distributions (see e.g. Tolstoy et al., 2004; Battaglia et al., 2006; Koch et al., 2006; Starkenburg et al., 2010; Battaglia et al., 2011) if the potential well 
would be due solely to the dSph stars (amounting to typically $10^{5}-10^{6} \mathrm{M}_{\odot}$, e.g. McConnachie 2012).

Partly because of the lack of knowledge of the orbits of dSphs around the MW, the importance of tides on the stellar components of dSphs is largely unknown. This also depends on the degree of embedding of this component in its dark matter halo, as well as on the average density of the system. Mayer et al. (2001) propose that $\mathrm{dSph}$ galaxies are what results when a disky dwarf is tidally stirred by the MW. For this process to be effective, the stellar component of the dSph today has to be tidally limited, in which case tidal tails are expected. However, Peñarrubia et al. (2008b) find that the stars are very resilient to tides in their simulations where the stellar component follows a King-profile and is deeply embedded in an NFW halo. In any case, there is general consensus that the central velocity dispersion (or the dispersion at the half-light radius) continues being a good indicator of the present maximum circular velocity and bound mass, as long as the objects retain a bound core (e.g. Muñoz et al., 2008; Peñarrubia et al., 2008b; Klimentowski et al., 2009; Kazantzidis et al., 2011a).

Besides the obvious case of Sagittarius, the only classical dSph presenting unambiguous signs of tidal disturbance such as tails and isophote twists is Carina (Battaglia et al., 2012). This object has been a candidate for tidal disturbance for a long time, with convincing arguments given by the presence of spectroscopically confirmed RGB stars, probable members, out to very large distances from its center (4.5 times the central King limiting radius), observed together with a break in the surface brightness profile, a velocity shear with turn-around, and a rising line-of-sight velocity dispersion profile (e.g. Muñoz et al. 2006). Among the classical dSphs, other candidates for tidal disruption are Leo I (e.g. Sohn et al., 2007; Mateo et al., 2008) and Ursa Minor (e.g. Martínez-Delgado et al., 2001; Palma et al., 2003; Muñoz et al., 2005), although the observational evidence is not as strong as for Carina. Note that even for Carina, the N-body simulations by Muñoz et al. (2008) show that large amounts of $\mathrm{DM}\left(M / L \sim 40 \mathrm{M}_{\odot} / \mathrm{L}_{\odot}\right)$ within the remaining bound core are still needed to explain its characteristics.

N-body simulations of tidally perturbed dSphs agree in predicting rising l.o.s. velocity dispersion profiles in the majority of cases, while only Carina and perhaps Draco (Walker, 2012) are observed to show such feature. Together with the fact that most classical dSphs show no tidal streams, this may be taken as indicative that the outer parts of the stellar components of dSphs have not been significantly affected by tides. All these arguments 
provide some justification for the assumptions made in this review, namely that we may consider the dSphs to be in dynamical equilibrium.

However, it would be well-worth the effort to carry out observational campaigns designed to maximize the chances of detecting the smoking-gun signature of tidal disruption, i.e. tidal tails. Detection of these low-surface brightness features needs deep and spatially extended photometric data-sets. Instruments like CTIO/DECam and the forthcoming Subaru/HyperCam, but also the proper motion information from the Gaia mission, are excellent matches to this type of problem.

\section{Observed kinematics}

The heliocentric distances to MW dSphs, ranging from 75 to $250 \mathrm{kpc}$, have made it unfeasible to obtain accurate proper motions of individual stars in these galaxies with current facilities. This implies that we only have access to one component of their velocity vector, namely that along line-of-sight (l.o.s.) $v_{\text {l.o.s }}$. Therefore, all current studies of the internal kinematics of dwarf galaxies are based on their line-of-sight velocity distributions (LOSVD) and their moments.

In this Section we start by describing how to derive a reliable LOSVD from the measurements of the velocities of individual stars. In Sec. 2.1 we consider the effects of velocity errors, the presence of binary stars and the contamination introduced by objects that do not belong to the dSph, such as Milky Way stars (hereafter "contaminants" or "interlopers"). In Sec. 2.2 we discuss how the main characteristics of an LOSVD are related to the internal dynamics of the system. In Sec. 2.3 we describe how to measure the moments of the LOSVD, and present the latest results for the MW dSphs.

\subsection{Derivation of a reliable LOSVD}

\subsubsection{Velocity errors}

In general l.o.s. velocities accurate to a few $\mathrm{km} \mathrm{s}^{-1}$ are essential to kinematic studies of dSphs, given their low internal velocity dispersion and small velocity gradients (see Sec. 2.3).

Koposov et al. (2011) have shown that it is crucial to obtain a reliable estimation of the velocity errors to provide a realistic value of the dSph

$\sigma_{\text {l.o.s }}$, and hence of its dynamical mass (see Sec. 3). Fig. 1 shows that if the velocity errors are perfectly known, the intrinsic (true) $\sigma_{\text {l.o.s }}$ can be accurately recovered even if the measurement errors are larger than the intrinsic $\sigma_{\text {l.o.s }}$. 
On the other hand, overestimated (pessimistic) errors will yield a smaller estimate for the dispersion, while the opposite happens for underestimated errors. This effect becomes particularly strong when the velocity error is $\gtrsim 0.5$ the true $\sigma_{\text {l.o.s }}$.

In the case of the classical dSphs, with $\sigma_{\text {l.o.s }} \sim 6-11 \mathrm{~km} \mathrm{~s}^{-1}$, this is not an issue. With current fiber-feb spectrographs, it is possible to obtain very accurate $v_{\text {l.o.s }}$ for their red giant branch stars. For example, with 1 h observing time at VLT/FLAMES+GIRAFFE at intermediate resolution ( $\mathrm{R} \sim 6500)$ one can achieve velocity errors to better than $\sim 5 \mathrm{~km} \mathrm{~s}^{-1}$ on RGB stars with $\mathrm{V}=19.5$ in the region of the nIR Ca II triplet lines (e.g. Battaglia et al., 2008b). This corresponds to $\sim 0.5 \mathrm{mag}$ below the tip of the RGB of the most distant MW dSph, Leo I. The RGB of MW classical dSphs is well-populated, making it unnecessary to observe fainter stars to gather a large sample of targets. The situation is different for the UFDs, both because of the need to target fainter stars but also because of their intrinsically smaller $\sigma_{\text {l.o.s }}$.

An example of the importance of well-determined velocity errors is given by Koposov et al. (2011), who developed a sophisticated data-reduction procedure for VLT/FLAMES+GIRAFFE data. In their application to the Boötes I UFD, and after the removal of radial velocity variable stars, this allowed Koposov et al. (2011) to "resolve" the LOSVD into two Gaussians with very different velocity dispersions. As highlighted by the authors, this result shows that current determination of masses for UFDs need to be taken with care (Martin et al., 2007; Wolf et al., 2010; Koposov et al., 2011).

\subsubsection{Binaries}

In this review, we are mostly concerned with the effect that binaries may have on the LOSVD and its moments, for example by inflating the measured velocity dispersion of dSphs. This concern is as old as the first measurements of a dynamical mass-to-light ratio of these objects (Olszewski et al., 1996). Hargreaves et al. (1996) concluded that the highest velocity dispersion that could stem from the orbital motions of binaries alone is 3 $\mathrm{km} \mathrm{s}^{-1}$ if the binary population is similar to that of the MW. The authors state "To produce larger dispersions, more binary orbits with a mixture of lower periods, higher mass secondaries or primaries with radii smaller than $10 R_{\odot}$ are required". More recently, Minor et al. (2010) showed that, if the measured velocity dispersion of a dSph ranges between 4 and $10 \mathrm{~km} \mathrm{~s}^{-1}$, the inflation from binaries should not be more than $20 \%$ in samples of RGB stars with absolute magnitude $\mathrm{M}_{V} \lesssim 1$ and older than 1 Gyr (again assuming 
similar binary populations as in the MW), and at most $30 \%$ when including fainter stars. The authors also devise a method to correct the measured velocity dispersion for the contribution of binaries that is expected to yield intrinsic velocity dispersions accurate to a few percent. They do this by measuring the fraction of stars that exhibit a change in velocity exceeding a certain threshold over a time between measurements.

Therefore, the general conclusion is that the effect of binaries for the $\sigma_{\text {l.o.s }}$ measured for dSphs is minor. Unidentified binaries can instead have a strong impact on the $\sigma_{\text {l.o.s }}$ measured for UFDs. McConnachie and Côté (2010) explore whether it is plausible to explain the velocity dispersions of UFDs with $\mathrm{M}_{V} \gtrsim-7$ as the result of inflation by unidentified binaries from an intrinsic dispersion of $\sim 0.1-0.3 \mathrm{~km} \mathrm{~s}^{-1}$ (i.e. they test whether UFDs could be devoid of DM). The authors consider only binaries with periods smaller than 10-100 yr (for comparison, the mean period of binaries in the solar neighborhood is $180 \mathrm{yr}$, Duquennoy and Mayor, 1991). McConnachie and Côté (2010) find that binaries cannot account for observed dispersions much in excess of $\sim 4.5 \mathrm{~km} \mathrm{~s}^{-1}$, but contamination by binaries might still be a concern for some UFDs.

In their analysis of the Segue 1 UFD, Martinez et al. (2011) introduce a comprehensive Bayesian method to analyze multi-epoch data, including foreground contamination from the MW and the presence of binaries. Their analysis shows that, if in dynamical equilibrium, then Segue 1 has an intrinsic velocity dispersion of $3.7_{-1.1}^{+1.4} \mathrm{~km} \mathrm{~s}^{-1}$ at $1 \sigma$. They estimate that without a binary correction, the most likely dispersion would only have been $12 \%$ larger. However, the authors point out the importance of multi-epoch data to disfavor the possibility that most of Segue 1 observed dispersion is due to binary stars.

The determination of the binary fraction and distribution of orbital parameters in dSphs is also of intrinsic interest to establish dependences on different galactic environments (for a nice introduction see Minor, 2013), but no such dedicated campaigns have been carried out to-date. The recent work of Minor (2013) constitutes the best effort in determining the binary fraction in MW dSphs by exploiting the information on repeated observations from the large spectroscopic survey of Walker et al. (2009a). The binary fraction in $3 \mathrm{dSphs}$ is found to be consistent with the one of the MW (around 0.5), while it appears to be much lower for Carina (Car). Unfortunately, the binary fraction and period distribution parameters are strongly degenerate: a small binary fraction with short mean period produces very similar observed 
velocity variations (within the measurement errors) to a large binary fraction with long mean periods.

To constrain the period distribution independently from the binary fraction, the measurement errors on the individual velocities need to be $<0.1$ $\mathrm{km} \mathrm{s}^{-1}$ (achievable with high resolution spectrographs, see Eq. (19) of Minor, 2013), unless the measurements are taken over time-scales much longer than 5 years. This is therefore a difficult task, and larger amplitude velocity variations would only be measurable if the mean period in dSphs is considerably shorter than for MW field stars.

\subsubsection{Weeding out Galactic contaminants}

Contamination by MW stars on the LOSVD needs to be modeled carefully. An example is the case of Willman 1, whose nature as a galaxy or stellar cluster (as inferred by the presence/absence of an $[\mathrm{Fe} / \mathrm{H}]$ spread among its stars) is still debated due to the difficulty of gathering interloper-free samples of members (Martin et al., 2007; Siegel et al., 2008; Willman et al., 2011).

Methods for removal of contaminants have different levels of sophistication and effectiveness, and make use of different sets of observables, such as the magnitude and colors of the objects, their velocities and their spectral features. We describe these in detail in what follows, and leave for Sec. 2.3.3 how to model the LOSVD including a foreground component.

The photometric data provide the first aid in minimizing the presence of interlopers: resolved galaxies can be weeded out using information on shape parameters (unresolved galaxies usually are not an issue at these magnitudes); while a fraction of MW stars can be excluded by restricting target selection to a range of magnitude and colors consistent with the locus of specific stellar evolutionary phases at the distance of the dwarf galaxy (e.g. the RGB, main sequence turn-off, horizontal branch etc). Even then, the large solid angles subtended by MW dwarf galaxies can result in severe contamination from MW stars, depending on the Galactic coordinates of the object. This is the case for example, of Sextans (Sext), as shown in Figure 2(top-left panel), whose RGB is barely visible, being buried by MW stars. Fortunately, spectroscopic observations come to our aid.

Information from the stars kinematics. Assuming that the velocities of the stars belonging to the dwarf follow a Gaussian distribution, the simplest approach to remove MW contaminants is to adopt a hard cut in $v_{\text {l.o.s. }}$ by iteratively clipping the sample at a given number of $\sigma_{\text {l.o.s }}$ from the systemic 
velocity $v_{\text {sys }}$ (where $\sigma_{\text {l.o.s }}$ is an estimate of the width of the LOSVD, such as the standard deviation or robust bi-weight estimator, e.g. Mateo et al., 1991a; Walker et al., 2006). Although in general the outer parts need to be treated more carefully, this method may suffice if there is not much overlap between the LOSVD of the MW and of the dSph, otherwise more sophisticated methods are to be preferred. Such methods simultaneously model the expected LOSVD of MW and dSph stars, and take into account that the probability that a star is a member of the dSph decreases as a function of projected radius $R$. This follows from the fact that the projected number density of dSph stars decreases with $R$, while the projected number density of MW stars is roughly constant over the area subtended by the dwarf (see Sec. 2.3.3).

Diagnostics sensitive to gravity from spectroscopy. Since the great majority of spectroscopic studies on MW dSphs target RGB stars, and most contaminants are dwarf stars in the Galactic disk(s), diagnostics sensitive to surface gravity can strengthen membership determination.

A popular indicator is the equivalent width $(\mathrm{EW})$ of the $\mathrm{Na}$ I doublet lines at $\lambda=8183,8195 \AA$, which Schiavon et al. (1997) showed is a useful dwarf/giant stars discriminator as dwarf stars exhibit larger $\mathrm{Na} I$ EWs than giant stars. As shown in Fig. 2 (top-right panel), the Na I EW of dwarf stars declines with increasing temperature: at $T_{\text {eff }}<4000 \mathrm{~K}$ there is a very marked difference in Na I EW between dwarf and giant stars, making the discriminator very powerful, while at $T_{\text {eff }}>4000 \mathrm{~K}$ the $\mathrm{Na}$ I EW of dwarf stars almost approaches the values of giants (as shown in the topright panel of Fig. 2). Although some studies have used hard-cuts on the Na I EW to separate dwarfs from giants (e.g. for UFDs, Martin et al., 2007; Simon and Geha, 2007), other works have considered the location of the stars in the $\mathrm{Na}$ I EW vs de-reddened $(\mathrm{V}-\mathrm{I})_{0}$ color plane, to take into account the aforementioned dependence of the Na I EW of dwarf stars on $T_{\text {eff }}$ (e.g. Gilbert et al., 2006; Guhathakurta et al., 2006; Geha et al., 2010, for applications to the M31 system using training sets).

Walker et al. (2007a) introduced the use of the Mg I index to assign membership of stars to their samples of MW dSphs. This is similar in spirit to the reduced CaT EW (e.g. Martinez et al., 2011) or equivalently the $[\mathrm{Fe} / \mathrm{H}]$ values derived from CaT calibration (e.g. Helmi et al., 2006). There is some degree of overlap between the distribution of $\mathrm{Mg}$ I index or $\mathrm{CaT}[\mathrm{Fe} / \mathrm{H}]$ of MW and dSph stars (see e.g. Figs. 1 and 2 of Walker et al., 2009c, and Fig. 1 
of Helmi et al. 2006). This also happens for the EW of the nIR MgIline at $\lambda \sim 8807 \AA$, as shown by Battaglia and Starkenburg (2012) (see their Fig. 7). Battaglia and Starkenburg (2012) have suggested that the position of a star in the MgIEW versus CaT EW plane, rather than just its $\mathrm{MgIEW}$, is a much more efficient dwarfs/giants discriminator over the range $-2 \leq[\mathrm{Fe} / \mathrm{H}]$ $\leq 0$ (see bottom panel in Fig. 2). The relation to discriminate giants and dwarfs by Battaglia and Starkenburg (2012) is:

$$
\mathrm{EW}_{\mathrm{Mg}}(m \AA)= \begin{cases}300 & \text { if } \lambda \leq 3750 \mathrm{~m} \AA \\ 0.26 \times \Sigma \mathrm{W}_{\mathrm{CaT}}-670.6 & \text { if } \lambda>3750 \mathrm{~m} \AA\end{cases}
$$

where $\Sigma \mathrm{W}_{\mathrm{CaT}}$ is the summed EW of the two strongest CaT lines.

Other diagnostics such as the EWs of the K7665 and K7699 absorption features and the strengths of the $\mathrm{TiO}$ bands at 7100,7600 , and $8500 \AA$, are effective at dereddened $(\mathrm{V}-\mathrm{I})_{0}>2.5$ (Gilbert et al., 2006).

Diagnostics sensitive to gravity from photometry. Photometric indices sensitive to gravity (and luminosity, effective temperature, etc.) can be derived from combinations of particular filters. For example, the intermediate-band DDO 51 filter measures the strength of the $\mathrm{Mg} \mathrm{b} / \mathrm{MgH}$ feature near $5170 \AA$, which is strong in late-type dwarfs and weak in giants (e.g. Morrison et al., 2001), and is in general used in combination to the Washington $M$ and $T_{2}$ filters (e.g. Majewski et al., 2000; Palma et al., 2003). However, verification of membership with velocity information is crucial if the area considered is large enough to contain a significant number of metal-poor halo dwarfs which cannot be isolated with this method either at these faint magnitudes and/or if low photometric precision moves stars from the dwarfs to the giants locus in the $\mathrm{M}-\mathrm{T}_{2}$ vs $\mathrm{M}-51$ plane (e.g. Morrison et al., 2001). This method allowed Muñoz et al. (2005) to find members of the Car dSph out to very large distances from its center. Strömgren photometry (filters $u, v, b$ and $y$, where the first two and partially $b$ are covered by the broader Washington Cfilter) has been successfully used to determine membership in the Draco dSph (Faria et al., 2007), and to weed out non-members in the sparsely populated Hercules, leading to a decrease of its estimated dynamical mass (Adén et al. 2009a).

Choosing what membership method to adopt probably implies a trade-off between available facilities, observing times involved, permitted tolerance to the presence of contaminants, and expected stellar population mix both in 
the target galaxy and foreground population along the specific line-of-sight. It is important to keep in mind that, even if the inclusion of the kinematic information often is crucial in distinguishing contaminants from member stars, the methods that use such information need to make assumptions on the intrinsic LOSVD of the dwarf, which is instead what we want to determine. It may be worth to assess the effectiveness of cleaning up samples only on the basis of diagnostics independent of kinematics or when adopting less strict kinematic criteria; for example, Battaglia and Starkenburg (2012) show that the Mg I line method is still very effective when relaxing the kinematic criterion from the more standard $3 \sigma$ clipping to a larger $4 \sigma$, which would include the high velocity tails of the dSph LOSVD.

The situation will improve dramatically for MW dSphs when parallax and proper motion information becomes available from the Gaia mission. Most foreground dwarf stars will be identified thanks to their large parallaxes and proper motions, while even metal-poor MW halo giants (nowadays the most difficult contaminants because they have similar spectral features as RGB stars in the dwarf galaxy), could be identified through their different tangential velocities, and possibly also their parallaxes. In either case, it will be important to develop models that include all populations of contaminants, as suggested by Walker et al. (2009c).

\subsection{Characterization of the line-of-sight velocity distribution}

The shape of the LOSVD depends both on the distribution function (DF) of the stars as well as on the gravitational potential in which these orbit. Here we focus specifically on spherical systems and present some general results on what can be learned from the characteristics of the LOSVD. There is extensive literature on the topic from the dynamical modeling of elliptical galaxies, but we choose here to briefly review a few results that may be particularly useful for dSphs. It is important to stress that there are two important differences between elliptical galaxies and dSphs. Firstly, baryons are not negligible in ellipticals. Secondly, in contrast to the distant ellipticals, in principle, the individual kinematic measurements for the stars in a dSph could be used to characterize the LOSVD, however most works use its moments, as we shall see in Sec. 3 .

Dejonghe (1987) has studied the LOSVDs and its moments for selfconsistent Plummer anisotropic models. He has shown that in the central

regions the $\sigma_{\text {l.o.s }}$ has a stronger contribution of radial orbits even if the system 
is tangentially anisotropic. This is known as the "complementarity" property, and is simply the result of projection effects. Moreover, a more peaked LOSVD results for a tangentially biased system than for a radial one when looking in the central regions; however, a tangentially anisotropic ellipsoid can be recognized in the outer parts, as a flat-topped LOSVD.

Gerhard (1993) has explored the shape of the LOSVD in the case of different gravitational potentials (Kepler and the isothermal sphere), for power-law light distributions for the tracers, and for a family of DFs that are quasiseparable in energy and total angular momentum. Note that this study is particularly relevant for dSphs since it also deals with non self-consistent systems. Gerhard (1991, 1993) show that the velocity profiles (VPs) ${ }^{4}$ for self-consistent radially anisotropic models are strongly non-Gaussian (the isotropic case is Gaussian), with a two component structure: a narrow inner core and strong extended outer wings. As the stellar density profile steepens, the VP becomes more dominated by the inner core and the relative amplitude of the extended wings with respect to the core decreases, as shown in Fig. 3. When the system is embedded in an isothermal potential, tangentially anisotropic models produce nearly Gaussian VPs with flattened tops. These become more noticeable for steeply falling stellar density profiles. In the Keplerian potential instead, the VP in the isotropic case is not Gaussian, and it becomes more Gaussian-like for radially anisotropic systems. Nonetheless also here, the VPs in the tangential case are flat-topped. Overall, strongly tangentially anisotropic DFs in the outer parts lead to flat-topped VPs that are easily recognizable, and this effect does not depend strongly on the potential, but it becomes more enhanced for steeper stellar density profiles, as shown in Fig. 3. This result is also supported by the work of Wilkinson et al. (2002) who have introduced a family of constant anisotropy models where the stars follow a Plummer sphere, while for the DM halo they discuss the mass-follows-light case, an isothermal sphere and an extended halo with a harmonic core. Wilkinson et al. (2002) reach a similar conclusion, namely that it is the VP in the outer parts that holds most information on the velocity anisotropy.

The results above indicate that shape of the LOSVD, mainly how peaked or flat-topped it is especially at large radii, could be directly be used to infer the orbital structure of a dSph in this regime. This shape information

\footnotetext{
${ }^{4}$ Here we use the terms velocity profiles VPs and LOSVD, interchangeably.
} 
is encoded in its 4th moment, be it the kurtosis or the Gauss-Hermite $h_{4}$ moment, see Sec. 2.3.4. In the outskirts of a dSph, its stellar density profile may be assumed to fall off as a power-law, hence in this region the conclusions drawn by Gerhard (1993) would be most relevant. In that case, the LOSVDs should be more flat-topped for a tangentially anisotropic ellipsoid. Furthermore, this result also ought to be valid for systems with multiple stellar components, as this outer region is generally dominated by a single (the most metal-poor) component (see Sect. 2.3.5).

\subsection{Results}

In this section, we discuss step by the step how to measure the first four moments of the LOSVD for dSphs, as well as present some first results.

\subsubsection{Surface brightness profiles}

Even though not a moment of the LOSVD, we briefly describe the dSphs surface brightness profile (as derived from stellar number counts) as this is an ingredient for the dynamical modeling.

The results from the literature are rather heterogeneous as the surface stellar number count profiles are derived from samples of different photometric depths/completeness, spatial extent, and available regions for accurate determination of the (foreground) density. Studies in the literature have mostly focused on the overall fit at all radii and in the performance of various functional forms in the outer parts as a possible way of detecting signs of tidal stripping from the MW.

Typically, empirical King models (King, 1962) fit better the MW dSphs surface stellar number counts profile with respect to exponential profiles (Irwin and Hatzidimitriou, 1995). However, it is now well-established that King models are not necessarily good representations of the outer parts, as they typically underestimate the observed densities (e.g. Irwin and Hatzidimitriou, 1995: Martínez-Delgado et al., 2001; Wilkinson et al., 2004; Coleman et al., 2005; Battaglia et al., 2006, 2008a). Note that, if the stellar component of MW dSphs is embedded in massive and extended DM halos, such an excess does not need to be interpreted as evidence for tidal disruption.

Although not tested for all MW dSphs, in some cases better representations at all radii are given by Sersic profiles (Sersic, 1968) that decline faster than exponentials (e.g. Odenkirchen et al., 2001; Battaglia et al., 2006) or by Plummer profiles (e.g. Battaglia et al., 2008a). Fits to the light distribution in the very central regions have not received much attention thus far. 
The variety of profiles used allow for the presence of cores as well as small (logarithmic) cusps in the intrinsic 3D luminosity density profile.

\subsubsection{The first moment: velocity gradients}

A variation of the mean (or median) l.o.s. velocity across a dSph (hereafter "velocity gradients") potentially allows us to gain information on the physical mechanisms shaping the dSphs, such as intrinsic rotation or tidal disruption. However, velocity gradients also result from mere geometrical effects due to the perspective orbital motion of the dwarf galaxy around the Milky Way ${ }^{5}$ and we refer to these as "spurious" gradients.

A variety of methods have been used in the past to measure the variation of the LOSVD first moment across the object: $i$ ) considering how the average/median velocity (in the heliocentric or Galactocentric Standard of Rest [GSR] frame) varies as a function of distance along some direction (e.g. Muñoz et al., 2006); $i$ ) as before, but considering stars falling within "slits" of varying position angle (PA, e.g. Battaglia et al., 2008a); iii) calculating the mean velocity differences, $\Delta v$, on either side of a bisector line passing through the galaxy center and having a range of position angles, $\theta$ (see Walker et al., 2006; Mateo et al., 2008, e.g.); $i v$ ) exploring the variation of the mean velocity in angular sectors (Amorisco and Evans, 2012c).

Until a few years ago, no statistically significant velocity gradients had been detected from spectroscopic samples limited to the central regions of dSphs, except for a $3 \sigma$ detection along the minor axis of Ursa Minor but from a sample of only 35 stars (Hargreaves et al. 1994). In the past decade this has changed considerably thanks to the large increase in the size and spatial coverage of kinematic samples of MW dSphs brought by wide-area multiobject spectrographs. At present, statistically significant velocity gradients have been reported for Sculptor (Scl), Car, Fornax (Fnx), Sext and Leo I (Muñoz et al., 2006; Battaglia et al., 2008a; Mateo et al., 2008; Walker et al., 2008; Battaglia et al., 2011). The weak velocity gradient along the projected major axis of Leo II is significant only at the $0.17 \sigma$ level (Koch et al., 2007a), and Sext could certainly benefit from a larger spatial coverage. The velocity gradients measured are of only a few $\mathrm{km} \mathrm{s}^{-1} \mathrm{deg}^{-1}$.

\footnotetext{
${ }^{5}$ The mean velocity of a star in an object with a large angular extent such as MW dSphs is expected to vary as a function of position of the star because of geometrical projection effects due the proper motion of the object (e.g. Feast, Thackeray \& Wesselink 1961). Such "spurious" velocity gradients mimic solid body rotation.
} 
The importance of exploring the outer regions of dSphs to detect such gradients has become increasingly evident. For some dSphs the mean velocity is found to slowly rise ${ }^{6}$ with projected radius $R$ (see Fig. 1 of Battaglia et al., $2008 \mathrm{a}$, for the case of Scl). It is then in the outer regions that the mean and systemic velocities will likely differ the most, while at the center the system is basically entirely dominated by velocity dispersion. The case of Leo I, where the presence of a gradient was investigated via the bisector method, illustrates this clearly as shown in Fig. 4: no signs of a velocity gradient are visible at $R<400$ ", while the detection becomes statistically significant when considering stars at projected radii $>600 "$. On the other hand, application of the bisector method to the whole sample, i.e. not including the projected distance information, yields no compelling evidence for a significant velocity gradient, indicating that, at least in this case, such method washes out the larger signal present in the outer parts. Another, although indirect, example is given by Car, for which Muñoz et al. (2006) detect a clear velocity gradient with a velocity amplitude of $\sim 5 \mathrm{~km} \mathrm{~s}^{-1}$ over a scale of $1.2 \mathrm{deg}$, while a much lower value is found by Walker et al. (2008) for a sample restricted to a smaller spatial region. Homogeneous spatial coverage is also essential to recover the direction of the strongest gradient.

The origin of the detected velocity gradients could in principle impact the mass modeling. If the detected velocity gradients are entirely due to perspective effects ("spurious"), the only action required would be to place the velocities in a system at rest with the dwarf galaxy. However, for ratios of maximum apparent versus intrinsic velocity dispersion $v / \sigma \sim 0.5-0.6$, as observed in dSphs, Amorisco and Evans (2012c) show that it may not even be necessary to subtract such velocity gradients as the impact is merely an increase of $10 \%$ in the observed dispersion compared to the intrinsic one, while the change in the higher moments is less than what can be detected with sample size as large as 800 stars.

Although desirable, the correction for the 3D motion of the dwarf galaxy around the MW is made uncertain by the large errors on the current measurements of their proper motion (in reality, for some dSphs entirely lack astrometric proper motion measurements). This is illustrated in Fig. 5, where we see how the amplitude and direction of the "spurious" gradients may vary

\footnotetext{
${ }^{6}$ From current samples it appears difficult to pin down the exact shape of the velocity gradient, whether it can be approximated by a straight-line or other functional forms.
} 
according to the systemic proper motion measurements existing in the literature, taking the Scl dSph as an example. Here we have randomly generated a uniform distribution of stars in right ascension and declination, with no intrinsic velocity assigned. As shown in the top left panel of this figure, when adopting Piatek et al. (2006) proper motion the amplitude (at the last measured point) is very small, less than $1 \mathrm{~km} \mathrm{~s}^{-1}$ and the gradient points at PA $\sim 75 \mathrm{deg}$. However it is enough to consider $\mu_{\alpha}-1 \sigma$ and $\mu_{\delta}+1 \sigma$, as shown in the top right panel, to make the gradient direction change considerably, with the vector now pointing at P.A. $\sim-15 \mathrm{deg}$ (the amplitude is still $\sim 1 \mathrm{~km} \mathrm{~s}^{-1}$ ). On the other hand, the amplitude obtained when adopting the proper motion from Schweitzer et al. (1995) is larger, up to $4 \mathrm{~km} \mathrm{~s}^{-1}$ (bottom left). We note that the "spurious" solid-body rotation results in velocity fields with parallel iso-velocity contours, perpendicular to the proper motion direction; however, the intrinsic velocity dispersion of dSphs makes it challenging to detect such a signature with current data-sets.

It is also possible to assume that $\mathrm{dSphs}$ do not have intrinsic velocity gradients, and derive a systemic proper motion from the measured stellar redshifts (Walker et al., 2008). For Scl, there is disagreement with the direct proper motion measurements by Schweitzer et al. (1995); Piatek et al. (2006) and those derived by Walker et al. (2008), as evidenced in the comparison of the four panels of Fig. 5. This tells us that this dSph has an intrinsic residual velocity gradient, be it due to rotation (Battaglia et al. 2008) or tidal streaming, or that both proper motion measurements are incorrect. On the other hand, for Fnx and Car there is good agreement between the proper motions from stellar redshifts and those directly measured (see Walker et al. 2008), so that the gradients detected over the region sampled by the data are most likely due just to perspective effects. We look forward to the accurate proper motions that the Gaia satellite will deliver, because they will determine the amplitude and directions of "spurious" velocity gradients, and herewith also help unravel intrinsic gradients due to rotation or tidal effects.

Even if the origin of the velocity gradients is intrinsic rotation, the relatively low $v / \sigma$ values suggest that the internal kinematics of dSphs is dominated by random motions; we note that, in the hypothesis of internal rotation, this is the "projected" value, which should be corrected for the -unknowninclination of the angular momentum vector. 


\subsubsection{The second moment: l.o.s. velocity dispersion profiles}

Much care is invested in deriving robustly the l.o.s. velocity dispersion profiles $\sigma_{\text {l.o.s }}(R)$ of dSphs, not only because of the sensitivity of mass estimates to the value of the dispersion, but also because of the importance of the velocity dispersion profile shape in determining the DM mass distribution (see also Sec. 3) and providing possible hints on the dynamical status of the dSph.

There are two main schools of thought for deriving $\sigma_{\text {l.o.s. }}(R)$, which assume that: $i$ ) the dSph is in dynamical equilibrium; ii) the kinematic samples contain unbound dSph stars lost as a consequence of tides induced by the MW. These need to be removed as they are no longer tracers of the dSph galaxy potential. We will discuss the methodologies used in the literature for deriving $\sigma_{\text {l.o.s. }}(R)$ within either of the assumptions.

Dynamical equilibrium. The way the l.o.s. velocity dispersion profile is derived is very much related to the way of dealing with the foreground contamination. Hargreaves et al. (1994a) introduced a simple maximum likelihood procedure to evaluate simultaneously the systemic velocity $\bar{v}_{\mathrm{dSph}}$ and l.o.s. velocity dispersion $\sigma_{\mathrm{dSph}}$, assuming that the measurement errors and the velocities are Gaussianly distributed:

$$
L\left(v_{1}, v_{2}, \ldots, v_{N}\right)=\prod_{i} P\left(v_{i}\right)=\prod_{i} \frac{1}{\sqrt{2 \pi\left(\sigma_{i}^{2}+\sigma_{\mathrm{dSph}}^{2}\right)}} \exp \left[\frac{-\left(v_{i}-\bar{v}_{\mathrm{dSph}}\right)^{2}}{2\left(\sigma_{i}^{2}+\sigma_{\mathrm{dSph}}^{2}\right)}\right]
$$

where $L\left(v_{1}, v_{2}, \ldots, v_{N}\right)$ is the likelihood of observing a set of $N$ velocities

$v_{1}, v_{2}, \ldots, v_{N}, i$ goes from 1 to $N$, and $\sigma_{i}$ are the errors on the individual velocity measurements. The error ellipses on $\bar{v}_{\mathrm{dSph}}$ and $\sigma_{\mathrm{dSph}}^{2}$ are then given by steps down the peak of $\ln (L)$, i.e. $\ln (L)-1 / 2$ corresponds to the $68 \%$ confidence limit. They also provide the analytic formulas to use in an iterative scheme:

$$
\hat{\bar{v}}_{\mathrm{dSph}}=\frac{\sum_{i} w_{i} v_{i}}{\sum_{i} w_{i}}
$$

and

$$
\hat{\sigma}_{\mathrm{dSph}}^{2}=\frac{1}{N-1} \frac{\sum_{i}\left[\left(v_{i}-\bar{v}_{\mathrm{dSph}}\right)^{2}-\sigma_{i}^{2}\right] w_{i}^{2}}{\sum_{i} w_{i}^{2}},
$$

where the weights $w_{i}=1 /\left(\sigma_{i}^{2}+\sigma_{\mathrm{dSph}}^{2}\right)$ are updated in each iteration and the factor $1 /(N-1)$ is needed to make the estimated dispersion unbiased 
since the systemic velocity is evaluated at the same time. The $\sigma_{\text {l.o.s. }}(R)$ is then derived for each distance bin, where in most studies $\bar{v}_{\mathrm{dSph}}$ is assumed to be constant with $R$. While in absence of streaming motions this is a reasonable assumption, care should be taken when dealing with samples with asymmetric spatial coverage in presence of velocity gradients as this can result in $\bar{v}_{\text {dSph }}$ varying with $R$.

This simple iterative approach suffices when the number of MW contaminants expected within the velocity range used for membership is small in comparison to the number of genuine members, for example in the central regions (note that this is not necessarily the case for UFD). For samples extending also to the outer parts, it is more appropriate to explicitly take into account the contribution of MW stars when deriving the internal kinematic properties of the dSph.

For example Battaglia et al. (2008a) propose to derive $\bar{v}_{\mathrm{dSph}}$ and $\sigma_{\mathrm{dSph}}$ as a function of projected radius $R$ by maximizing the likelihood $L\left(v_{1}, \ldots, v_{N}\right)=$ $\prod_{i} P\left(v_{i}\right)$, with the individual probabilities given by:

$$
\begin{aligned}
P\left(v_{i} \mid \bar{v}_{\mathrm{dSph}}, \sigma_{\mathrm{dSph}}\right) & =\frac{N_{\mathrm{MW}}}{N_{\mathrm{TOT}}} f_{M W}\left(v_{i}\right)+ \\
& +\frac{N_{\mathrm{dSph}}}{N_{\mathrm{TOT}} \sqrt{2 \pi\left(\sigma_{\mathrm{dSph}}^{2}+\sigma_{i}^{2}\right)}} e^{-\frac{\left(v_{i}-\bar{v}_{\mathrm{dSph}}\right)^{2}}{2\left(\sigma_{\mathrm{dSph}}^{2}+\sigma_{i}^{2}\right)}} .
\end{aligned}
$$

The dSph LOSVD is assumed to be a Gaussian, while the MW LOSVD, $f_{\mathrm{MW}}(v)$ the sum of two Gaussians. This provides a good fit to the LOSVD of MW stars predicted by the Besançon model (Robin et al., 2003), in the direction to Scl and in the magnitude and color range of Scl target RGB stars. The surface number count profile of the target RGB stars in the dSph and the number density of MW contaminants in the same CMD region of the targets is derived from the wide-area ESO/WFI DART photometric data. This allows to determine the fraction of MW and Scl RGB stars per distance bin in elliptical annuli $\left(\frac{N_{\mathrm{MW}}}{N_{\mathrm{TOT}}}\right.$ and $\frac{N_{\mathrm{dSph}}}{N_{\mathrm{TOT}}}$, respectively). This method was also applied to the chemo-dynamical components of Scl (for all the details see Battaglia 2007).

Walker et al. (2009c) present an expectation maximization (EM) algorithm with applications to dSphs. The equations that identify this method are the probability that $M=1$ (i.e. that the star is a member), subject to 
the data $X_{i=1}^{N}$ and the prior constraints $p(a)$ (a priori membership fraction):

$$
\begin{aligned}
P_{M_{i}} & \equiv P\left(M_{I}=1 \mid X_{i}, a_{i}\right) \\
& =\frac{p_{\text {mem }}\left(X_{i}\right) p\left(a_{i}\right)}{p_{\text {mem }}\left(X_{i}\right) p\left(a_{i}\right)+p_{\text {non }}\left(X_{i}\right)\left[1-p\left(a_{i}\right)\right]}
\end{aligned}
$$

and the expected (log) likelihood given the data-set $S \equiv X_{i=1}^{N}$ :

$$
\begin{aligned}
E\left(\ln L\left(\xi_{\text {mem }}, \xi_{\text {non }} \mid S\right)\right. & =\sum_{i=1}^{N} P_{M_{i}} \ln \left[p_{\text {mem }}\left(X_{i}\right) p\left(a_{i}\right)\right] \\
& +\sum_{i=1}^{N}\left(1-P_{M_{i}}\right) \ln \left[p_{\text {non }}\left(X_{i}\right)\left[1-p\left(a_{i}\right)\right]\right]
\end{aligned}
$$

where $\xi_{\text {mem }}, \xi_{\text {non }}$ are the parameter set to evaluate, $p_{\text {mem }}$ and $p_{\text {non }}$ the probability distribution of members and non-members in the variable $X$. Before maximizing the expected $\ln (L)$, the $P_{M_{i}}$ are evaluated for all $i$.

In their formulation of the EM analysis applied to dSphs, the data-set consists of $S \equiv\left\{V_{i}, W_{i}, a_{i}\right\}_{i=1}^{N}$, where beside the individual l.o.s. velocities $V_{i}$, the variables are the Mg-index values of the stars $W_{i}$ and $a_{i}$ the elliptical distances. Except for the velocity probability distribution of nonmembers, which is estimated from the Besançon model, for members and the probability distributions in $\mathrm{W}$ are assumed to be Gaussian; finally, the only assumption made on $p(a)$ is that it decreases with radius. While in the approach of Battaglia et al. (2008a) the fraction of members as a function of projected distance is fully determined from the photometric data, without free parameters, here the $p\left(a_{i}\right)$ need to be evaluated for each $i$. The approach from Walker et al. has the advantage of being more flexible in the inclusion of new variables and mathematically more rigorous.

The membership probabilities obtained from the above likelihood approaches can be used to retain in the sample only stars with high probability values or as weights for subsequent calculations; for example, for the velocity dispersion profiles in Walker et al. (2009b) only stars with probability of membership larger than 0.95 are retained and then the $\sigma_{\text {l.o.s }}(R)$ is found using a maximum likelihood procedure analogous to that of Hargreaves et al. (1994a). In Bayesian extensions of the method, for example by Martinez et al. (2011) and Walker and Peñarrubia (2011), the probability of membership does not need to be directly evaluated. 
While previous works obtained flattish velocity dispersion profiles with cold points at the last measured radius for Sext, Draco and Ursa Minor (Kleyna et al., 2004; Wilkinson et al., 2004), now the general consensus is that dSphs have flat l.o.s. velocity dispersion profiles, with only gentle hints of a decline or a rise, as shown in Fig. 7. The approximately flat l.o.s. velocity dispersion profiles of dSphs are considered as the best evidence that the DM has a different spatial distribution than the stars, so that mass-follows-light models can be excluded, in the hypothesis of dynamical equilibrium.

Presence of unbound stars. The concern is that these might affect the inferred DM mass distributions and dynamical mass-to-light ratios. For example, Nbody simulations show that inclusion of unbound stars typically causes rising $\sigma_{\text {l.o.s }}(R)$ (or less often flat, e.g. Read et al., 2006; Klimentowski et al., 2007; Muñoz et al., 2008), even though the $\sigma_{\text {l.o.s }}(R)$ derived considering only bound stars is declining.

In the literature, the methodology used to get rid of unbound dSph stars is based on the virial theorem and was originally proposed to eliminate interlopers from clusters of galaxies (e.g. den Hartog and Katgert, 1996) and tested using dark-matter only N-body simulations of galaxy clusters in which random sub-sets of particles where chosen to represent galaxies (e.g. Wojtak et al. 2007); in other words in the situation where the number density of the tracers is proportional to that of the overall mass density (i.e. a "massfollows-light" model). Subsequently Klimentowski et al. (2007) tested the performance of the method using a high-resolution N-body simulation of a dSph formed within the tidal stirring framework, for which strong tidal stripping of the stellar component and a final (approximately) mass-followslight configuration were found. Using mock samples of l.o.s. velocities, they showed that $70-80 \%$ of the unbound stars from the tidal tails could be removed by the den Hartog and Katgert (1996) method, while only a very small fraction $(\sim 1 \%)$ of bound stars was mistaken for interlopers. When including foreground contamination from the MW, the fraction of correctly identified interlopers (both from the MW and from the tidal tails) decreases only of a few percent.

The above iterative procedure rejects objects whose velocity exceeds the maximum velocity available to a tracer particle (here a star) at a certain projected radius $R$. This maximum velocity is determined under the assumption that a star is either on a circular orbit with velocity $v_{\text {circ }}=\sqrt{G M(r) / r}$ or in free fall under the pull of the galaxy's potential with velocity $v_{\text {inf }}=\sqrt{2} v_{\text {circ }}$, 
given the mass profile $M(r)$ determined from the virial mass estimator:

$$
M\left(r_{j}\right) \equiv M_{\mathrm{VT}}\left(R_{j}\right)=\frac{3 \pi N}{2 G} \frac{\sum_{i}\left(v_{i}-\bar{v}\right)^{2}}{\sum_{i<j} 1 / R_{i j}}
$$

where - adapting to our case the definitions from den Hartog and Katgert (1996) $-M\left(r_{j}\right)$ is the mass enclosed within the distance to the star $r_{j}, R_{j}$ is the projection of $r_{j}, N$ is the number of stars in the sample, $R_{i j}$ are the projected distances of pairs of stars within a cylinder of radius $R$ around the center, $v_{i}$ and $\bar{v}$ have been defined above. Here $v_{\text {inf }}$ can be seen as the escape velocity from the mass interior to $r$ (Wojtak et al., 2007).

By applying this method for rejecting (putative) unbound stars, Łokas et al. (2008) and Lokas (2009) rederive the l.o.s. velocity dispersion profiles for Leo I, Car, Fnx, Scl and Sext using data from Mateo et al. (2008) and Walker et al. (2009a); they find gentle declines for all these galaxies and are able to explain the data with "mass-follows-light models" close to isotropic or mildly tangential. Note that the best fits still require considerable amounts of DM (see Sect. 3).

At present it is unclear how such method would perform for a system in which the mass-to-light ratio strongly increases with radius, for example if dSphs were embedded in an NFW or cored DM halo in dynamical equilibrium. To explore this, here we apply such a rejection procedure on the mock Scl model from Breddels et al. (2012) in which the stars are distributed as a Plummer sphere with $b=0.3 \mathrm{kpc}$, stellar mass $M_{*}=10^{6} \mathrm{M}_{\odot}$, velocity anisotropy $\beta=-0.5$, embedded within a NFW halo with scale radius $0.5 \mathrm{kpc}$ and $10^{8} \mathrm{M}_{\odot}$ mass enclosed within $1 \mathrm{kpc}$ radius. To the 50000 Scl mock stars, we add a mock MW foreground contamination, amounting to 5\%, 10\%, $20 \%$ and $50 \%$ of these stars, uniformly distributed in projected radius and \pm 40 $\mathrm{km} \mathrm{s}^{-1}$ around the systemic velocity. From the overall sample, we randomly extract 500 objects (genuine members and contaminants) and determine the velocity envelope using the infall velocity at $R, v_{\text {inf }}=\sqrt{2} v_{\text {circ }}$ evaluated at $r=R$. As shown in Fig. 6, after 3 iterations of the algorithm, several genuine members are identified as contaminants and the stars retained in the sample would lead to a declining $\sigma_{\text {l.o.s. }}(R)$. It still remains to be extensively tested how the rejection method based on the virial theorem would perform for a system in equilibrium for different functional forms of the DM halo and light distributions; here we have shown that a particular example of a dSph in equilibrium within extended and massive DM halos for which this rejection method would remove an important fraction of genuine members. 


\subsubsection{The higher moments: third and fourth}

The third moment has barely been used in the study of MW dSphs. The most detailed analysis has been carried out by Amorisco and Evans (2012c) by analyzing the angular behavior of asymmetric deviations from Gaussianity and found what appears to be an intrinsic rotation signal of $1 \mathrm{~km} \mathrm{~s}^{-1}$ about the minor axis in the Fnx dSph. No conclusions could be drawn for Scl, Car and Sext given the large error-bars and more limited sample sizes.

Therefore, in this section we focus mostly on the fourth moment because of the information it carries about the velocity anisotropy of the stars in dSphs. Knowledge of the velocity anisotropy allows to test possible formation scenarios of dSphs and helps break modeling degeneracies. For example, Merrifield and Kent (1990) show explicitly how two systems with the same surface density profile and l.o.s. velocity dispersion can have very different distribution functions and be embedded in different gravitational potentials. The only way to tell them apart is through a measure of the 4th moment of the LOSVD.

The 4th moment of the LOSVD has been quantified in various ways in the literature. Merrifield and Kent (1990) use the kurtosis, defined as

$$
\kappa_{\text {l.o.s }}(R)=\bar{v}_{\text {l.o.s }}^{4}{\overline{v^{2}}}_{\text {l.o.s. }}^{2} .
$$

A Gaussian function has a $\kappa_{\text {l.o.s }}=3$, while boxier LOSVD distributions have kurtosis $<3$ and more peaked LOSVD with longer tails have kurtosis $>3$. Merrifield and Kent (1990) state that to measure the kurtosis with an error $<10 \%$ a sample of 750 stars is needed, while if only 150 stars are available, then the error is $\sim 20 \%$, both with $90 \%$ confidence, which ought to allow the detection of gross variations in the velocity anisotropy.

Łokas and Mamon (2003a) define $K^{\prime}$, an unbiased estimator of the kurtosis for a sample of $n$ l.o.s. velocity measurements $v_{i}$

$$
K^{\prime}=\frac{3}{C} K
$$

with $K$ being

$$
K=\frac{\frac{1}{n} \sum_{i=1}^{n}\left(v_{i}-\bar{v}\right)^{4}}{\left(S^{2}\right)^{2}}
$$

and

$$
\bar{v}=\frac{1}{n} \sum_{i=1}^{n} v_{i}, S^{2}=\frac{1}{n} \sum_{i=1}^{n}\left(v_{i}-\bar{v}\right)^{2} .
$$


$K^{\prime}$ is introduced because $K$ was found to underestimate the kurtosis in Monte Carlo realizations of a Gaussian distribution. Since the sampling distribution of $K$ is strongly skewed, Lokas and Mamon (2003a) also suggest to use the function $k=\left[\log K^{\prime}\right]^{1 / 10}$, which follows a Gaussian sampling distribution, valid also for weakly non-Gaussians LOSVD (Eokas et al., 2005). The value of $C$ and the standard error on $k$ vary with the number of objects (per bin) and become $C=2.75,2.89,2.93$ for $N=40,100,200$, while the corresponding sampling error for $k$ is 0.02, 0.0124, 0.009 (see also Łokas, 2009). For sample sizes with a few hundreds objects per bin, the value of the kurtosis as given by Eq. (14) remains underestimated by a few percent. $k=0.93$ corresponds to a Gaussian distribution, while flatter (more peaked) distributions have $k<0.93$ (> 0.93).

More recently, Breddels et al. (2012) defined a 4-th moment estimator as:

$$
\hat{\mu}_{4}=\sum_{i=1}^{n}\left(v_{i}+\epsilon_{i}\right)^{4}-3 s_{2}^{2}+6 \mu_{2} s_{2}
$$

where $\epsilon_{i}$ is the velocity error for star $i$ (this term accounts for the noise of the measured velocity $\left.v_{i}\right), s_{2}$ is the average of the measured squared velocity errors, and the second moment of the LOSVD $\mu_{2}$ is approximated by the estimator $\hat{\mu_{2}}$ :

$$
\hat{\mu_{2}}=\frac{1}{n} \sum_{i=1}^{n}\left(v_{i}+\epsilon_{i}\right)^{2}-s_{2} .
$$

This estimator has the same distribution of values as the kurtosis.

One problem of the kurtosis, in its various definitions, is that it heavily weighs the tails of the LOSVD (since it is proportional to $v^{4}$ ), and so for example, contamination by foreground/unbound stars can be an issue. In this sense it has been argued that the use of Gauss-Hermite moments is generally preferable as these are less dependent on the wings of the distribution, and because they can be derived more accurately.

Mainly in the context of studying the properties of elliptical galaxies, Gerhard (1993) and van der Marel and Franx (1993) showed that the LOSVD $(L(v))$ from absorption line spectroscopy could be well described with just a few terms of the expansion in Gauss-Hermite functions, typically just up to order 4. Since Gaussians provide good first-order approximations to the $L(v)$, van der Marel and Franx (1993) introduced the following definition for 
the Gauss-Hermite serie:

$$
L(v)=[\gamma \alpha(w) / \sigma]\left\{1+\sum_{j=3}^{N} h_{j} H_{j}(w)\right\}, \text { with } w=(v-V) / \sigma .
$$

where $\gamma, \mathrm{V}, \sigma$ are the line strength, mean radial velocity and velocity dispersion, respectively, of the best-fitting Gaussian to the observed $L(v)$; the functions $H_{j}(w)$ are Hermite polynomials (for the definition see van der Marel and Franx, 1993); $h_{0}, h_{1}, h_{2}$ are set to $(1,0,0)$ and $h_{3}, \ldots, h_{N}$ contain the shape information; specifically, $h_{3}$ and $h_{4}$ measure asymmetric and symmetric deviations from Gaussianity. Here $h_{4}>0$ corresponds to a peaked distribution, while $h_{4}<0$ to a more flat-topped distribution. They also show that negative $h_{4}$ corresponds typically to tangential anisotropy, for a similar set of gravitational potentials and distribution functions as explored by Gerhard (1993).

Amorisco and Evans (2012c) have tested how the discrete nature of dSph data-sets affects the reliable determination of the Gauss-Hermite moments of the LOSVD. They estimate that 200 stars are necessary not to be dominated by noise due to limited sampling: in this case, the shot noise on $h_{3}$ and $h_{4}$ is $\sim 0.05$. If one wishes to study how the moments vary as a function of distance, of course the error refers to the number of stars per bin.

These authors also argue that the Gauss-Hermite formalism cannot be readily applied to the kinematic data available for the dSphs because of the difficulties in accounting for the heterogeneous observational uncertainties and probability of membership associated to each star, and for the fact that the LOSVD is a convolution of the intrinsic $L(v)$ with the measurement errors. They also note that this expansion does not define a proper probability density function since the Gauss-Hermite series is not always positive definite everywhere. Therefore they introduce a set of quantities to characterize asymmetric and symmetric deviations from a Gaussian distribution, and which they argue are less sensitive to shot noise than the Gauss-Hermite moments (roughly a factor 2 smaller for $h_{4}$ for a sample of 800 stars).

The fourth moment of the LOSVD of MW dSphs has been derived and analyzed for most classical dSphs, although not always used in the mass modeling. At present the results for Leo I are contrasting: Sohn et al. (2007) detect a broad and skewed LOSVD, in particular for stars at large angular separations on the West side of the galaxy; this has not been confirmed by Mateo et al. (2008), who find that the skewness and kurtosis are consistent 
with a Gaussian distribution. It is unclear if the different results may be due to the systematic velocity differences between the data-sets used in the two works. Łokas et al. (2008) have re-analyzed the sample of Mateo et al. (2008) finding that the LOSVD departs from Gaussian and becomes rather irregular at $R>6$ arcmin, but consistent with a Gaussian at smaller $R$.

For Car, Fnx, Scl and Sext, Łokas (2009) apply the interloper removal scheme based on the virial estimator to the Walker et al. (2009a) sample and find either flat $k(R)$ very close to the value expected for a Gaussian distribution or slightly declining $k(R)$ with values in the range 0.90-0.95; the latter imply very mild tangential anisotropy, consistent within $1 \sigma$ with an isotropic velocity ellipsoid. Fourth moment values corresponding to mild tangential anisotropies are found by Breddels and Helmi (2013) for Scl and by Amorisco and Evans (2012c) for Fnx, assuming that the dSphs are in dynamical equilibrium.

On the other hand, Amorisco and Evans (2012c) find a preference for small positive values of $h_{4}$ (and of their new parameter measuring symmetric deviations from Gaussianity) for Scl, and also Sext. The contrasting results may be attributed to the different ways of dealing with membership, as both works used similar data-sets as Breddels et al. (2012). Also Łokas et al. (2005) found that the values (and profile shapes) of the unbiased kurtosis estimator vary according to the treatment of interlopers, going from flat around the value expected for a Gaussian distribution, to slightly declining or slightly rising.

From the above it is clear that it is important to model carefully the stars' membership, as this can impact the conclusions on the orbital structure of stars in dSphs. The situation could likely be improved by using the probability of membership only as a weight when deriving the kinematic properties, rather than to use it for eliminating stars from the sample.

Notwithstanding the above issues, the current measurements suggest that the LOSVD of stars in dSphs are not dramatically different from Gaussians in most cases, so that one can conclude that the velocity ellipsoid is neither strongly radial nor strongly tangential.

\subsubsection{Chemo-dynamical components}

Several classical dSphs exhibit spatial variations in their metallicity distribution functions, with the mean metallicity being higher in the inner regions and lower in the outer parts (e.g. Tolstoy et al. 2004, Battaglia et al. 2006, Koch et al. 2006, Faria et al. 2007, Battaglia et al. 2011). 
For Scl, Fnx and Sext, the internal kinematics are found to be linked to the metallicity, in that the "metal-rich" stars are more centrally concentrated and exhibit a lower $\sigma_{\text {l.o.s }}$ than the metal-poor stars which form a more extended population (Tolstoy et al. 2004, Battaglia et al. 2006, Battaglia et al. 2011). This is clearly seen in Fig. 8 for the Scl dSph, where the $\sigma_{\text {l.o.s }}$ of stars with $[\mathrm{Fe} / \mathrm{H}]>-1.5$ is also found to decline with $R$, while for those with $[\mathrm{Fe} / \mathrm{H}]<-1.7$ it stays approximately constant (see also Walker and Peñarrubia, 2011).

The analysis of the kinematic properties of these "chemo-dynamical" stellar components paints in some cases a very complex evolutionary picture, as for the Fnx dSph. The analysis of Amorisco and Evans (2012a) suggests that this system is best described by the superposition of 3 chemo-dynamical components, increasingly more metal-rich, more spatially concentrated and with colder kinematics. The authors detect what appears to be a misalignment of the angular momentum of intermediate-metallicity and metal-poor stars. Such a detailed analysis would not have become possible without the large spectroscopic data-sets with metallicity and kinematical information available nowadays. Furthermore, from a dynamical modeling perspective, the presence of different "chemo-dynamical" stellar components have provided interesting new constraints on the DM mass distribution of dSphs (see Sect. 3.5.).

\section{Dynamical modeling}

The techniques to model the internal dynamics of spheroidal systems have long been in place. However, their application to nearby dwarf spheroidals has only really taken off in the last decade, with the need for more sophisticated approaches thanks to the manifold increase in data samples. In this section we review the methods used, briefly discuss their limitations and the results obtained thus far for these systems. Table 1 gives an overview of the various modeling techniques applied to the MW dSphs.

We divide this Section according to the groups of methods that have been used so far. In general, we can broadly classify methods on whether they are parametric, i.e. they assume a family of models, or non-parametric, in which the distribution function is expressed in more general terms, for example as an expansion of basis functions. Most works attempt to fit the moments of the velocity distributions, while the use of the velocities and positions of 
individual stars to determine the likelihood of a given model (also known as discrete modeling) has been explored to a lesser extent in the literature.

\subsection{Modeling with the Jeans Equations}

To a very good approximation, a dwarf galaxy may be considered a collisionless system. The internal structure of such a dynamical system can be described through its distribution function $f(\mathbf{x}, \mathbf{v}, t)$, which in the collisionless case, obeys the Boltzmann equation

$$
\frac{\partial f}{\partial t}+\mathbf{v} \cdot \nabla_{x} f-\nabla_{x} \Phi \cdot \nabla_{v} f=0
$$

where $\Phi(\mathbf{x})$ is the total gravitational potential of the system (including stars and dark matter contributions Binney and Tremaine, 2008). For our purposes $f(\mathbf{x}, \mathbf{v}, t)$ describes the probability of finding a star with a given position $\mathbf{x}$, and velocity $\mathbf{v}$ at time $t$.

In general we assume that the distribution function is time-independent (see Sec. 1.3), so that the first term in this equation may be dropped. Deriving the distribution function from Eq. (19) by comparison to observations is not straightforward (see below), so a commonly used approach is to take moments of this equation, and compare these moments to observables, since also low-order moments are easily measured from observations.

The zero-th moment corresponds to the continuity equation in hydrodynamics, and it is generally not used in data-model comparison. The first moment is obtained by multiplying Eq.(19) by $v_{j}$ and integrating over all velocities. The resulting equation is

$$
\frac{\partial \nu\left\langle v_{i} v_{j}\right\rangle}{\partial x_{i}}+\nu \frac{\partial \Phi}{\partial x_{j}}=0
$$

where $\nu(\mathbf{x})$ is the stellar density, i.e. $\nu(\mathbf{x})=\int d^{3} v f$, and the brackets \langle\rangle denote moments, e.g. here $\left\langle v_{i} v_{j}\right\rangle=\int d^{3} v v_{i} v_{j} f$. Eq. 20 represents a set of 3 equations known as the Jeans equations. These are useful because they relate to observables, however, it should be born in mind that this is not a closed set of equations, in the sense that even if we knew the potential and the density, to derive the streaming (mean) velocities (3 components) and the full velocity ellipsoid (6 independent quantities), we only have 4 equations, i.e. the continuity and the Jeans equations. Although it is possible to use higher moments of the Boltzmann equation, this tends to be more cumbersome. 
Higher moments are also difficult to measure observationally reliably, and nonetheless the use of closure relations would still be necessary. Therefore, typically, as we shall see below, certain assumptions are made, regarding for example the form of the velocity ellipsoid, to find a solution to the system.

The distribution function of a steady state system depends on the integrals of motion. If the potential is time-independent, then the energy $E$ is an integral of motion. For a spherical system, all components of the angular momentum $\mathbf{L}$ are conserved, while if the system is axisymmetric, then only $L_{z}$ will be, but a third integral $I_{3}$ might exist. Therefore, in non-rotating spherical systems, the distribution function can be a function $f(E)$ or $f(E, L)$. Although it is possible for a spherical system to rotate (Lynden-Bell, 1960), in which case the distribution function will be of the form $f(E, \mathbf{L})$ this is not the most general configuration. Rotation would be more natural in the axisymmetric case, when $f\left(E, L_{z}\right)$, i.e. there is a preferred axis (that about which the system rotates). As discussed in Sec. 2.3.2, there is evidence of small velocity gradients in the dSphs, however, their origin is unclear, and in many cases these can be explained by projection effects. Therefore, in the rest of this review we assume that our systems to do not rotate. In that case, the second moment and the variance of the velocity distribution are equal (after subtraction of the gradient), and we refer to these interchangeably.

\subsubsection{Jeans equations for spherical systems}

In the case of a spherical system, only one of the Jeans equations is nontrivially zero, and it relates the 2 nd moment of the radial velocity $\left\langle v_{r}^{2}\right\rangle$, the stellar density $\nu(r)$, the velocity anisotropy $\beta(r)=1-\left(\left\langle v_{\theta}^{2}\right\rangle+\left\langle v_{\phi}^{2}\right\rangle\right) /\left(2\left\langle v_{r}^{2}\right\rangle\right)$, and the total gravitational potential $\Phi(r)$ as follows:

$$
\frac{\mathrm{d}\left(\nu\left\langle v_{r}^{2}\right\rangle\right)}{\mathrm{d} r}+2 \frac{\beta}{r} \nu\left\langle v_{r}^{2}\right\rangle=-\nu \frac{\mathrm{d} \Phi}{\mathrm{d} r} .
$$

An equivalent, often useful form of this equation is

$$
\frac{G M(r)}{r}=\left\langle v_{r}^{2}\right\rangle\left(\gamma_{*}-2 \beta-\alpha\right)
$$

where $r$ is the spherical radius, $\gamma_{*}=-d \log \nu / d \log r$ and $\alpha=d \log \left\langle v_{r}^{2}\right\rangle / d \log r$. For example, if the radial velocity and stellar density have been measured, and we make an assumption on the velocity anisotropy $\beta$, we may be able to derive the mass distribution (gravitational potential) of the system. This is the most frequently used approach. The velocity ellipsoid can be isotropic, in 
which case $\beta=0$, tangentially or radially anisotropic, when $\beta<0$ or $\beta>0$ respectively, and will in the most general case, vary with radius. In the case of $\beta=0$, this implies that the velocity distribution is ergodic, i.e. it is only a function of energy $f=f(E)$, while for anisotropic systems, $f=f(E, L)$.

The above equations highlight a degeneracy between mass and anisotropy (if the stellar density is perfectly known from observations; otherwise this also enters the degeneracy). This is most easily seen if we assume that $\beta$ is constant with radius. In that case, Equation (21) reduces to (Binney and Tremaine, 2008)

$$
\left\langle v_{r}^{2}(r)\right\rangle=\frac{1}{r^{2 \beta} \nu(r)} \int_{r}^{\infty} \mathrm{d} r^{\prime} r^{2 \beta} \nu\left(r^{\prime}\right) \frac{\mathrm{d} \Phi}{\mathrm{d} r^{\prime}} .
$$

We thus see directly that different combinations of the mass distribution, density and anisotropy might conspire to produce the same velocity dispersion profile in the radial direction. The situation is worsened by the fact that generally one deals with projected quantities, as discussed below.

A way to reduce the degeneracy is to use higher moments, in particular, the 4th moment equations are obtained by multiplying Eq. $(19)$ by $v_{r}^{3}$ and $v_{r} v_{t}^{2}$ and integrating over velocity space (see Merrifield and Kent, 1990):

$$
\frac{\mathrm{d}\left(\nu\left\langle v_{r}^{4}\right\rangle\right)}{\mathrm{d} r}-3 \frac{\nu}{r}\left\langle v_{r}^{2} v_{t}^{2}\right\rangle+\frac{2}{r} \nu\left\langle v_{r}^{4}\right\rangle+3 \nu\left\langle v_{r}^{2}\right\rangle \frac{\mathrm{d} \Phi}{\mathrm{d} r}=0
$$

and

$$
\frac{\mathrm{d}\left(\nu\left\langle v_{r}^{2} v_{t}^{2}\right\rangle\right)}{\mathrm{d} r}-\frac{\nu}{r}\left\langle v_{t}^{4}\right\rangle+\frac{4}{r} \nu\left\langle v_{r}^{2} v_{t}^{2}\right\rangle+\nu\left\langle v_{t}^{2}\right\rangle \frac{\mathrm{d} \Phi}{\mathrm{d} r}=0 .
$$

If one assumes that the distribution function is of the form $f(E, L)=$ $f_{0}(E) L^{-2 \beta}$, it can be shown that the anisotropy is constant, and these equations simplify significantly to (Eokas, 2002)

$$
\frac{\mathrm{d}\left(\nu\left\langle v_{r}^{4}\right\rangle\right)}{\mathrm{d} r}+\frac{2 \beta}{r} \nu\left\langle v_{r}^{4}\right\rangle+3 \nu\left\langle v_{r}^{2}\right\rangle \frac{\mathrm{d} \Phi}{\mathrm{d} r}=0
$$

whose solution may be expressed as

$$
\left\langle v_{r}^{4}(r)\right\rangle=\frac{3}{r^{2 \beta} \nu(r)} \int_{r}^{\infty} \mathrm{d} r^{\prime} r^{2 \beta} \nu\left(r^{\prime}\right)\left\langle v_{r}^{2}\left(r^{\prime}\right)\right\rangle \frac{\mathrm{d} \Phi}{\mathrm{d} r^{\prime}} .
$$

As discussed above, the intrinsic moments are not directly accessible to the observer, and only projected moments of the line-of-sight velocity distribution and stellar density profile are measurable. Following Merrifield and 
Kent (1990) these projected moments take the form

$$
\begin{gathered}
\mu(R)=2 \int_{R}^{\infty} \nu(r) \frac{r \mathrm{~d} r}{\left(r^{2}-R^{2}\right)^{1 / 2}}, \\
\left\langle v_{\mathrm{los}}^{2}(R)\right\rangle=\frac{2}{\mu} \int_{R}^{\infty} \nu(r)\left[\left(1-\frac{R^{2}}{r^{2}}\right)\left\langle v_{r}^{2}\right\rangle+\frac{1}{2} \frac{R^{2}}{r^{2}}\left\langle v_{t}^{2}\right\rangle\right] \frac{r \mathrm{~d} r}{\left(r^{2}-R^{2}\right)^{1 / 2}}, \\
\left\langle v_{\mathrm{los}}^{4}(R)\right\rangle=\frac{2}{\mu} \int_{R}^{\infty} \nu(r)\left[\left(1-\frac{R^{2}}{r^{2}}\right)^{2}\left\langle v_{r}^{4}\right\rangle+3 \frac{R^{2}}{r^{2}}\left(r^{2}-R^{2}\right)\left\langle v_{r}^{2} v_{t}^{2}\right\rangle+\frac{3}{8} \frac{R^{4}}{r^{4}}\left\langle v_{t}^{4}\right\rangle\right] \\
\times \frac{r \mathrm{~d} r}{\left(r^{2}-R^{2}\right)^{1 / 2}} .(30)
\end{gathered}
$$

Here $R$ denotes the projected radial distance. Expressed in terms of the anisotropy $\beta$ these equations take the form

$$
\begin{gathered}
\left\langle v_{\text {los }}^{2}(R)\right\rangle=\frac{2}{\mu} \int_{R}^{\infty} \nu(r)\left(1-\beta \frac{R^{2}}{r^{2}}\right)\left\langle v_{r}^{2}\right\rangle \frac{r \mathrm{~d} r}{\left(r^{2}-R^{2}\right)^{1 / 2}}, \\
\left\langle v_{\text {los }}^{4}(R)\right\rangle=\frac{2}{\mu} \int_{R}^{\infty} \nu(r)\left\langle v_{r}^{4}\right\rangle g(r, R, \beta) \frac{r \mathrm{~d} r}{\left(r^{2}-R^{2}\right)^{1 / 2}},
\end{gathered}
$$

where

$$
g(r, R, \beta)=1-2 \beta \frac{R^{2}}{r^{2}}+\beta(1+\beta) / 2 \frac{R^{4}}{r^{4}},
$$

(Eokas and Mamon, 2003b). Eq. (32) is valid for the specific form of the distribution function that leads to a constant anisotropy, while Eq. (31) is more general.

In the recent past, Jeans modeling has been the most frequently used method to estimate the mass content of dSphs (Łokas, 2001; Kleyna et al., 2001; Koch et al., 2007b; Gilmore et al., 2007; Walker et al., 2007b; Battaglia et al. 2008a). For simplicity, many of the works assumed a constant anisotropy, and typically only the second moment is fit using the Jeans equation (although see below). The first modeling attempts already showed that mass following light models could not fit the relatively flat velocity dispersion profiles observed, and that extended dark matter halos were needed, for example in the case of Draco (Kleyna et al., 2001).

More recently, the focus has shifted to the type of dark matter halos that could host dSphs. For example, Gilmore et al. (2007) assumed the velocity 
ellipsoid to be isotropic $(\beta=0)$, a cored light surface density distribution and a flat (inner) l.o.s. velocity dispersion profile, and found that dSphs could be embedded in cored or cuspy dark matter halos (but shallower than the singular isothermal sphere). Walker et al. (2007b) assume NFW profiles and constant anisotropy together with an exponentially declining surface brightness distribution. These authors fit the total mass $M_{\mathrm{vir}}$ and (constant) anisotropy $\beta$, and assume a particular value for the concentration from the virial mass-concentration relationship found in cosmological N-body simulations (e.g. Bullock et al., 2001; Macciò et al., 2007). It is important to stress that a quantity such as the total mass is not well constrained, but what is better constrained is the mass within a given radius (within the region spanned by the dataset). Such a quantity is less sensitive to the functional form of the density profile, and therefore preferable. Although the virial mass may be considered just another (free) parameter of the fit, its meaning as representing the total mass of the system is actually an extrapolation.

Walker et al. (2009b, 2010) have extended the modeling of their sample of $\mathrm{dSphs} \mathrm{to} \mathrm{allow} \mathrm{for} \mathrm{more} \mathrm{general} \mathrm{forms} \mathrm{of} \mathrm{the} \mathrm{density} \mathrm{profile} \mathrm{of} \mathrm{the} \mathrm{dark} \mathrm{matter}$ $\left(\rho \propto 1 /\left(x^{\gamma}\left(1+x^{\kappa}\right)^{(3-\gamma) / \kappa}\right)\right.$, with $\gamma, \kappa \geq 0$, i.e. as in Eq. (3) with an outer slope $\alpha=3$ ), while still assuming constant anisotropy. They use a Monte Carlo Markov Chain (MCMC) method to explore the space of parameters and find the best fit models. The results are shown in Fig. 7. An interesting finding is that they can strongly constrain the mass at the projected halflight radius $r_{\text {half }}$ (the projected radius enclosing half of the total luminosity). Therefore, they also derive the circular velocity at $r_{\text {half }}, V_{\text {half }}$, and hence place lower limits on $V_{\max }$. This is only a lower limit because there is a degeneracy between $V_{\max }$ and the scale radius $r_{s}$ of NFW profiles, as there are many such profiles consistent with a given measurement of $V_{\text {half }}$ (Peñarrubia et al. 2008a) 7 .

Łokas (2009) performed Jeans modeling of Car, Fnx, Sext and Scl using the 2nd and 4th moments of the l.o.s. velocity distribution. As explained in Sec. 2.3.3 an important difference with work by other authors is the procedure to deal with interloper removal, which leads to velocity dispersion profiles that decrease with radius. As a consequence, Łokas (2009) finds

\footnotetext{
${ }^{7}$ However, this degeneracy can be broken by measuring the velocity dispersion profile over a large extent in radius, as shown by Breddels and Helmi (2013). There is a second, more difficult to break degeneracy between the slope/functional form of the density profile and $r_{s}$.
} 
systematically lower masses than other authors, and that models in which mass follows light can in fact, fit the derived observables. However, the $M / L$ derived are still much greater than expected from standard stellar populations (generally much greater than 10, see their Table 2). In the case of Draco, Eokas et al. (2005) found that the anisotropy was mildly tangential for a model with a $r^{-1}$ density profile (with an exponential cut-off). This is consistent with the Jeans model by Walker et al. (2009b) and also with Jardel and Gebhardt (2012) orbital based Schwarzschild model of the system (within $\sim 1 \sigma$, see below). Although the use of the 4th moment leads to a better constraint on the model parameters, its effect is relatively minor, and the solutions found are rather similar to those in which only the second moment is used. The reason for this may be attributed to the fact that the uncertainties on the measured kurtosis are large (samples are still small to measure moments very reliably), and that the differences with a Gaussianlike velocity distribution are not very big, in which case the 2 nd moment suffices to characterize the LOSVD, as discussed in Sec. 2.3.4.

\subsubsection{Jeans equations for axisymmetric systems}

The light distribution of dwarf spheroidals is not really spherically symmetric, nor are the shapes of dark matter halos predicted by $\Lambda$ CDM simulations, so Jeans modeling as described in the previous section is not necessarily justified. The next natural degree of complexity in the modeling of dSphs is to allow for axisymmetry. Although one might attempt to use fully triaxial models, this might not be necessary given that there is no clear evidence of twisting in the isophotes of dSphs (except for Car, which is likely to be affected by tides, see - Battaglia et al., 2012, and references therein). Furthemore, the analysis of the shapes of dark matter satellites in Milky Way-like simulations (Kuhlen et al., 2007; Vera-Ciro et al., 2013), indicate that these are not strongly triaxial (with axis ratios at $\sim 1 \mathrm{kpc}$ derived from the inertia tensor of $\langle c / a\rangle \sim 0.6$ and $\langle b / a\rangle \sim 0.75$, and $\langle c / a\rangle \sim 0.8$ and $\langle b / a\rangle \sim 0.9$ farther out).

In the axisymmetric case, we take the velocity moments of Eq. (19) in the directions $R, \phi$ and $z$. This results in the following equations respectively

$$
\begin{gathered}
\frac{\partial \nu\left\langle v_{R}^{2}\right\rangle}{\partial R}+\frac{\partial\left(\nu\left\langle v_{R} v_{z}\right\rangle\right)}{\partial z}+\nu\left(\frac{\left\langle v_{R}^{2}\right\rangle-\left\langle v_{\phi}^{2}\right\rangle}{R}+\frac{\partial \Phi}{\partial R}\right)=0, \\
\frac{1}{R} \frac{\partial\left(R \nu\left\langle v_{R} v_{z}\right\rangle\right)}{\partial R}+\frac{\partial \nu\left\langle v_{z}^{2}\right\rangle}{\partial z}+\nu \frac{\partial \Phi}{\partial z}=0,
\end{gathered}
$$


and

$$
\frac{1}{R^{2}} \frac{\partial\left(R^{2} \nu\left\langle v_{R} v_{\phi}\right\rangle\right)}{\partial R}+\frac{\partial \nu\left\langle v_{z} v_{\phi}\right\rangle}{\partial z}=0
$$

relating the intrinsic properties of the system. As in the spherical case, this set of equations is not closed, unless we make assumptions about the shape of the velocity ellipsoid, or the form of the distribution function. A typical consideration is to assume that the distribution function is of the form $f\left(E, L_{z}\right)$, in which case the mixed moments vanish, the last equation is trivially satisfied, and $\left\langle v_{z}^{2}\right\rangle=\left\langle v_{R}^{2}\right\rangle$. In that case the other two equations reduce to

$$
\frac{\partial \nu\left\langle v_{z}^{2}\right\rangle}{\partial z}+\nu \frac{\partial \Phi}{\partial z}=0
$$

and

$$
\frac{\partial \nu\left\langle v_{R}^{2}\right\rangle}{\partial R}+\nu\left(\frac{\left\langle v_{R}^{2}\right\rangle-\left\langle v_{\phi}^{2}\right\rangle}{R}+\frac{\partial \Phi}{\partial R}\right)=0 .
$$

More generally, the velocity dispersions in the radial $R$ and vertical $z$ directions will not be identical (Cappellari, 2008, and references therein), but we might express $\left\langle v_{R}^{2}\right\rangle=b\left\langle v_{z}^{2}\right\rangle$, where $b$ is a constant. Vera-Ciro et al. (2013) have shown the $\Lambda \mathrm{CDM}$ subhalos have constant $\beta_{z}=1-\left\langle v_{z}^{2}\right\rangle /\left\langle v_{R}^{2}\right\rangle$ along the minor axis, but that there exists a weak trend as function of distance along the major axis, with $\beta_{z} \sim+0.2$ to -0.2 from the center to the outskirts, implying that the simple assumption by Cappellari (2008) is not significantly violated.

Naturally, these are intrinsic quantities while we only have projected moments for the dSphs at our disposal. The equations relating these are given in Cappellari (2008) and Hayashi and Chiba (2012) for the cases discussed by these authors. Clearly one of the uncertainties in the projection is the inclination of the object with respect to the observer, and this is therefore generally an outcome of the modeling procedure. Another assumption often made is that the density of the tracer has the same orientation and symmetry as that of the dark matter halo. For dSph galaxies, this is justifiable since this is the dominant contributor to the gravitational potential and the stars are simply tracers.

Hayashi and Chiba (2012) show how the line of sight velocity profiles vary when objects are observed along the major and minor axis for various density profiles and flattenings in the light distribution. They find that the effect of a more flattened stellar system while keeping the dark matter halo shape fixed, is to produce wavy features in the l.o.s. velocity dispersion profile 
along the major axis, especially in the central regions (see their Fig. 2), and that the shapes are also different along the minor axis, naturally falling off faster with radius. The LOSVD amplitude and exact shape change depending on the dark matter density profile. These authors have also measured the velocity dispersion profiles for Car, Fnx, Sext, Draco, Leo I and Scl, and there is only a clear difference along the major and minor axis of the light distribution for Car and possibly Sext at large radii. From their modeling the authors conclude that the shapes of the dark halos are very flattened $(Q \sim 0.3-0.4)$ for most of their dSphs, much more than expected in $\Lambda$ CDM, and also significantly more than the light itself $(q \sim 0.65-0.8)$. This could be related to the assumption of semi-isotropy $\left(\left\langle v_{z}^{2}\right\rangle=\left\langle v_{R}^{2}\right\rangle\right)$. There is a strong degeneracy between $\beta_{z}$ and $Q$, since a flattening of the velocity ellipsoid $\beta_{z}>0$ has a very similar effect to a flattening of the halo $Q<1$. Moreover a very small change in $\beta_{z}$ anisotropy can mimic a major change in the halo shape, as shown in Figure 9. It would clearly be valuable to apply the more general modeling by Cappellari (2008) on the dSphs to establish the reliability/confidence of the conclusions.

Another interesting result from Hayashi and Chiba (2012) is that the total mass of the dSphs enclosed within a spheroid with major-axis length of $300 \mathrm{pc}$ varies from $10^{6}-10^{7} M_{\odot}$, i.e. these masses are lower than those estimated from spherical models by a factor that is roughly proportional to the flattening $Q$ (estimated by Hayashi and Chiba, 2012) .

\subsubsection{Other interesting results based on the Jeans equations}

As discussed in previous sections, modeling using the spherical Jeans equations requires assumptions on the functional form of the anisotropy and of the density profile of the dark matter halo of the system. The solution to e.g. Eq. (29) then gives us the parameters of the profile (e.g. a mass/density and scale radius, and an anisotropy). More generally, also the shape of the density profile may be allowed to vary, as in e.g. Strigari et al. (2007b); Walker et al. (2009b).

A common mass scale?. In two thorough studies, Strigari et al. (2007b, 2008) used MCMC numerical methods to explore a large range of models for the dark halos hosting dSphs. These authors found that of all the parameters describing the model, the mass within $300 \mathrm{pc}$ was a robust and well determined quantity, that was roughly independent of anisotropy or shape parameters. In an immediate application of this result, Strigari et al. (2008) found that 
most dSphs must be embedded in dark matter halos of similar mass within this radius $M_{300} \sim 1-2 \times 10^{7} M_{\odot}$, despite the fact that they span several orders of magnitude in luminosity. Although these results have been refined, especially for the ultrafaint dSphs, which do not even extend up to $300 \mathrm{pc}$ (and hence this $M_{300}$ is an extrapolation), in general the $M_{300}$ is confirmed to be a very weak function of luminosity $M_{300} \propto L^{0.03 \pm 0.03}$ (Rashkov et al. 2012).

Robust measurement of $M\left(r_{1 / 2}\right)$. The virial theorem as well as the Jeans equations actually offer a plausible explanation for why the mass at a particular radius may be estimated reliably from the LOSVD only. The virial theorem tells us that $M_{\text {tot }}=\sigma_{\text {tot }}^{2} r_{g} / G$ where $r_{g}$ is the gravitational radius of the system (see Eq. (2.42) of Binney and Tremaine, 2008). On the other hand, Wolf et al. (2010) have shown analytically using the Jeans equation, that at the radius at which the slope of the stellar density profile $d \log \nu / d \log r=-\gamma_{*}=-3$, the mass is very well constrained independently of the anisotropy of the system. Thus

$$
M\left(r_{-3}\right)=3 \frac{\left\langle\sigma_{\text {los }}^{2}\right\rangle r_{-3}}{G}
$$

for a system with a flat velocity dispersion profile. Since most of the dSphs have such relatively flat profiles, Wolf et al. (2010) and also Walker et al. (2009b, 2010) in their MCMC analysis of the Jeans equation have been able to confirm this analytic result. In general, however, instead of estimating the radius $r_{-3}$, Wolf et al. (2010) use the half-light radius $r_{1 / 2}$ since the two are very similar for most profiles used to model the light distribution in dSphs. Note that this is the $3 \mathrm{D}$ radius containing half of the total luminosity of the system, and not the effective radius obtained from the surface brightness profiles nor the $2 \mathrm{D}$ projected radius containing half of the luminosity, $r_{\text {half }}$ in Walker et al. (2010).

These relations are also useful for ultrafaint dSphs, provided these systems are in dynamical equilibrium. The sample sizes for most of these systems are too sparse to warrant a full dynamical model so general scalings as those just described may be more useful. See also An and Evans (2011) for more information on the theory of virial mass estimators.

General constraints on the $d f$. Not every solution to the Jeans equation has an associated distribution function that is physical, i.e. positive everywhere. 
This is why it is important to find additional conditions that can help identify when the assumptions made to solve the Jeans Equations will lead to plausible (physical) solutions.

An and Evans (2006, 2009) and Evans et al. (2009) use the Jeans equations to explore the asymptotic relations between the anisotropy $\beta$, the logarithmic slope of the light distribution $\gamma_{*}$ and that of the underlying dark matter density profile near the center of a spherical system $\gamma_{D M}$. They show that, if the tracer population is embedded in a spherical dark halo that is shallower than the singular isothermal sphere $\left(\gamma_{D M}<2\right)$ in the center, a finite central velocity dispersion $\sigma_{r, 0}$ implies a relation between the central value of the logarithmic slope of the tracers $\gamma_{*, 0}$ and the velocity anisotropy at the center $\beta_{0}$, namely $\gamma_{*, 0}=2 \beta_{0}$. However, it is also possible that the system is dynamically cold at the center (i.e. $\sigma_{r, 0}=0$ ), in which case the condition is $\gamma_{*, 0}>2 \beta_{0}$. This theorem highlights that care is required in the interpretation of results based on assumptions such as isotropy and spherical symmetry.

Ciotti and Morganti (2010) showed that there may be a more general relation that should hold at all radii, which is that $\gamma_{*} \geq 2 \beta$. This may be seen to be related to the positivity of the mass (Eq. 22), as $M \propto \gamma_{*}-2 \beta-\alpha \geq 0$, where $\alpha=d \log \sigma_{r}^{2} / d \log r$. Ciotti and Morganti (2010) have demonstrated the above relation holds for particular forms of the distribution function (namely those in which the augmented density is a separable function of radius and potential, see their Eqs. $(1-6)$ for more details), but the more general inequality (including $\alpha$ ) should be always true.

However, it should also be born in mind that this analysis applies to intrinsic quantities implying for example, that even if $\sigma_{r, 0}=0, \sigma_{\text {los }}$ can still be finite at the center, and hence the theorem is, although correct, less powerful in predicting the orbital behavior at the center. Furthermore, a surface brightness profile might have a very shallow cusp (as considered in Strigari et al., 2010), in which case the velocity ellipsoid need not be isotropic. Another example of the limitation of projected quantities on the power of the theorem is given example below.

Consider the following distribution function

$$
f(E, L) \propto(-E)^{4}\left(-\Phi_{0}-(-E)\right)^{-3} L
$$

where $-\Phi_{0}$ is the potential energy at $r=0$. In this model, the anisotropy is constant, and for this particular example we have fixed it at $\beta=-0.5$. Let 
us now assume that the gravitational potential is of the NFW form:

$$
\Phi(r)=-\Phi_{0} \frac{\log (1+r / a)}{r / a},
$$

here $\Phi_{0}=4 \pi G \rho_{0} a^{3}$, and for simplicity, we have neglected the contribution to the potential by the stars. The light distribution may be obtained by integrating this distribution function with respect to velocity space. The resulting light distribution has a logarithmic slope $\gamma_{*, 0}=0$ at the center as shown in the top panel of Fig. 10. The 2nd moments for the radial and tangential direction are plotted in the central panel of the figure. Note that this model has a centrally vanishing radial velocity dispersion. On the other hand, the l.o.s. velocity dispersion profile is shown in the bottom of the figure, and is relatively flat and non-zero at all projected radii. This distribution function is positive everywhere, it leads to a velocity dispersion profile not too different from that observed for dSphs, but its light distribution at the center has $\gamma_{*, 0}=0$, while the anisotropy is negative $\beta=-0.5$ (so that in this case, $\gamma_{*, 0} \neq 2 \beta_{0}$, but the more general result of Ciotti and Morganti, 2010, does hold, as expected).

\subsection{Modeling through distribution functions}

As stated previously, a solution to the Jeans equation is not necessarily physical since there is no guarantee that a distribution function will exist that is positive definite everywhere. This is one of the reasons why several authors have attempted to model directly the distribution function itself.

Dejonghe \& Merrit (1992) have studied the issue of how the projected velocity distribution as a function of position $f_{\mathrm{los}}\left(v_{\mathrm{los}}, r_{\mathrm{los}}\right)$ for a spherical system constrains the distribution function and gravitational potential. They show that if the form of the spherical potential is specified, then $f(E, L)$ is uniquely determined by $f_{\text {los }}\left(v_{\text {los }}, r_{\text {los }}\right)$. However, if the spherical potential is not known, they argue that there will be a family of possible potentials, but only those that lead to a df that is positive everywhere would be allowed, and not every potential will permit that.

Merritt and Saha (1993) explore the problem of inferring the gravitational potential of a spherical system from measurements of the l.o.s. velocity and positions for individual stars (or galaxies, in their case). They assume that the distribution function may be expressed as a polynomial expansion: $f(E, L)=\sum_{m, n} c_{m, n} f_{m, n}$ where $f_{m, n}=(-E)^{n-1 / 2} L^{2 m}$, hence this distribution function is non-parametric. To determine the properties of the potential, 
however, a few parametric forms are considered. Thus from a discrete set of velocities of galaxies in the Coma cluster, they find best solutions in a maximum likelihood sense. These authors estimate that meaningful constraints are possible with datasets containing $\sim 1000$ objects.

$\mathrm{Wu}$ and Tremaine (2006) (see also Merritt, 1993) take an even more general form for the distribution function, namely they divide the $(E, L)$ space into $N_{E} \times N_{L}$ bins, and construct a set of top-hat basis functions, $h_{m n}=1 / V_{m n}$ where $V_{m n}=\int_{m n} d^{3} x d^{3} v$ is the phase-space volumen associated to bin $m n$. Thus, $f(E, L)=\sum_{m n} w_{m n} h_{m n}$, and the task consists in finding the weights $w_{m n}$ that fit the observables after assuming a specific gravitational potential. Wu and Tremaine (2006) use this technique to infer the mass of M87 from the motions of its globular clusters. These approaches are very powerful as they use maximally the datasets, without turning to moments to characterize the LOSVDs, and are also free of assumptions regarding the distribution function. It would be very valuable to apply such methods to the modeling of dSphs in the Local Group.

Wilkinson et al. (2002) introduce a family of anisotropic distribution functions for spherical systems, in which the dominant gravitational potential is cored and parametrized as $v_{c}^{2}=v_{0}^{2} r^{2} /\left(1+r^{2}\right)^{1+\delta / 2}$. For different values of the characteristic parameters $(-2 \geq \delta \geq 1)$, this leads to flat or declining rotation curves. The velocity ellipsoid is isotropic in the center, and may become radially or tangentially anisotropic at intermediate radii, while at large distances it is constant. The advantage of this family of distribution functions is that the expressions for the various moments (including the 2nd and 4th) are analytic, and depend only on the parameters of the distribution function. This means, that in principle, these characteristic parameters could be retrieved directly through comparison to observations. They also compute the projected (observable) quantities for different values of the parameters. The resulting l.o.s. velocity dispersion profiles (see their Fig. 3) can be flat, rising or declining depending on the distribution function. In Kleyna et al. (2002) they have applied this modeling to a dataset for Draco with $\sim 160$ member stars, and found that the system is best fit by a slightly tangentially anisotropic ellipsoid and with a halo that falls off more slowly than a flat rotation curve model $\left(v_{c} \propto r^{0.17}\right)$, while they are also able to rule out a mass-follows-light model and an extended harmonic core with $3 \sigma$ confidence. 


\subsection{Schwarzschild modeling}

Schwarzschild modeling is by now a traditional technique to derive the mass distribution, especially in elliptical galaxies, from integrated light spectroscopy. It was initially developed in the 1980s (Schwarzschild, 1979; Richstone and Tremaine, 1984), and used extensively to derive $M / L$ and black hole masses in the 1990s and the 2000s (e.g. Rix et al., 1997; van der Marel et al., 1998) where it was extended to allow for axisymmetric models, and even triaxiality (van den Bosch et al., 2008). The basic idea of the method is that the building blocks of galaxies are orbits, and through the right orbital superposition it is possible to match the light and kinematic distributions observed.

Therefore the method consists in assuming a specific gravitational potential, calculating the observables predicted for each orbit, and then weighting the orbits (with non-negative weights) to obtain a model that fits the observed data in a $\chi^{2}$ sense. This approach guarantees that the distribution function obtained (which is reflected in the orbit weights) is non- negative. The fitting procedure thus allows the determination of the characteristic parameters of the best fit model for a specific gravitational potential. If one wishes to test different functional forms for the gravitational potential, then new orbit libraries need to be built, and the fitting procedure is repeated. The advantage of this method is that it does not make assumptions about the form of the anisotropy or the distribution function (rather these are an outcome of the model), and therefore it is less biased than some of the modeling techniques described above. Naturally, it is less flexible in the sense that it is more computationally intensive/expensive, and hence it is possible to explore a smaller variety of gravitational potentials, than for example, through Jeans models.

Despite the vast history, this method has not been applied systematically to the dynamical modeling of dwarf galaxies until very recently. For example, Breddels et al. (2012) have used this technique to model the Scl dSph in the assumption of spherical symmetry. They fit the light, 2 nd and 4 th projected moments. They have found, in agreement with other authors, similar estimates for the mass of Scl within $1 \mathrm{kpc}$, but perhaps more interestingly, and for the first time, they measured the velocity anisotropy of the system to be tangential and relatively flat with radius. Furthermore, they are able to rule out very steep density cusps $\left(\gamma_{D M}>1.5\right)$, although they cannot distinguish statistically an NFW (or shallower cusp) from a $\gamma_{D M}=0$ profile for the dark matter. 
Still considering spherical models, Breddels and Helmi (2013) also fitted the velocity dispersion and kurtosis profiles of Fnx, Sext and Car using this technique. They performed a Bayesian comparison of a suite of different density profiles (2 Einasto, NFW, 4 cored, with different slopes). They found that most models are statistically indistinguishable. However, they show that it is unlikely (with odds of 1:10) that all these dSphs are all embedded in cored profiles where the density falls off steeply as $\rho(r) \propto 1 /\left(1+\left(r / r_{s}\right)^{2}\right)^{\kappa / 2}$, where $\kappa=3,4$. What is also very interesting from their work, is that they show that for each of the systems, the mass distribution from a radius slightly below $\sim r_{1 / 2}$ up to the last measured kinematic data point is the same for all models. This means that even though one cannot reliably distinguish the inner shape or slope of the dark matter halos, they can certainly state what the mass distribution is over a large range of radii, and therefore also derive the slope of the density profile at some intermediate point, as shown in Fig. 11 for the Fnx dSph. Furthermore, since all these models effectively have the same mass distribution, they also have the same anisotropy profile, and hence these authors have essentially measured the anisotropies for these systems. These are found to be relatively flat and mildly tangential $(\beta \gtrsim-0.5)$. This must be telling us something about the formation and evolution of these systems since it must be indicative of some amount of circular motions present in the system (otherwise, for a radial collapse, one would expect a radially anisotropic ellipsoid).

Jardel and Gebhardt (2012) presented three-integral, Schwarzschild models of Fnx that take into account the non-spherical light distribution of this galaxy, although embedded in a spherical dark matter halo. These authors have tested a cored profile $\rho \propto\left(3 r_{c}^{2}+r^{2}\right) /\left(r_{c}^{2}+r^{2}\right)^{2}$ and the NFW model. They find that the cored model is strongly favored, and that the velocity ellipsoid is mildly radially anisotropic. Their mass for $\operatorname{Fnx} M\left(R_{e}\right)=3.9_{-0.11}^{+0.46} \times 10^{7} M_{\odot}$ is somewhat smaller than what the estimators by Wolf et al. (2010) or Walker et al. (2009b, 2010) would predict. Jardel and Gebhardt (2012) argue that this might be related to the fact that those estimators have been established (and shown to be independent of anisotropy) for spherical models. Another difference might lie in that the amplitude of the line-of-sight velocity dispersion profile they derive for Fnx is somewhat lower than that shown, for example, in Fig. 11.

Jardel et al. (2013), return to spherical models, but assume that the density profile for the dark matter is non-parametric. They model Draco in this way, and find that the preferred model is a power-law, with a slope 
quite similar to the NFW, that is $\gamma_{D M}=1$ for $20 \leq r \leq 700 \mathrm{pc}$, and that the velocity ellipsoid is radial. Note that, in comparison to Wilkinson et al. (2002), Jardel et al. (2013) have allowed greater freedom in the form of the density profile (and have not forced cored models), and hence their results are potentially more robust.

\subsection{Made to measure}

The Made-to-Measure (M2M) is a numerical method that integrates the orbits of test particles in a gravitational potential in order to reproduce a given set of observables (Syer and Tremaine, 1996). Particles have associated weights, which themselves follow equations of motion. The system is evolved in time until a satisfactory solution has been found. The gravitational potential may be specified or determined self-consistently, and the resulting distribution function is completely non-parametric, and determined by the final particle's configuration that satisfies the observational constraints. The method can be used to model individual measurements or moments of a LOSVD (as with N-MAGIC in de Lorenzi et al., 2007).

Long and Mao (2010) have modeled Draco using the data from Kleyna et al. (2002) and assumed an isotropic velocity ellipsoid, and the same type of cored potentials as Wilkinson et al. (2002). The best fit model has asymptotic slope for the squared circular velocity $v_{c}^{2}$ of $\delta=-0.90_{-0.35}^{+0.36}$, while for the mass within three core radii they find $9.7 \pm 2.3 \times 10^{7} M_{\odot}$, in comparison to Kleyna et al. (2002) who obtain $\delta \sim-0.34$ and a somewhat smaller mass. Long and $\mathrm{MaO}(2010)$ attribute this difference to their assumption of isotropy.

\subsection{Modeling dSphs with composite stellar components}

As discussed in Sec. 2.3.5 several dSphs host multiple stellar chemodynamical components. Since these components are embedded in the same gravitational potential, they allow one to place more stringent constraints on the properties of this potential, since e.g. each of the component has to satisfy the Jeans equations independently. In practice this means that there are fewer free parameters since each component will follow its own distribution function entering the left-hand-side of Eq. (21), but the right-hand-side will be the same, thereby effectively leading to a reduction in the number of degrees of freedom.

This idea was first exploited by Battaglia et al. (2008a), who modeled Scl using two components, a metal-rich centrally concentrated, and a metal-poor hot and extended, both embedded in a dark matter halo. These authors 
found, using Jeans models, that the metal-poor component was better fit with a nearly flat anisotropy profile, while the metal-rich one, because of its rapidly falling velocity dispersion profile (see Fig. 8), required a radially anisotropic ellipsoid. They found that cored models provided better fits but that NFW models could not be ruled out.

Another use of the composite stellar components was put forward by Walker and Peñarrubia (2011) to infer the slope of the dark matter profile. These authors argue that one might consider Eq. (39) for each component separately, so that the mass of the host halo is constrained at the half-mass radius of each component independently. This then leads to two measurements of the mass at two different radii, and hence to a slope. They have performed many tests of their method, whose basic assumption is that the l.o.s. velocity profiles are flat, and found that their results are relatively robust to such (and other) assumptions, although systematic uncertainties affect the masses at $r_{\text {half }}$ which depend on the density profile of the halo and the degree of embedding of the stars). These authors define

$$
\Gamma=\Delta \log M / \Delta \log r=\frac{\log \left(M_{h, 2} / M_{h, 1}\right)}{\log \left(r_{h, 2} / r_{h, 1}\right)} \sim 1+\frac{\log \left(\sigma_{2}^{2} / \sigma_{1}^{2}\right)}{\log \left(r_{h, 2} / r_{h, 1}\right)},
$$

where $r_{h \text {,pop }}$ and $M_{h \text {,pop }}$ refer to the projected half-light radius and the mass at this point, while $\sigma_{\text {pop }}^{2}$ is the global velocity dispersion that characterizes the population, and where pop $=1,2$, i.e. metal-rich or metal-poor components. In the limit of $r \rightarrow 0$, then $d \log M / d \log r=3-\gamma_{D M}$ where $\gamma_{D M}$ is the central value of the slope of the dark matter density profile. Since $d \log M / d \log r$ decreases as $r$ increases for any reasonable density profile, this implies that $3-\gamma_{D M}>\Gamma$, or alternatively that $\gamma_{D M}<3-\Gamma$, as the slope $\Gamma$ is measured at a finite distance from the center. Walker and Peñarrubia (2011) find $\Gamma=2.61_{-0.37}^{+0.43}$ for Fnx, while for Scl $\Gamma=2.95_{-0.39}^{+0.51}$. This thus implies that NFW-like profiles $\left(\gamma_{D M}=1\right)$ would be ruled out at significance levels $\gtrsim 96 \%$ and $\gtrsim 99 \%$ respectively for these systems. These results are much more stringent than any of the previously reported findings by other authors, where typically both profiles are consistent with the data.

More recently, Amorisco and Evans (2012b) have modeled the two populations in Scl using Michie-King models. These are isotropic in the center and become radially anisotropic in the outskirts. The validity of these assumptions for the velocity ellipsoid is taken from their analysis of the shape of the l.o.s. velocity distributions of Scl in Amorisco and Evans (2012c), whose estimates of the 4th moment would suggest a radially anisotropic ellipsoid 
(see however Breddels et al., 2012, who find a kurtosis profile that is consistent with tangential anisotropy. As discussed in Sec. 2.3.4 the difference is possibly related to the treatment of foreground/membership determination). Under these assumptions for the velocity ellipsoid (or the distribution function) these authors find that cored mass distributions are preferred over cusped ones. Given the uncertainties in the measurements of the 4th moments, this result could be related to the assumed shape of $\beta(r)$ rather than necessarily reflect the underlying mass distribution.

Agnello and Evans (2012) use the projected virial theorem and argue that the two populations in Scl should satisfy independently the virial theorem,

$$
\frac{K_{l o s, 1}}{K_{l o s, 2}}=\frac{W_{l o s, 1}}{W_{l o s, 2}}
$$

from which they obtain the relation

$$
\left(\frac{\sigma_{0,1}}{\sigma_{0,2}}\right)^{2}>2\left(\frac{R_{h, 1}}{R_{h, 2}}\right),
$$

if the stars follow Plummer profiles and are embedded in NFW halos. Given their estimates of these various observables, Agnello and Evans (2012) conclude that no NFW halo can be compatible with the energetics of the two populations. Because the two populations should co-exist in virial equilibrium, the authors argue that this implies that the dark halo must be cored, and they estimate its size to be $\sim 120 \mathrm{pc}$.

The results presented in this section all argue that the modeling using two (or multiple) components disfavor NFW/cuspy profiles for dSphs, at least for Fnx and Scl. It is striking that the consideration of two components in dynamical equilibrium point all in the same direction. It would be important to confirm these results using fully-fleshed non-parametric methods, such as Schwarzschild or Made-to-Measure, that explore the presence of multiple populations and remove some of the (systematic) uncertainties in the use of global scaling relations. It would also be desirable to understand the extent down to which these systems' properties are better described using a few independent components, rather than to assume that the properties of the stars change gradually throughout the system, and specifically how these assumptions affect the dynamical models and their conclusions.

Figure 12 compares the results of various modeling approaches on Scl. In this figure we have plotted the mass distribution derived using Schwarzschild 
models by Breddels and Helmi (2013). We have included here estimates of $M_{300}$ by Strigari et al. (2008) and Walker et al. (2009b) and at the 3D half-light radius by Wolf et al. (2010). These estimates are all consistent with those obtained by Breddels and Helmi (2013) which is reassuring. The mass estimated at $1.8 \mathrm{kpc}$ obtained by Battaglia et al. (2008a) assuming a cored density profile but modeling simultaneously metal-rich and metal-poor populations is also shown (open black circle). It is on the upper side of the curves, but is consistent within error bars, and is beyond the region where the mass profiles are indistinguishable, so this mass estimate is likely to be more model dependent. Finally, the two estimates of the mass derived by Walker and Peñarrubia $(2011)$ are shown as diamonds in this Figure. These two estimates of the mass at the projected half-light radius of the metal-rich and metal-poor components of Scl, appear to be somewhat larger than what is found by Breddels and Helmi (2013). This is consistent with the systematic uncertainties that Walker and Peñarrubia (2011) reported from their Monte Carlo simulations. However, we notice that the mass at the half-light radius of the metal-poor component is more overestimated than that of the metalrich one (and even higher than $M_{300}$ or $M_{1 / 2}$ for example). In view of this, it seems plausible that the slope $\Gamma$ that Walker and Peñarrubia (2011) derived could be overestimated, in which case, cuspy profiles with $\gamma_{D M}>0$ could still be allowed.

\section{Future directions}

In the previous sections we have discussed the status of the field, and have begun to identify directions where more research would be desirable to understand the properties and dynamics of dSphs. In the case of the dynamical modeling, as this review reflects, much of this work has been done assuming that the dSphs are embedded in spherical dark matter halos (and often, even assuming their light distribution is approximately spherical). First attempts to veer from this assumption have been made using the Jeans equations, but as discussed earlier, these have the limitation of exploring parametric models, and unfortunately, the results sometimes reflect the hypotheses made. It is therefore desirable to apply non-parametric modeling, for example along the lines of Jardel and Gebhardt (2012, although these authors still assume the dark halos are spherical), who have used Schwarzschild models assuming non-spherical light distributions. In fact, van den Bosch et al. (2008) have performed triaxial modeling of elliptical galaxies, implying that the tools 
needed for the dSphs may already have been largely developed (see also van de Ven et al., 2006, for the modeling of OmegaCen in the limit of axisymmetry). Another example, is the M2M modeling of the Galactic bar by Long et al. (2013). Unfortunately the sample sizes for the most classical dwarfs may be still too small to warrant such sophisticated approaches, with the possible exceptions of Sculptor and Fornax. Nonetheless, it is worthwhile establishing what are the degeneracies/limitations in the modeling, and to what extent they can be broken by different datasets. This can be addressed by applying dynamical models on Mock datasets, for example extracted from N-body simulations or generated from known distribution functions.

Another aspect is the consideration of the discrete nature of the datasets, which has not been exploited in full. Most works so far fit the full LOSVD or its moments, both as function of projected radius $R$, rather than attempting a full likelihood analysis using the individual measurements (position on the sky, and l.o.s. velocity) for each star in the dataset. This is a direction that needs to be exploited further, since binning always leads to loss of information. First steps have been taken in the context of Schwarzschild models by Chanamé et al. (2008), or when using distribution functions to model the dynamics of planetary nebulae or globular cluster systems around elliptical galaxies by Merritt and Saha (1993) and Wu and Tremaine (2006). There is also recent work employing the Jeans equations for modeling the dynamics of galaxies in a cluster environment (Mamon et al., 2013).

The use of proper motion measurements of stars in dSphs is another unexplored aspect of the dynamical modeling. The reason is, of course, that this has been beyond the capabilities of current instrumentation. However, the situation is likely to change in the coming years. For example, it is now possible to constrain the mean tangential motions of dSphs using the Hubble Space Telescope (see Piatek and Pryor, 2008, and references therein), and these measurements are likely to be significantly more accurate with the advent of Gaia 8 . The internal motions may still be just about beyond reach for individual stars in dSphs. For example for a star at $70 \mathrm{kpc}$, an internal tangential velocity of $v_{t} \sim 10 \mathrm{~km} \mathrm{~s}^{-1}$ translates into proper motion of $\mu \sim 30 \mu \mathrm{as} / \mathrm{yr}$. For a star of magnitude $G \sim 17$, the accuracy expected for the Gaia mission is $\sigma_{\mu} \sim 36 \mu \mathrm{as} / \mathrm{yr}$, and hence the internal velocity and

\footnotetext{
${ }^{8}$ See http:www.rssd.esa.intindex.php?project=GAIA\&page=Science_Performance for the latest estimates of its performance.
} 
its error will be of comparable magnitude. This implies, however, that one ought to be able to bin the data to obtain a tangential velocity curves with reasonable accuracy, as the error on the dispersion is inversely proportional to the square root of the sample size. For the UFDs the situation is less clear, as these objects have faint and sparsely populated red giant branches. At these characteristic faint magnitudes Gaia's proper motion accuracies degrade quickly, from $\sim 80 \mu \mathrm{as} / \mathrm{yr}$ at $G=18$, to $140 \mu \mathrm{as} / \mathrm{yr}$ at $G=20$. For an object at a distance of $\sim 40 \mathrm{kpc}$, this implies an error in the tangential velocity of $\sim 15-25 \mathrm{~km} \mathrm{~s}^{-1}$. Although in principle one can reduce this error by binning, the sparsely populated RGBs prevent from obtaining the significant gains needed to characterize the internal kinematics of the UFDs. Nonetheless, these measurements clearly will allow the determination of their orbit, as well as aid in establishing membership and potentially finding extratidal stars and streamers. On the other hand, for the brightest stars in the LMC (those with $G \lesssim 15$ ), the expected accuracies are in the range $\sim 4-14 \mu \mathrm{as} / \mathrm{yr}$, which at a distance of $\sim 50 \mathrm{kpc}$, corresponds to a tangential velocity error of $\sim 0.1-3.3 \mathrm{~km} / \mathrm{s}$, comparable to what can be obtained nowadays for the l.o.s. velocities routinely from the ground.

Wilkinson et al. (2002) have studied the impact of proper motion information following the specifications planned for the former SIM mission, namely $3-6 \mu \mathrm{as} / \mathrm{yr}$, which translates into $1-2 \mathrm{~km} / \mathrm{s}$ for stars in Draco of magnitude $V \sim 19-20$. These authors show that by adding proper motion information for samples as small as 160 stars, it is possible to obtain accurate estimations of both the velocity anisotropy and mass slope, and thereby break modeling degeneracies unambiguously. This is also confirmed by Strigari et al. (2007a), who show that, for general dark matter density and anisotropy profiles, the log slope of the dark matter profile at about $\sim 2 r_{c}$ can be measured to within \pm 0.2 if the proper motions of 200 stars (with tangential velocity errors of $\sim 5$ $\mathrm{km} / \mathrm{s}$ ) are added to the l.o.s. velocity measurements. This would allow to place tighter constraints on the type of dark matter halos hosting dSphs, and hence possibly also on the nature of dark matter.

Dynamical modeling of dwarf galaxies is not only interesting to understand the mass distribution of these systems, but it is also important in terms of evolutionary paths. Numerical simulations of isolated dwarf galaxies (e.g. Valcke et al., 2008; Revaz et al., 2009) model these systems as the result of the radial collapse of a gas cloud embedded in a dark matter halo. As a consequence of the collapse, the orbital distribution is likely biased to radial orbits. On the other hand, if there is any amount of angular momentum, as 
expected in the context of $\Lambda \mathrm{CDM}$, the gas might collapse towards a disk-like distribution (see Governato et al., 2010; Sawala et al., 2010; Schroyen et al., 2011), but this might depend on the amount of merger activity at the time of formation of most of the stars (Gnedin and Kravtsov, 2006). The spheroidallike morphologies might thus have to be the result of external mechanisms, and it has been proposed by Mayer et al. (2001) that the tidal force field of the host galaxy (in this case, the Milky Way) can be the driver of morphological transformations. These authors, and more recently, Kazantzidis et al. (2013) have shown, that the dSphs could be tidally stirred disky dwarf galaxies. The morphologies, because of the stirring, would become triaxial, and the systems could lose most of their angular momentum and end up being largely pressure supported, with nearly isotropic velocity ellipsoids (Łokas et al., 2010). Helmi et al. (2012) have proposed another scenario to transform disk-like dwarf galaxies into spheroidal systems through a major merger (with a dark satellite) (see also Kazantzidis et al., 2011b, for a merger between dwarfs). While such events are not necessarily very common, they might lead to a spheroidal morphology. In either case, one might expect the orbital distribution to be biased to high-angular momentum orbits as a reflection of the initial conditions.

Hydrodynamical simulations are also important to understand the interplay between baryons and dark matter, and to place firmer predictions on the expected properties of dark matter halos, such as their shape and density profile (Di Cintio et al., 2012; Zolotov et al., 2012). Baryonic processes such as feedback are poorly understood, but it is on the scale of dwarf galaxies that we are likely to obtain the largest insights because of the shallowness of their potential wells, and also because these systems are the easiest to resolve numerically.

The dynamics of the dwarf galaxies in the Local Group are also of interest, as it has been proposed that they may be tidal dwarf systems, and in that case, expected to be devoid of dark matter. This proposal has been put forward to explain the somewhat peculiar relatively planar distribution of satellites around the Milky Way (Metz et al., 2009). More recently, Ibata et al. (2013) has even shown that about half of the satellites of M31 appear to be located in thin vast co-rotating plane, which Hammer et al. (2013) explain in the context of a gas-rich merger experienced by M31 5 Gyr ago. However, it is unclear whether the internal dynamics of the dSphs, their extended star formation histories and complex chemical enrichment evolutionary paths, and the fact that they have survived until the present 
day would support such a scenario. Therefore it is important to do a detailed comparison between the simulations and the rich datasets that are already available. Clearly, the impact of the Gaia mission will also be enormous here because thanks to the accurate proper motion measurements it will possible to reconstruct the full orbital history of the satellites around the Milky Way.

The Milky Way system of satellite galaxies is likely to remain the place where we will be able to gather the most accurate kinematic data-sets for resolved stars. However it would be desirable to expand the sample of dwarf galaxies to explore different environments. For isolated dwarf galaxies, we can exclude environmental effects such as tidal and ram-pressure stripping as major drivers of their present stellar and DM properties. On the other hand, probing the satellite system of other large spirals such as M 31 would highlight possible differences due to mass of the host and assembly history of the whole system, and provide larger statistics.

There are some works that have started to gather large samples of RGB stars for kinematic studies in Local Group dwarf galaxies, beyond the MW system (e.g. the isolated WLM at $\sim 1 \mathrm{Mpc}$ observed both with VLT/FORS and Keck/DEIMOS Leaman et al., 2012), although this implies a significant investment of telescope time per galaxy. For the closest ones (for example, the M 31 satellites found on the close side to the MW), if populous enough, it is possible to gather kinematic samples of comparable size and quality as those for MW dSphs (e.g. as for And II, observed with Keck/DEIMOS Ho et al., 2012), suitable for a full dynamical modeling. For the most remote and intrinsically faint Local Group dwarfs, it is already challenging to gather samples of a few dozens l.o.s. velocities (e.g. VV124 Kirby et al., 2012), which permits to apply the scaling relations discussed in Sect. 3. The challenge can be easily understood if we consider that the tip of the RGB at the distance of $\mathrm{VV} 124(1.1 \mathrm{Mpc})$ is at about $I \sim 21.2$, about 5.5mag fainter than for the typical dSph at $\sim 80 \mathrm{kpc}$. The situation will be greatly improved by the next generation of $30 \mathrm{~m}-40 \mathrm{~m}$ class telescopes, in particular if equipped with spectrographs with multiplex capabilities over a field-of-view of one to a few arcmin to encompass a large enough portion of these galaxies. This would also allow targetting much fainter stars in the MW UFDs, and significantly expanding their kinematical datasets. Accurate l.o.s. velocities of large samples of evolved red giant stars would become a reality even up to 4 Mpc (e.g. Evans et al., 2013, and E-ELT Design Reference Mission re- 
port ${ }^{9}$, opening the exploration of the satellite systems of other large spirals such those in the Sculptor group.

\section{Conclusions}

In the last decade, we have experienced the vast increase in the number and extent of datasets with kinematic measurements of stars in the dSphs of the MW.

The leap forward in sample size and spatial coverage, coupled to exquisite velocity accuracy, due to advent of wide-field multi-object spectrographs on the largest telescopes world-wide allowed us to uncover velocity gradients of a few $\mathrm{km} \mathrm{s}^{-1} \mathrm{deg}^{-1}$ (e.g. Muñoz et al., 2006; Battaglia et al., 2008a; Walker et al., 2008; Ho et al., 2012) and to establish that the classical dSphs have nearly flat $\sigma_{\text {l.o.s }}(R)$ around $6-10 \mathrm{~km} \mathrm{~s}^{-1}$. In combination with the metallicity information, this has unveiled an unexpected degree of complexity in these small galaxies, such as the presence of multiple stellar chemo-dynamical components (Tolstoy et al., 2004; Battaglia et al., 2008a, 2011).

These superb datasets have been modeled using a variety of techniques, mostly assuming spherical symmetry. They have confirmed that dSphs are the most dark matter dominated objects known to date, with $M / L \sim 10-$ 1000 in the regions where the stars are located. Their masses at their half-light radii have been accurately measured, and are in the range of $\sim 4 \times 10^{5} \mathrm{M}_{\odot}$ for Wilman I to $7.5 \times 10^{7} \mathrm{M}_{\odot}$ for Fnx (Wolf et al., 2010; Walker et al., 2009b, 2010). These estimates are robust as they do not suffer from uncertainties in the anisotropy of the velocity ellipsoid. Thanks to sophisticated modeling techniques such as Schwarzschild's method, we have begun to discover that the internal motions of dSphs do not deviate strongly from being isotropic, with a slight preference for constant slightly tangentially anisotropic ellipsoids for some of the dSphs (Breddels and Helmi, 2013). This would be in contrast to what has recently been found for some round giant ellipticals, which have radially aligned ellipsoids (e.g. de Lorenzi et al., 2009; Morganti et al., 2013). This would suggest that the dwarf and such giant spheroids could have had different formation paths.

Although the debate concerning whether the central density profile is cusped or cored still remains for these systems, we would like to argue that

\footnotetext{
${ }^{9}$ http://www.eso.org/sci/facilities/eelt/science/drm/drm_report.pdf
} 
this dichotomy is passé, and that the exact value of the slope might depend on the specific history of the baryons in these systems. Furthermore, from an observational perspective, it is very hard to obtain the kinematics of stars truly located near the center of the dSph and not just in projection, to use these to constrain the slope. Nevertheless, we have made substantial progress in demonstrating that it is possible to measure robustly (in a model independent fashion) the shape of the mass distribution $M(r)$ over a distance range of $\sim 1 \mathrm{kpc}$ in projection (Breddels and Helmi, 2013). These measurements are likely to be more constraining for cosmological simulations that the measurement of the very central slope.

The next years promise to be exciting in the field because of advances both on the theory as well as the observational front. Theoretically, this will be driven by more sophisticated dynamical modeling techniques, and by the large improvements in numerical simulations of galaxy formation on the dwarf galaxies scale. From an observational perspective, larger radial velocity surveys, and the advent of proper motion information should enable us to understand the dynamics and evolution of the dSphs in much better depth.

\section{Acknowledgments}

G. Battaglia gratefully acknowledges support through a Marie-Curie action Intra European Fellowship, funded from the European Union Seventh Framework Program (FP7/2007-2013) under grant agreement number PIEFGA-2010-274151. A. Helmi and M.A. Breddels acknowledge financial support from NOVA (the Netherlands Research School for Astronomy), and the European Research Council under ERC-StG grant GALACTICA-24027. We are also grateful to numerous enjoyable interactions throughout the past few years with members of the GALACTICA group: H. Tian, T. Starkenburg, H. Buist, G. Monari, C. Vera-Ciro, E. Starkenburg, T. Antoja, R. Sanderson, S. Jin and K. Westfall. Finally, we would like to thank M. Cappellari for his generosity in making Fig. 9. We acknowledge discussions with S. Koposov, E. Lokas, J. Peñarrubia, and M. Walker. 


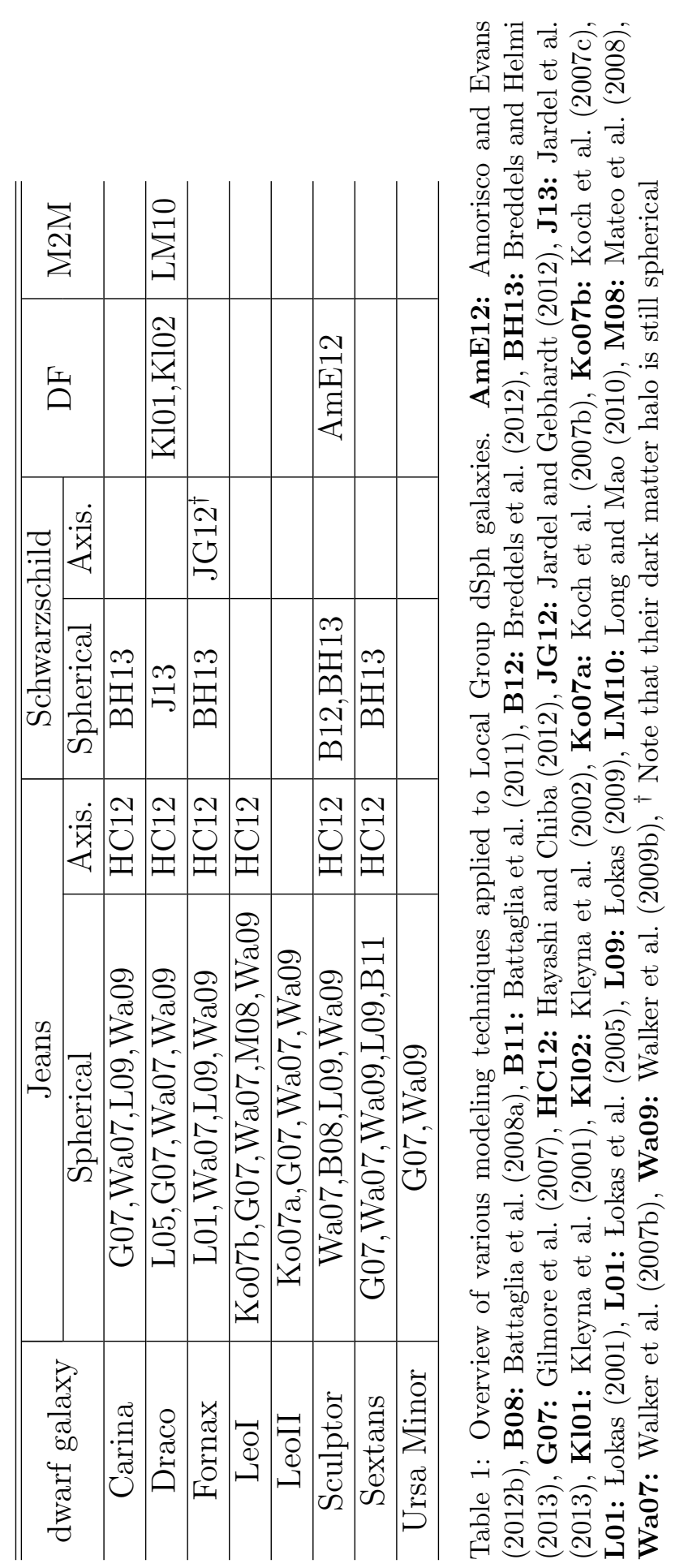




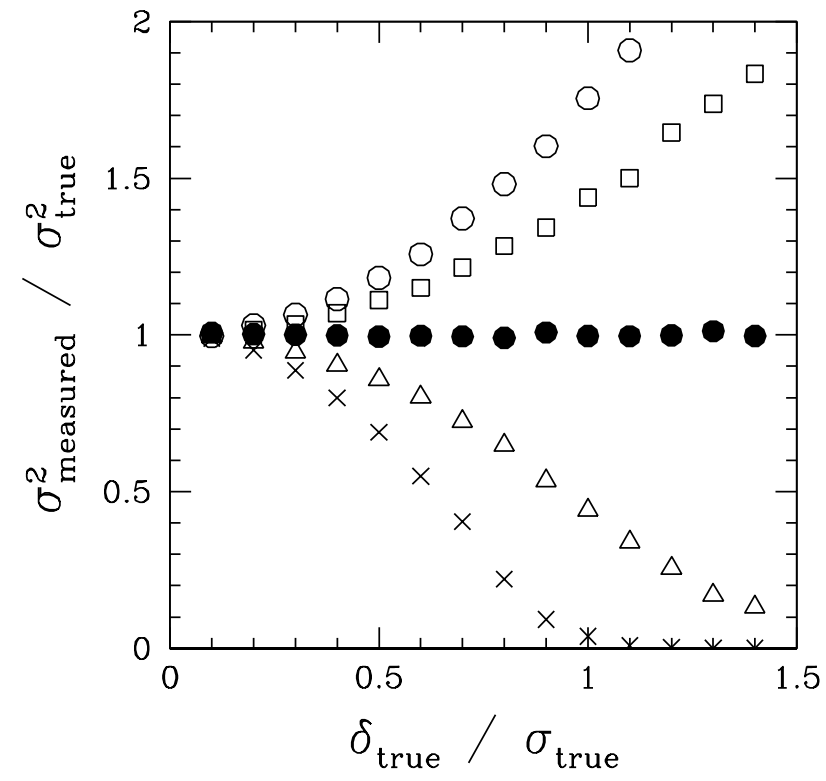

Figure 1: This figure (from Koposov et al., 2011, reproduced by permission of the American Astronomical Society) illustrates the accuracy of velocity dispersion estimates, expressed as the ratio of the measured variance over the true one, as a function of the ratio of velocity error $\delta_{\text {true }}$ to the true velocity dispersion $\sigma_{\text {true }}$. The filled circles show the case of perfectly known velocity errors; open squares and circles when the errors used in the analysis are underestimated by a factor of 0.5 and 0.75 ; the triangles and the crosses for an overestimation of 1.25 and 1.5 , respectively. 


\section{References}

Aaronson, M., Mar. 1983. Accurate radial velocities for carbon stars in Draco and Ursa Minor - The first hint of a dwarf spheroidal mass-to-light ratio. ApJL 266, L11-L15.

Aaronson, M., Olszewski, E., 1987. The search for dark matter in Draco and Ursa Minor - A three year progress report. In: Kormendy, J., Knapp, G. R. (Eds.), Dark matter in the universe. Vol. 117 of IAU Symposium. pp. 153-158.

Adén, D., Feltzing, S., Koch, A., Wilkinson, M. I., Grebel, E. K., Lundström, I., Gilmore, G. F., Zucker, D. B., Belokurov, V., Evans, N. W., Faria, D., Nov. 2009a. A photometric and spectroscopic study of the new dwarf spheroidal galaxy in Hercules. Metallicity, velocities, and a clean list of RGB members. A\&A 506, 1147-1168.

Agnello, A., Evans, N. W., Aug. 2012. A Virial Core in the Sculptor Dwarf Spheroidal Galaxy. ApJL 754, L39.

Amorisco, N. C., Evans, N. W., Sep. 2012a. A Troublesome Past: Chemodynamics of the Fornax Dwarf Spheroidal. ApJL 756, L2.

Amorisco, N. C., Evans, N. W., Jan. 2012b. Dark matter cores and cusps: the case of multiple stellar populations in dwarf spheroidals. MNRAS 419, $184-196$.

Amorisco, N. C., Evans, N. W., Aug. 2012c. Line profiles from discrete kinematic data. MNRAS 424, 1899-1913.

An, J. H., Evans, N. W., May 2006. A Cusp Slope-Central Anisotropy Theorem. ApJ 642, 752-758.

An, J. H., Evans, N. W., Aug. 2009. A Theorem on Central Velocity Dispersions. ApJ 701, 1500-1505. 

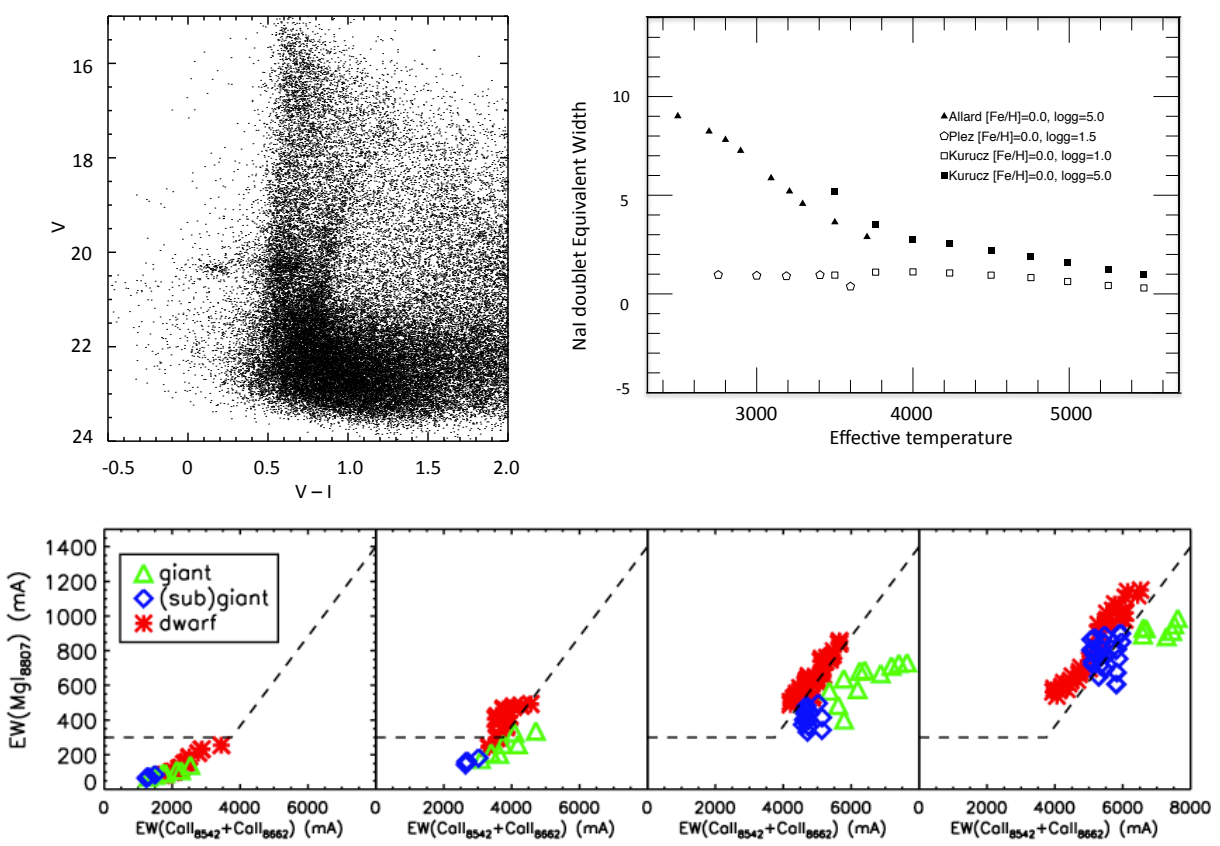

Figure 2: Top left: Color-magnitude diagram of stellar objects along the l.o.s. to the Sextans dSph from INT/WFC and ESO/WFI data (see Battaglia et al., 2011) showing how heavily contaminated by MW foreground stars is red giant branch of this dSph. Top right: Equivalent width of the $\mathrm{Na}$ I feature, for giant stars (open symbols) and dwarf stars (filled symbols) from Schiavon et al. (1997, reproduced by permission of the American Astronomical Society). Bottom: Summed EW of the two strongest CaT line versus the MgI8806.8 line. Giants above the horizontal branch are shown as green triangles, (sub)giants below the horizontal branch as blue diamonds and dwarfs as red asterisks (for definition see Battaglia and Starkenburg, 2012). Overplotted in each panel is the line separating dwarf and giant stars as given by Eq. (4). Figure from Battaglia \& Starkenburg 2012, A\&A, 539, 123, reproduced with permission (C)ESO. 

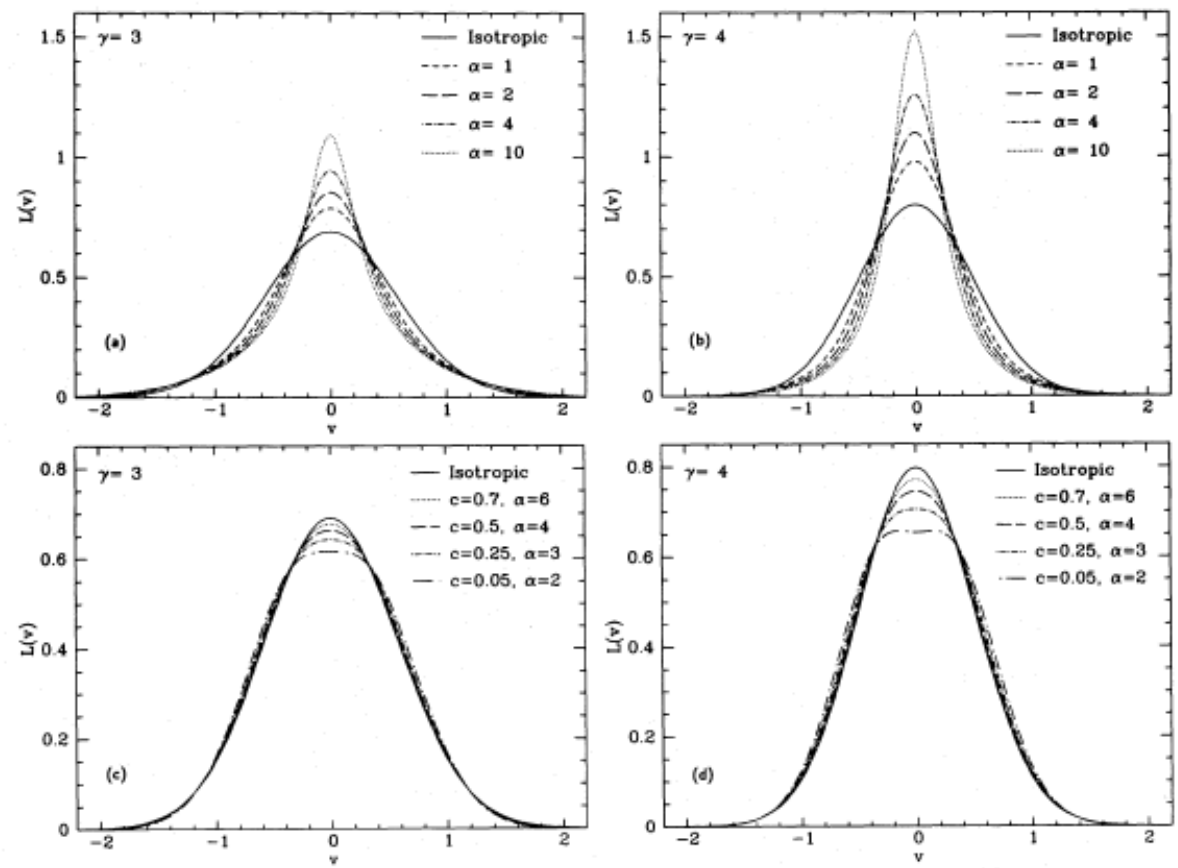

Figure 3: L.o.s. velocity profiles for quasi-separable distribution functions in the potential of a singular isothermal sphere from Gerhard 1993, MNRAS, 265, 213, reproduced by permission of Oxford University Press. Radially anisotropic and tangentially anisotropic models are shown in the top and bottom panels, respectively. The stellar density is $\rho \propto r^{-3}$ (left) and $\rho \propto r^{-4}$ (right). See Gerhard (1993) for more details. 


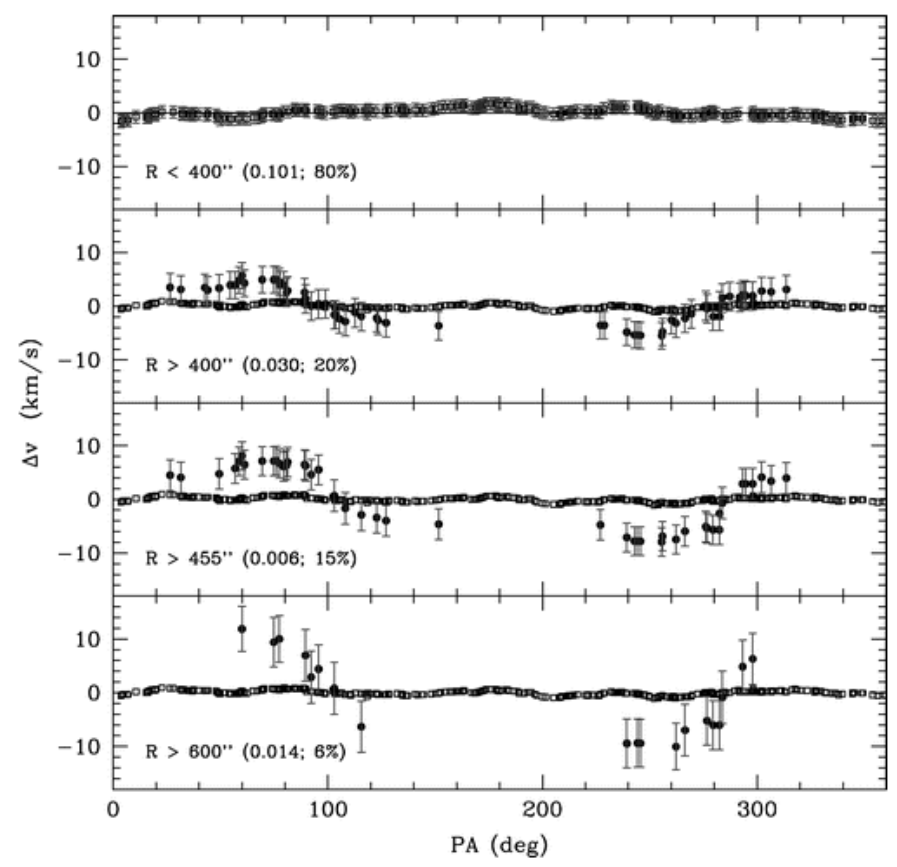

Figure 4: Difference of mean velocities of Leo I members on both sides of a bisector oriented along a given position angle (P.A.) from Mateo et al. (2008, reproduced by permission of the American Astronomical Society). The labels indicate the radial range of the data-set plotted in each panel (points with error-bars), the probability of exceeding $\delta v_{\max }$ and the fraction of Leo I members used to produce each plot. In the panels with $R>400$ ", the symbols without error bars are the data-set from the full sample $(\mathrm{N}=328)$. 

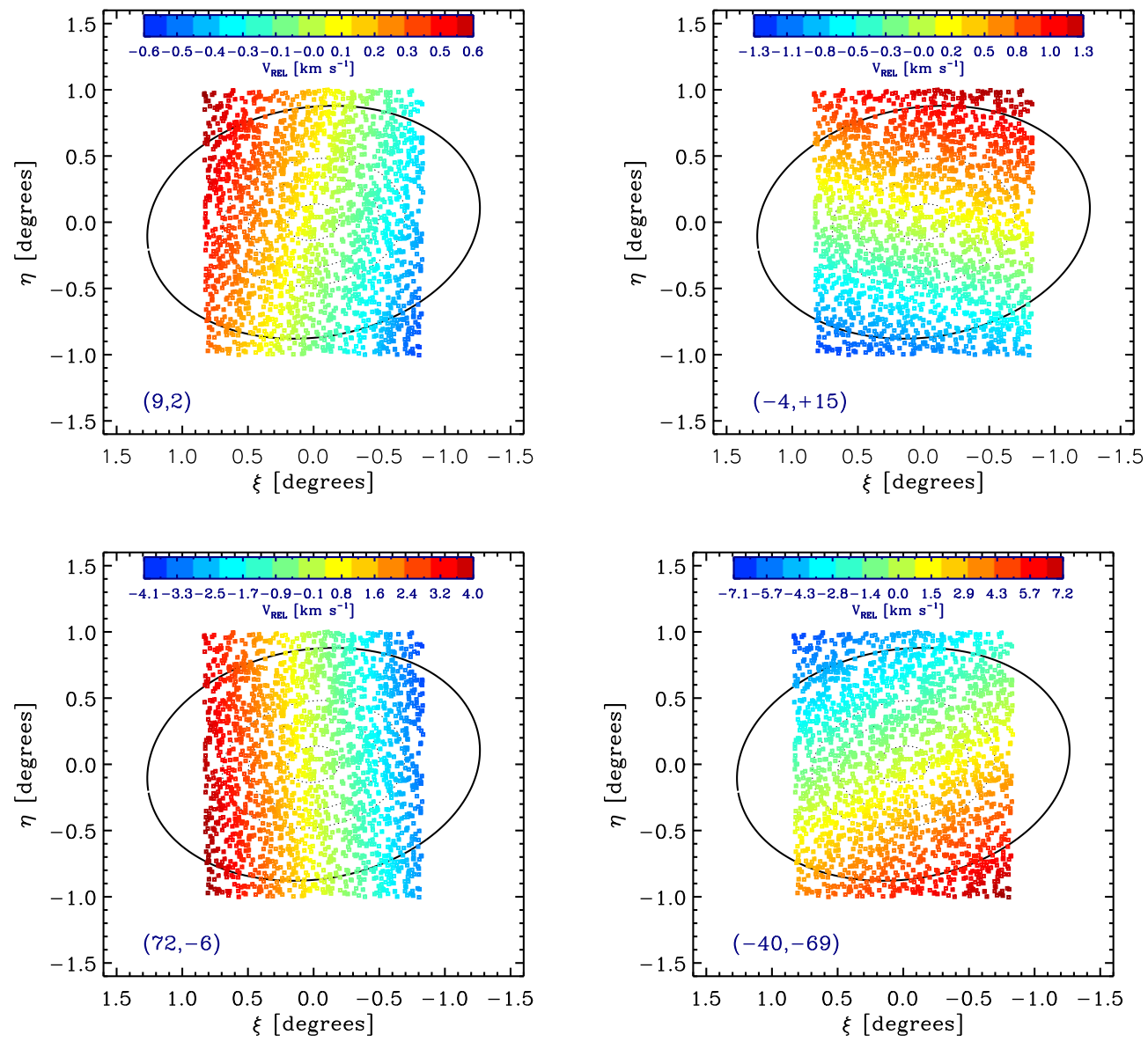

Figure 5: Velocity field of the projection of the relative motion between the Sun and the Sculptor dSph along the line of sight defined by equatorial coordinates $(\alpha, \delta)$. The $v_{r m r e l}(\alpha, \delta)$ has been derived applying the formulae in the Appendix of Walker et al. (2008) and subtracting Sculptor systemic heliocentric velocity. The coordinates of the mock data-set (squares) were randomly extracted from a uniform distribution in $\alpha$ and $\delta$ of $\pm 2 \mathrm{deg}$ around Sculptor's center. The four panels assume different values for the Sculptor proper motion $\left(\mu_{\alpha}, \mu_{\delta}\right)$ [mas/century] in the heliocentric reference frame, as indicated by the labels: top left: from Piatek et al. (2006); top right: value from Piatek et al. (2006), but taking $\left(\mu_{\alpha}-1 \sigma, \mu_{\delta}+1 \sigma\right)$; bottom left: from Schweitzer et al. (1995); bottom right: from Walker et al. (2008). 

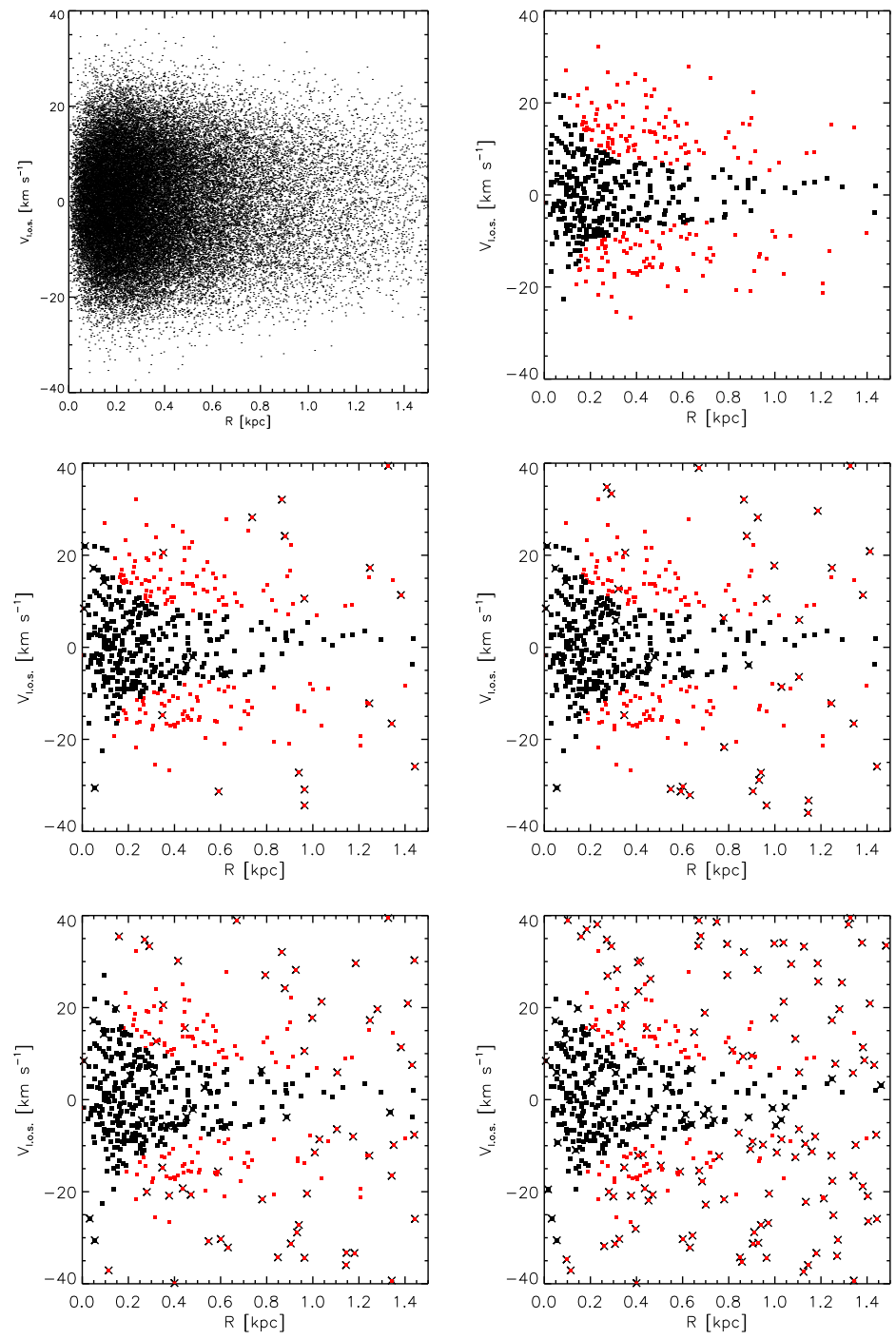

Figure 6: Top left: L.o.s. velocity versus projected radius $R$ diagram for a mock Sculptor galaxy (Breddels et al. 2012) (50000 points). Top right: application of the virial theorem interloper rejection procedure applied to a randomly extracted sub-sample of 500 stars from the mock Sculptor (see text). The red squares show the objects rejected after three iterations and the black squares those retained in the sample. Middle and bottom panel: as in the previous panel, but adding an increasing amount of mock MW interlopers, shown by the crosses (see text). The interloper rejection procedure correctly identifies the mock MW interlopers found outside of the velocity envelope, but also rejects an important fraction of genuine members: initially, the genuine members are $95 \%, 91 \%, 85 \%$ and $70 \%$ of the sample of 500 objects, while after the 3rd iteration only the $60-65 \%$ are accepted as members (therefore the remaining $32 \%, 27 \%, 21 \%$ and $13 \%$ are mistaken for interlopers). 

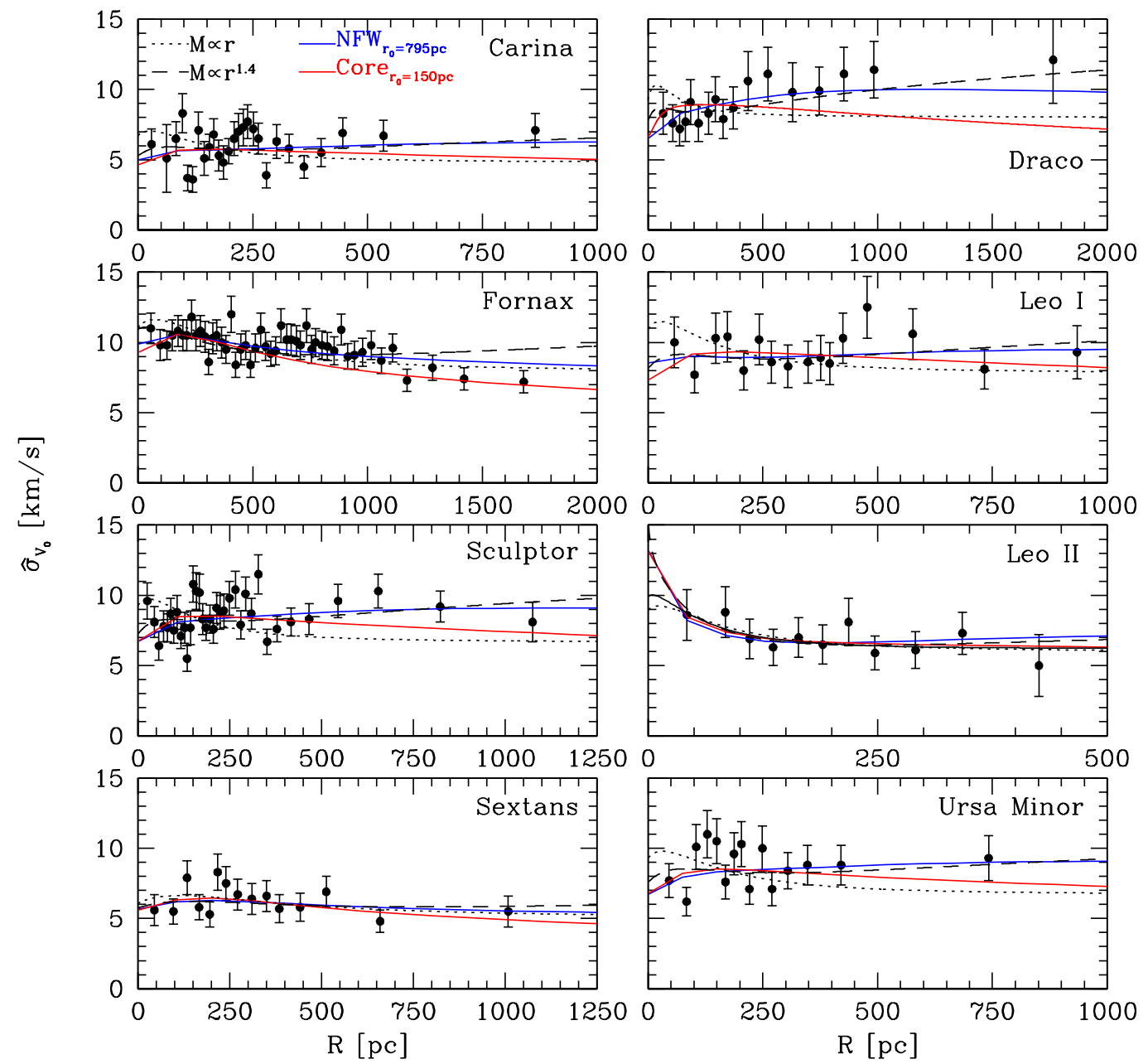

Figure 7: Projected velocity dispersion profiles for eight bright dSphs obtained by Walker et al. (2009b reproduced by permission of the American Astronomical Society). The profiles calculated from isothermal, power-law, NFW and cored halos (with $M(r) \propto r^{1.4}$ are also shown. These fits have been obtained using the spherical Jeans equations, and in particular Eqs. 23) and (31). For each type of halo these authors fit only for the anisotropy (assumed to be constant) and mass normalization (at a given scale/distance). The scale radius of the system is fixed for the various models as indicated in the panels. 


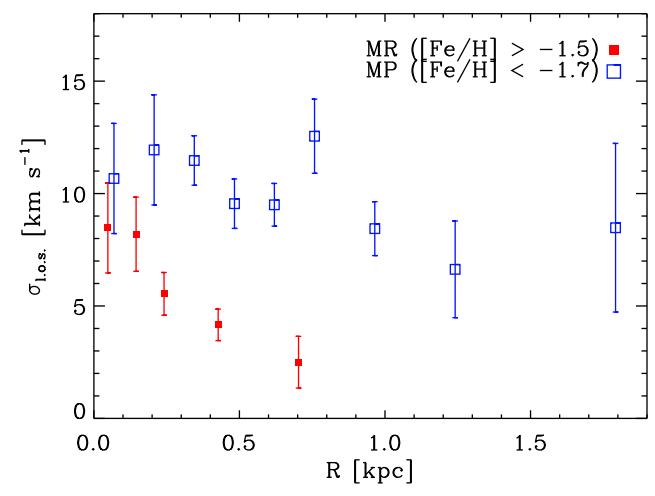

Figure 8: L.o.s. velocity dispersion profiles for the Sculptor members more metal-rich than $[\mathrm{Fe} / \mathrm{H}]=-1.5$ (filled squares) and more metal-poor than $[\mathrm{Fe} / \mathrm{H}]=-1.7$ (open squares), from rotation-subtracted velocities in the Galactocentric Standard of Rest system (see Battaglia et al. 2008a). 


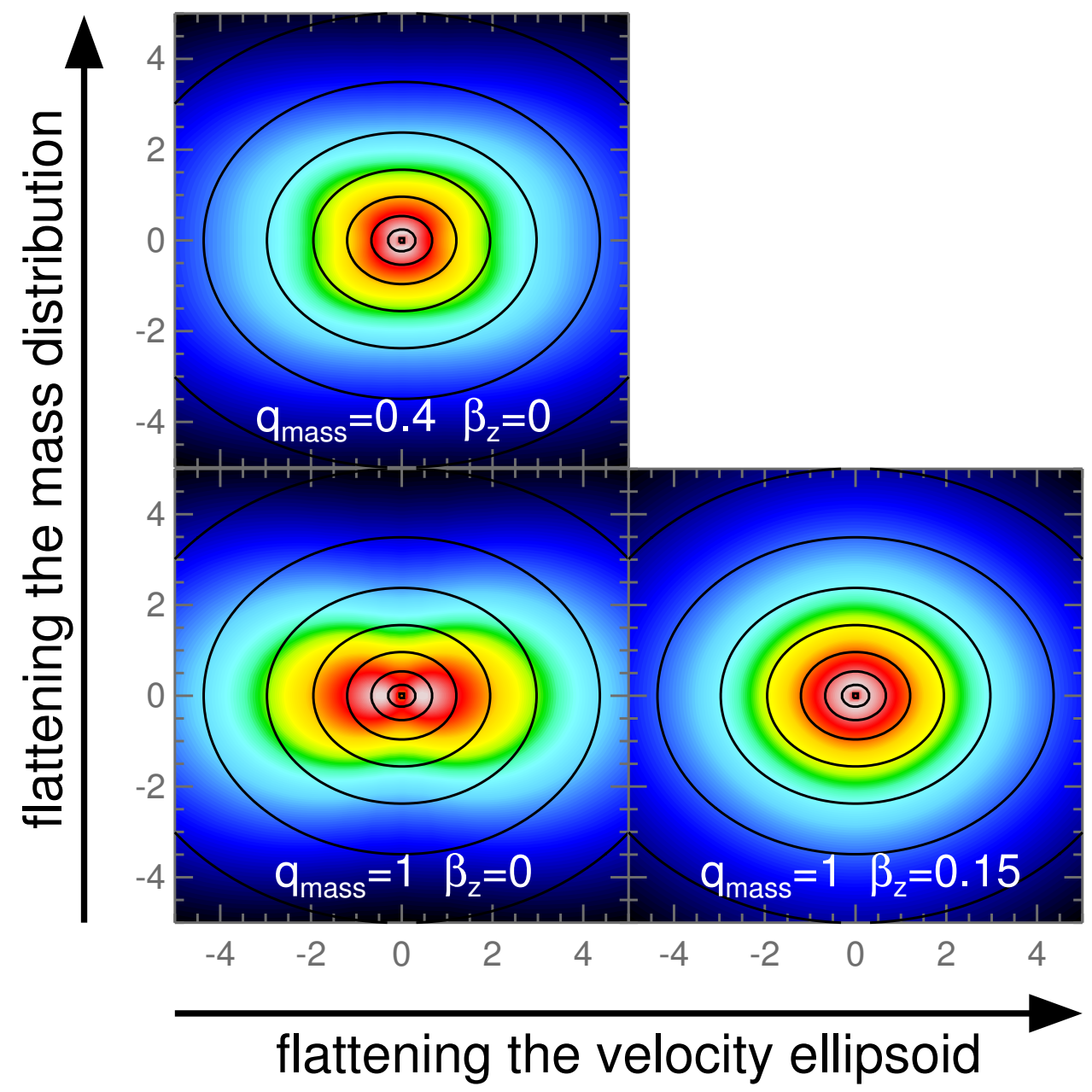

Figure 9: JAM models by Cappellari (2008) of Hernquist (1990) profiles for both the light and mass distribution, where for simplicity the stars have been treated as a massless tracers in the given potential. Plotted is the true second moment $V_{R M S}=\left\langle v_{l o s}^{2}>^{1 / 2}=\right.$ $\sqrt{V^{2}+\sigma^{2}}$ of the models as given in Eq. (28) of Cappellari (2008). The spherical Hernquist mass distribution was flattened while keeping the surface density profile unchanged along circularized isophotes $r=\sqrt{a b}$. By definition $\beta_{z}=1-\sigma_{z}^{2} / \sigma_{R}^{2}$. In all cases the light distribution was kept fixed with $q=0.8$, as shown by the isophotes (in 0.5 mag steps). The color scale in the plots is not the same, but it is adapted to the ranges of values in each panel. The axis are in units of the Hernquist scale radius $a$. 

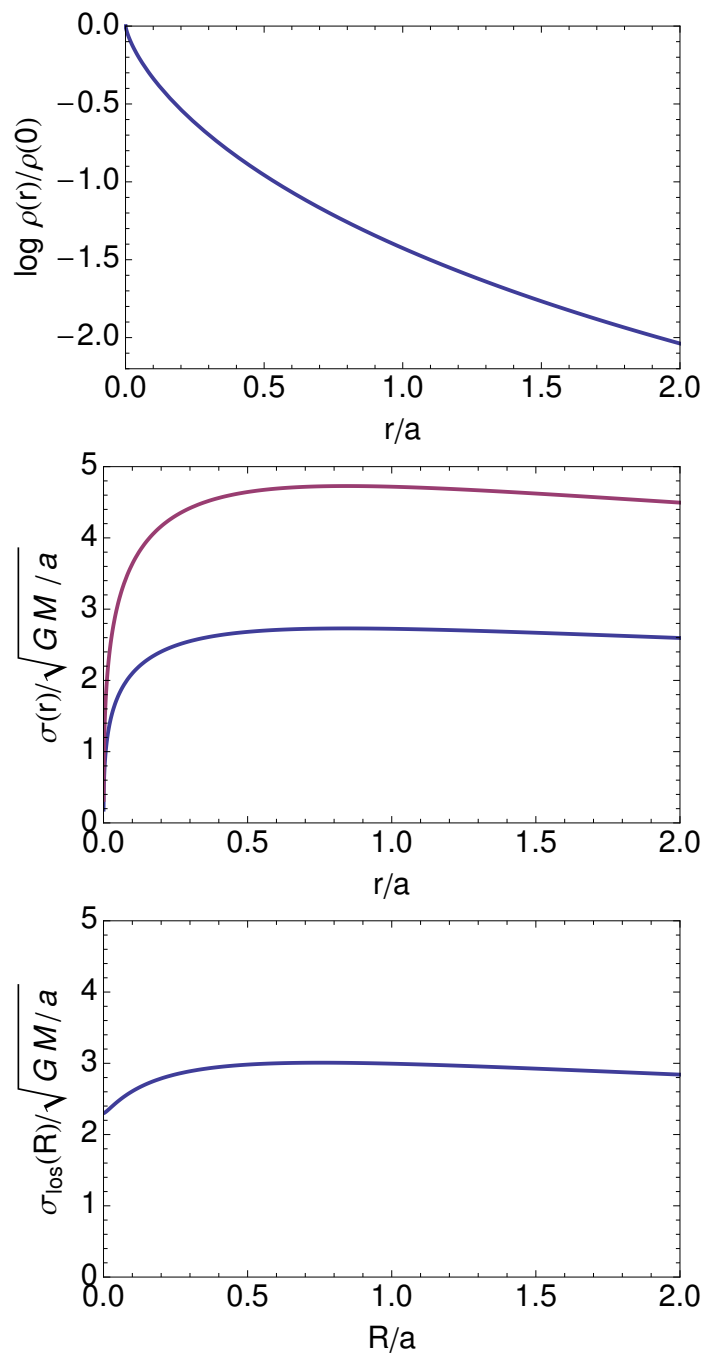

Figure 10: Various moments of the distribution function described in Eq. 40). The top panel shows the predicted stellar density $\nu(r)$ versus distance (in units of the scale $a$ ). The central panel shows the 2nd velocity moments in the radial (blue) and tangential (red) directions. In the bottom panel we plot the $\sigma_{\text {los }}$ profile. Note that this example shows that it is possible for the system to have a relatively flat velocity dispersion profile, even if $\sigma_{r}=0$ at the centre. Moreover, a $\gamma_{*, 0}=0$ stellar profile can be embedded in a cuspy NFW halo, without having an isotropic velocity dispersion at the centre, since here $\beta=-0.5$. 

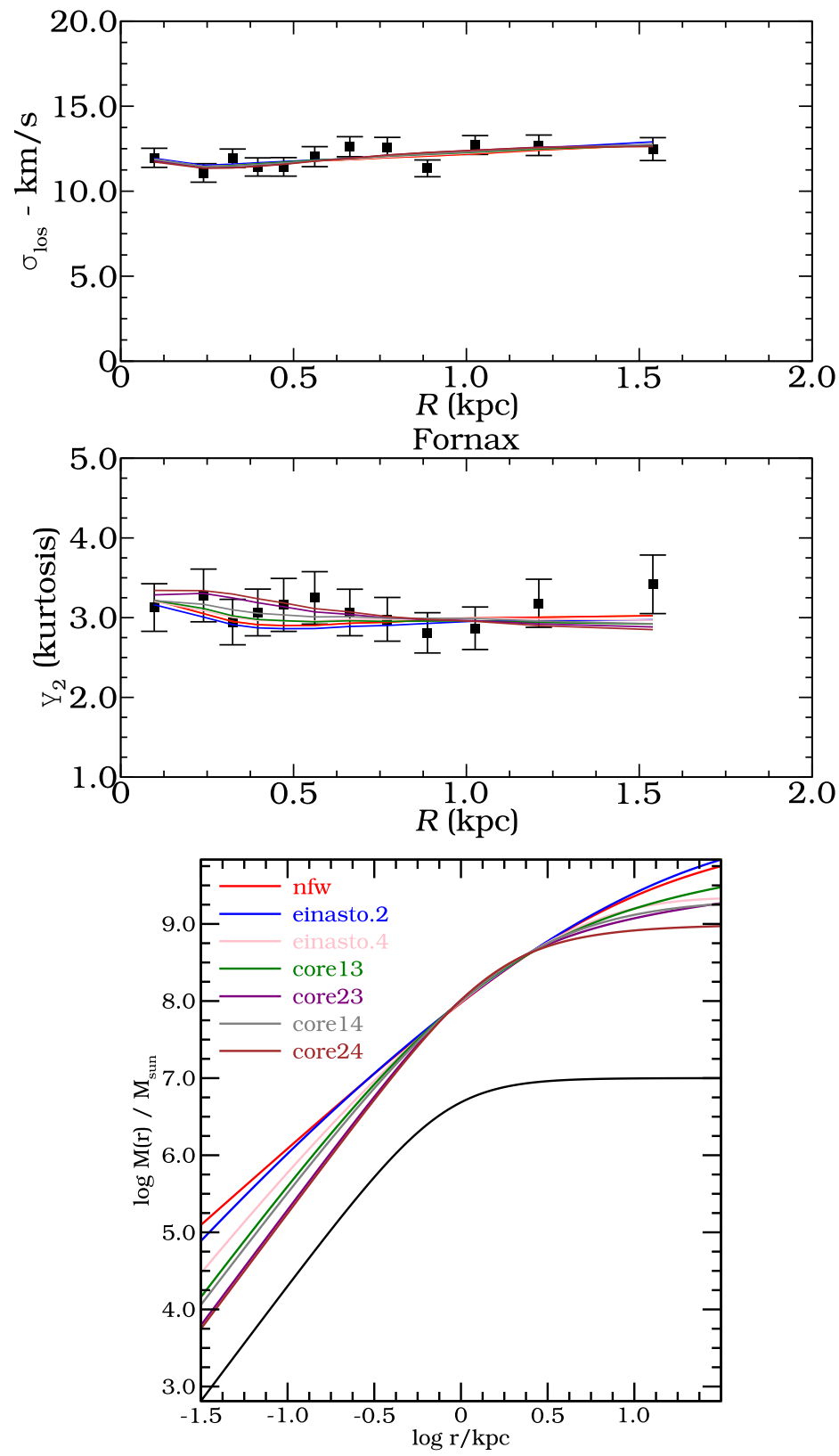

Figure 11: The top and middle panels show the velocity dispersion profiles and kurtosis for Fornax (squares with error bars). The various curves correspond to the best fit models obtained using Schwarzschild's method of orbit superposition. The mass distribution of these models is shown in the bottom panel, and clearly shows that the various models are effectively indistinguishable within a distance range that goes from slightly less than $r_{1 / 2}$ up-to the last measured data point. (From Breddels and Helmi, 2013). 


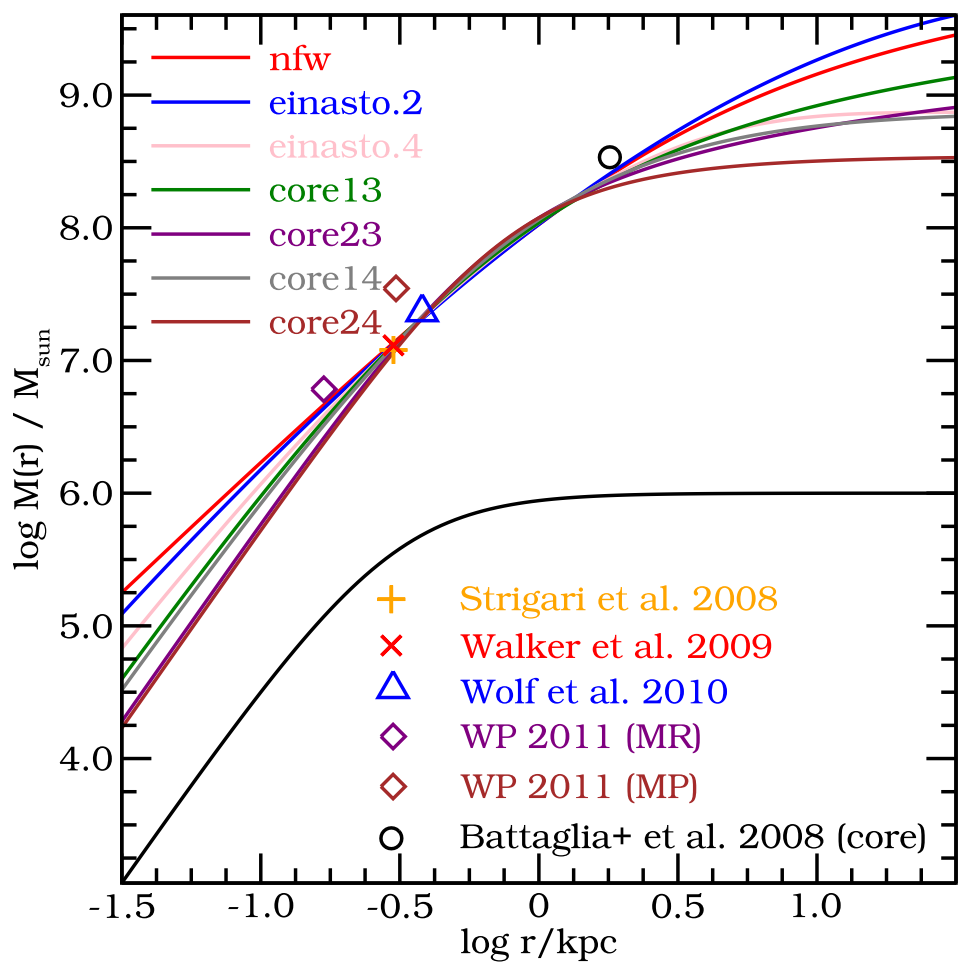

Figure 12: Mass distribution $M(r)$ for Scl derived by Breddels and Helmi (2013). The various curves represent the best fit models obtained using Schwarzschild's orbit-based method. As in the case of Fnx, the various density profiles appear indistinguishable from one another. We have overlaid various measurements for the mass at different radii, including those obtained considering separately the metal-rich and metal-poor populations by Battaglia et al. (2008a), and by estimating separately $M_{1 / 2}$ for the metal-rich (MR) and metal-poor (MP) component in Walker and Peñarrubia (2011). 
An, J. H., Evans, N. W., May 2011. Modified virial formulae and the theory of mass estimators. MNRAS 413, 1744-1752.

Armandroff, T. E., Da Costa, G. S., Oct. 1986. The radial velocity, velocity dispersion, and mass-to-light ratio of the Sculptor dwarf galaxy. AJ 92, $777-786$.

Armandroff, T. E., Olszewski, E. W., Pryor, C., Nov. 1995. The Mass-ToLight Ratios of the Draco and Ursa Minor Dwarf Spheroidal Galaxies.I. Radial Velocities from Multifiber Spectroscopy. AJ 110, 2131.

Battaglia, G., Helmi, A., Tolstoy, E., Irwin, M., Hill, V., Jablonka, P., Jul. 2008a. The Kinematic Status and Mass Content of the Sculptor Dwarf Spheroidal Galaxy. ApJL 681, L13-L16.

Battaglia, G., Irwin, M., Tolstoy, E., de Boer, T., Mateo, M., Dec. 2012. The Extensive Age Gradient of the Carina Dwarf Galaxy. ApJL 761, L31.

Battaglia, G., Irwin, M., Tolstoy, E., Hill, V., Helmi, A., Letarte, B., Jablonka, P., Jan. 2008b. Analysis and calibration of CaII triplet spectroscopy of red giant branch stars from VLT/FLAMES observations. MNRAS 383, 183-199.

Battaglia, G., Starkenburg, E., Mar. 2012. Cleaning spectroscopic samples of stars in nearby dwarf galaxies. The use of the nIR $\mathrm{Mg}$ I line to weed out Milky Way contaminants. A\&A 539, A123.

Battaglia, G., Tolstoy, E., Helmi, A., Irwin, M., Parisi, P., Hill, V., Jablonka, P., Feb. 2011. Study of the Sextans dwarf spheroidal galaxy from the DART Ca II triplet survey. MNRAS 411, 1013-1034.

Battaglia, G., Tolstoy, E., Helmi, A., Irwin, M. J., Letarte, B., Jablonka, P., Hill, V., Venn, K. A., Shetrone, M. D., Arimoto, N., Primas, F., Kaufer, A., Francois, P., Szeifert, T., Abel, T., Sadakane, K., Nov. 2006. The DART imaging and CaT survey of the Fornax dwarf spheroidal galaxy. A\&A 459, 423-440.

Belokurov, V., Zucker, D. B., Evans, N. W., Kleyna, J. T., Koposov, S., Hodgkin, S. T., Irwin, M. J., Gilmore, G., Wilkinson, M. I., Fellhauer, M., Bramich, D. M., Hewett, P. C., Vidrih, S., De Jong, J. T. A., Smith, J. A., Rix, H.-W., Bell, E. F., Wyse, R. F. G., Newberg, H. J., Mayeur, P. A., 
Yanny, B., Rockosi, C. M., Gnedin, O. Y., Schneider, D. P., Beers, T. C., Barentine, J. C., Brewington, H., Brinkmann, J., Harvanek, M., Kleinman, S. J., Krzesinski, J., Long, D., Nitta, A., Snedden, S. A., Jan. 2007. Cats and Dogs, Hair and a Hero: A Quintet of New Milky Way Companions. ApJ 654, 897-906.

Belokurov, V., Zucker, D. B., Evans, N. W., Wilkinson, M. I., Irwin, M. J., Hodgkin, S., Bramich, D. M., Irwin, J. M., Gilmore, G., Willman, B., Vidrih, S., Newberg, H. J., Wyse, R. F. G., Fellhauer, M., Hewett, P. C., Cole, N., Bell, E. F., Beers, T. C., Rockosi, C. M., Yanny, B., Grebel, E. K., Schneider, D. P., Lupton, R., Barentine, J. C., Brewington, H., Brinkmann, J., Harvanek, M., Kleinman, S. J., Krzesinski, J., Long, D., Nitta, A., Smith, J. A., Snedden, S. A., Aug. 2006. A Faint New Milky Way Satellite in Bootes. ApJL 647, L111-L114.

Benson, A. J., Frenk, C. S., Lacey, C. G., Baugh, C. M., Cole, S., Jun. 2002. The effects of photoionization on galaxy formation - II. Satellite galaxies in the Local Group. MNRAS 333, 177-190.

Bertone, G., Hooper, D., Silk, J., Jan. 2005. Particle dark matter: evidence, candidates and constraints. Physics Rep. 405, 279-390.

Binney, J., Tremaine, S., 2008. Galactic Dynamics: Second Edition. Princeton University Press.

Boylan-Kolchin, M., Bullock, J. S., Kaplinghat, M., Jul. 2011. Too big to fail? The puzzling darkness of massive Milky Way subhaloes. MNRAS 415, L40-L44.

Breddels, M. A., Helmi, A., Mar. 2013. Model comparison of dark matter profiles in local dSphs. arXiv:13XX.

Breddels, M. A., Helmi, A., van den Bosch, R. C. E., van de Ven, G., Battaglia, G., May 2012. Orbit-based dynamical models of the Sculptor dSph galaxy. arXiv:1205.4721.

Bullock, J. S., Kolatt, T. S., Sigad, Y., Somerville, R. S., Kravtsov, A. V., Klypin, A. A., Primack, J. R., Dekel, A., Mar. 2001. Profiles of dark haloes: evolution, scatter and environment. MNRAS 321, 559-575. 
Bullock, J. S., Kravtsov, A. V., Weinberg, D. H., Aug. 2000. Reionization and the Abundance of Galactic Satellites. ApJ 539, 517-521.

Busha, M. T., Evrard, A. E., Adams, F. C., Aug. 2007. The Asymptotic Form of Cosmic Structure: Small-Scale Power and Accretion History. ApJ $665,1-13$.

Cappellari, M., Oct. 2008. Measuring the inclination and mass-to-light ratio of axisymmetric galaxies via anisotropic Jeans models of stellar kinematics. MNRAS 390, 71-86.

Chanamé, J., Kleyna, J., van der Marel, R., Aug. 2008. Constraining the Mass Profiles of Stellar Systems: Schwarzschild Modeling of Discrete Velocity Data Sets. ApJ 682, 841-860.

Ciotti, L., Morganti, L., Oct. 2010. How general is the global density slopeanisotropy inequality? MNRAS 408, 1070-1074.

Coleman, M. G., Da Costa, G. S., Bland-Hawthorn, J., Sep. 2005. The Absence of Extratidal Structure in the Sculptor Dwarf Spheroidal Galaxy. AJ 130, 1065-1082.

Colín, P., Avila-Reese, V., Valenzuela, O., Oct. 2000. Substructure and Halo Density Profiles in a Warm Dark Matter Cosmology. ApJ 542, 622-630.

Colín, P., Valenzuela, O., Avila-Reese, V., Jan. 2008. On the Structure of Dark Matter Halos at the Damping Scale of the Power Spectrum with and without Relict Velocities. ApJ 673, 203-214.

de Blok, W. J. G., 2010. The Core-Cusp Problem. Advances in Astronomy 2010 .

de Lorenzi, F., Debattista, V. P., Gerhard, O., Sambhus, N., Mar. 2007. NMAGIC: a fast parallel implementation of a $\chi^{2}$-made-to-measure algorithm for modelling observational data. MNRAS 376, 71-88.

de Lorenzi, F., Gerhard, O., Coccato, L., Arnaboldi, M., Capaccioli, M., Douglas, N. G., Freeman, K. C., Kuijken, K., Merrifield, M. R., Napolitano, N. R., Noordermeer, E., Romanowsky, A. J., Debattista, V. P., May 2009. Dearth of dark matter or massive dark halo? Mass-shape-anisotropy degeneracies revealed by NMAGIC dynamical models of the elliptical galaxy NGC 3379. MNRAS 395, 76-96. 
Dejonghe, H., Jan. 1987. A completely analytical family of anisotropic Plummer models. MNRAS 224, 13-39.

den Hartog, R., Katgert, P., Mar. 1996. On the dynamics of the cores of galaxy clusters. MNRAS 279, 349-388.

Di Cintio, A., Knebe, A., Libeskind, N. I., Brook, C., Yepes, G., Gottloeber, S., Hoffman, Y., Apr. 2012. Size matters: the non-universal density profile of subhaloes in SPH simulations and implications for the Milky Way's dSphs. arXiv:1204.0515.

Duquennoy, A., Mayor, M., Aug. 1991. Multiplicity among solar-type stars in the solar neighbourhood. II - Distribution of the orbital elements in an unbiased sample. A\&A 248, 485-524.

Evans, C., Puech, M., Barbuy, B., Bastian, N., Bonifacio, P., Caffau, E., Cuby, J.-G., Dalton, G., Davies, B., Dunlop, J., Flores, H., Hammer, F., Kaper, L., Lemasle, B., Morris, S., Pentericci, L., Petitjean, P., Schaerer, D., Telles, E., Welikala, N., Ziegler, B., Feb. 2013. ELT-MOS White Paper: Science Overview and Requirements. ArXiv e-prints.

Evans, N. W., An, J., Walker, M. G., Feb. 2009. Cores and cusps in the dwarf spheroidals. MNRAS 393, L50-L54.

Faria, D., Feltzing, S., Lundström, I., Gilmore, G., Wahlgren, G. M., Ardeberg, A., Linde, P., Apr. 2007. The usage of Strömgren photometry in studies of local group dwarf spheroidal galaxies. Application to Draco: a new catalogue of Draco members and a study of the metallicity distribution function and radial gradients. A\&A 465, 357-373.

Feng, J. L., Sep. 2010. Dark Matter Candidates from Particle Physics and Methods of Detection. ARA\&A 48, 495-545.

Font, A. S., Benson, A. J., Bower, R. G., Frenk, C. S., Cooper, A., De Lucia, G., Helly, J. C., Helmi, A., Li, Y.-S., McCarthy, I. G., Navarro, J. F., Springel, V., Starkenburg, E., Wang, J., White, S. D. M., Oct. 2011. The population of Milky Way satellites in the $\Lambda$ cold dark matter cosmology. MNRAS 417, 1260-1279.

Geha, M., van der Marel, R. P., Guhathakurta, P., Gilbert, K. M., Kalirai, J., Kirby, E. N., Mar. 2010. Local Group Dwarf Elliptical Galaxies. II. 
Stellar Kinematics to Large Radii in NGC 147 and NGC 185. ApJ 711, 361-373.

Gerhard, O. E., Jun. 1991. A new family of distribution functions for spherical galaxies. MNRAS 250, 812-830.

Gerhard, O. E., Nov. 1993. Line-of-sight velocity profiles in spherical galaxies: breaking the degeneracy between anisotropy and mass. MNRAS 265, 213.

Gilbert, K. M., Guhathakurta, P., Kalirai, J. S., Rich, R. M., Majewski, S. R., Ostheimer, J. C., Reitzel, D. B., Cenarro, A. J., Cooper, M. C., Luine, C., Patterson, R. J., Dec. 2006. A New Method for Isolating M31 Red Giant Stars: The Discovery of Stars out to a Radial Distance of 165 kpc. ApJ 652, 1188-1212.

Gilmore, G., Wilkinson, M. I., Wyse, R. F. G., Kleyna, J. T., Koch, A., Evans, N. W., Grebel, E. K., Jul. 2007. The Observed Properties of Dark Matter on Small Spatial Scales. ApJ 663, 948-959.

Gnedin, N. Y., Kravtsov, A. V., Jul. 2006. Fossils of Reionization in the Local Group. ApJ 645, 1054-1061.

Governato, F., Brook, C., Mayer, L., Brooks, A., Rhee, G., Wadsley, J., Jonsson, P., Willman, B., Stinson, G., Quinn, T., Madau, P., Jan. 2010. Bulgeless dwarf galaxies and dark matter cores from supernova-driven outflows. Nature 463, 203-206.

Guhathakurta, P., Rich, R. M., Reitzel, D. B., Cooper, M. C., Gilbert, K. M., Majewski, S. R., Ostheimer, J. C., Geha, M. C., Johnston, K. V., Patterson, R. J., May 2006. Dynamics and Stellar Content of the Giant Southern Stream in M31. I. Keck Spectroscopy of Red Giant Stars. AJ 131, 24972513 .

Hammer, F., Yang, Y., Flores, H., Puech, M., Mar. 2013. The vast thin plane of M31 co-rotating dwarfs: an additional fossil signature of the M31 merger and of its considerable impact in the whole Local Group. ArXiv e-prints.

Hargreaves, J. C., Gilmore, G., Annan, J. D., Mar. 1996. The influence of binary stars on dwarf spheroidal galaxy kinematics. MNRAS 279, 108-120. 
Hargreaves, J. C., Gilmore, G., Irwin, M. J., Carter, D., Aug. 1994a. A Dynamical Study of the Sextans Dwarf Spheroidal Galaxy. MNRAS 269, 957-+.

Hargreaves, J. C., Gilmore, G., Irwin, M. J., Carter, D., Dec. 1994b. A Dynamical Study of the Ursa-Minor Dwarf Spheroidal Galaxy. MNRAS $271,693$.

Hayashi, K., Chiba, M., Aug. 2012. Probing Non-spherical Dark Halos in the Galactic Dwarf Galaxies. ApJ 755, 145.

Helmi, A., Irwin, M. J., Tolstoy, E., Battaglia, G., Hill, V., Jablonka, P., Venn, K., Shetrone, M., Letarte, B., Arimoto, N., Abel, T., Francois, P., Kaufer, A., Primas, F., Sadakane, K., Szeifert, T., Nov. 2006. A New View of the Dwarf Spheroidal Satellites of the Milky Way from VLT FLAMES: Where Are the Very Metal-poor Stars? ApJL 651, L121-L124.

Helmi, A., Sales, L. V., Starkenburg, E., Starkenburg, T. K., Vera-Ciro, C. A., De Lucia, G., Li, Y.-S., Oct. 2012. Dark Satellites and the Morphology of Dwarf Galaxies. ApJL 758, L5.

Hernquist, L., Jun. 1990. An analytical model for spherical galaxies and bulges. ApJ 356, 359-364.

Hinshaw, G., Larson, D., Komatsu, E., Spergel, D. N., Bennett, C. L., Dunkley, J., Nolta, M. R., Halpern, M., Hill, R. S., Odegard, N., Page, L., Smith, K. M., Weiland, J. L., Gold, B., Jarosik, N., Kogut, A., Limon, M., Meyer, S. S., Tucker, G. S., Wollack, E., Wright, E. L., Dec. 2012. Nine-Year Wilkinson Microwave Anisotropy Probe (WMAP) Observations: Cosmological Parameter Results. ArXiv e-prints.

Ho, N., Geha, M., Munoz, R. R., Guhathakurta, P., Kalirai, J., Gilbert, K. M., Tollerud, E., Bullock, J., Beaton, R. L., Majewski, S. R., Oct. 2012. Stellar Kinematics of the Andromeda II Dwarf Spheroidal Galaxy. ApJ 758, 124.

Hooper, D., Baltz, E. A., Nov. 2008. Strategies for Determining the Nature of Dark Matter. Annual Review of Nuclear and Particle Science 58, 293-314.

Ibata, R. A., Lewis, G. F., Conn, A. R., Irwin, M. J., McConnachie, A. W., Chapman, S. C., Collins, M. L., Fardal, M., Ferguson, A. M. N., Ibata, 
N. G., Mackey, A. D., Martin, N. F., Navarro, J., Rich, R. M., VallsGabaud, D., Widrow, L. M., Jan. 2013. A vast, thin plane of corotating dwarf galaxies orbiting the Andromeda galaxy. Nature 493, 62-65.

Irwin, M., Hatzidimitriou, D., Dec. 1995. Structural parameters for the Galactic dwarf spheroidals. MNRAS 277, 1354-1378.

Jardel, J. R., Gebhardt, K., Feb. 2012. The Dark Matter Density Profile of the Fornax Dwarf. ApJ 746, 89.

Jardel, J. R., Gebhardt, K., Fabricius, M. H., Drory, N., Williams, M. J., Feb. 2013. Measuring Dark Matter Profiles Non-Parametrically in Dwarf Spheroidals: An Application to Draco. ApJ 763, 91.

Kazantzidis, S., Łokas, E. L., Callegari, S., Mayer, L., Moustakas, L. A., Jan. 2011a. On the Efficiency of the Tidal Stirring Mechanism for the Origin of Dwarf Spheroidals: Dependence on the Orbital and Structural Parameters of the Progenitor Disky Dwarfs. ApJ 726, 98.

Kazantzidis, S., Łokas, E. L., Mayer, L., Feb. 2013. Tidal Stirring of Disky Dwarfs with Shallow Dark Matter Density Profiles: Enhanced Transformation into Dwarf Spheroidals. ApJL 764, L29.

Kazantzidis, S., Łokas, E. L., Mayer, L., Knebe, A., Klimentowski, J., Oct. 2011b. Formation of Dwarf Spheroidal Galaxies via Mergers of Disky Dwarfs. ApJL 740, L24.

King, I., Oct. 1962. The structure of star clusters. I. an empirical density law. AJ 67, 471.

Kirby, E. N., Cohen, J. G., Bellazzini, M., May 2012. The Dynamics and Metallicity Distribution of the Distant Dwarf Galaxy VV124. ApJ 751, 46.

Klessen, R. S., Grebel, E. K., Harbeck, D., Jun. 2003. Draco: A Failure of the Tidal Model. ApJ 589, 798-809.

Kleyna, J., Wilkinson, M. I., Evans, N. W., Gilmore, G., Frayn, C., Mar. 2002. Dark matter in dwarf spheroidals - II. Observations and modelling of Draco. MNRAS 330, 792-806. 
Kleyna, J. T., Wilkinson, M. I., Evans, N. W., Gilmore, G., Dec. 2001. First Clear Signature of an Extended Dark Matter Halo in the Draco Dwarf Spheroidal. ApJL 563, L115-L118.

Kleyna, J. T., Wilkinson, M. I., Evans, N. W., Gilmore, G., Nov. 2004. A photometrically and kinematically distinct core in the Sextans dwarf spheroidal galaxy. MNRAS 354, L66-L72.

Klimentowski, J., Łokas, E. L., Kazantzidis, S., Mayer, L., Mamon, G. A., Prada, F., Dec. 2009. The orientation and kinematics of inner tidal tails around dwarf galaxies orbiting the Milky Way. MNRAS 400, 2162-2168.

Klimentowski, J., Łokas, E. L., Kazantzidis, S., Prada, F., Mayer, L., Mamon, G. A., Jun. 2007. Mass modelling of dwarf spheroidal galaxies: the effect of unbound stars from tidal tails and the Milky Way. MNRAS 378, 353-368.

Klypin, A., Kravtsov, A. V., Valenzuela, O., Prada, F., Sep. 1999. Where Are the Missing Galactic Satellites? ApJ 522, 82-92.

Koch, A., Grebel, E. K., Kleyna, J. T., Wilkinson, M. I., Harbeck, D. R., Gilmore, G. F., Wyse, R. F. G., Evans, N. W., Jan. 2007a. Complexity on Small Scales. II. Metallicities and Ages in the Leo II Dwarf Spheroidal Galaxy. AJ 133, 270-283.

Koch, A., Grebel, E. K., Wyse, R. F. G., Kleyna, J. T., Wilkinson, M. I., Harbeck, D. R., Gilmore, G. F., Evans, N. W., Feb. 2006. Complexity on Small Scales: The Metallicity Distribution of the Carina Dwarf Spheroidal Galaxy. AJ 131, 895-911.

Koch, A., Kleyna, J. T., Wilkinson, M. I., Grebel, E. K., Gilmore, G. F., Evans, N. W., Wyse, R. F. G., Harbeck, D. R., Aug. 2007b. Stellar Kinematics in the Remote Leo II Dwarf Spheroidal Galaxy-Another Brick in the Wall. AJ 134, 566-578.

Koch, A., Wilkinson, M. I., Kleyna, J. T., Gilmore, G. F., Grebel, E. K., Mackey, A. D., Evans, N. W., Wyse, R. F. G., Mar. 2007c. Stellar Kinematics and Metallicities in the Leo I Dwarf Spheroidal Galaxy-Wide-Field Implications for Galactic Evolution. ApJ 657, 241-261. 
Koposov, S., Belokurov, V., Evans, N. W., Hewett, P. C., Irwin, M. J., Gilmore, G., Zucker, D. B., Rix, H., Fellhauer, M., Bell, E. F., Glushkova, E. V., Oct. 2008. The Luminosity Function of the Milky Way Satellites. ApJ 686, 279-291.

Koposov, S. E., Gilmore, G., Walker, M. G., Belokurov, V., Wyn Evans, N., Fellhauer, M., Gieren, W., Geisler, D., Monaco, L., Norris, J. E., Okamoto, S., Peñarrubia, J., Wilkinson, M., Wyse, R. F. G., Zucker, D. B., Aug. 2011. Accurate Stellar Kinematics at Faint Magnitudes: Application to the Boötes I Dwarf Spheroidal Galaxy. ApJ 736, 146.

Koposov, S. E., Yoo, J., Rix, H.-W., Weinberg, D. H., Macciò, A. V., Escudé, J. M., May 2009. A Quantitative Explanation of the Observed Population of Milky Way Satellite Galaxies. ApJ 696, 2179-2194.

Kuhlen, M., Diemand, J., Madau, P., Dec. 2007. The Shapes, Orientation, and Alignment of Galactic Dark Matter Subhalos. ApJ 671, 1135-1146.

Leaman, R., Venn, K. A., Brooks, A. M., Battaglia, G., Cole, A. A., Ibata, R. A., Irwin, M. J., McConnachie, A. W., Mendel, J. T., Tolstoy, E., May 2012. The Resolved Structure and Dynamics of an Isolated Dwarf Galaxy: A VLT and Keck Spectroscopic Survey of WLM. ApJ 750, 33.

Li, Y.-S., De Lucia, G., Helmi, A., Jan. 2010. On the nature of the Milky Way satellites. MNRAS 401, 2036-2052.

Łokas, E. L., Oct. 2001. Velocity dispersions of dwarf spheroidal galaxies: dark matter versus MOND. MNRAS 327, L21-L26.

Łokas, E. L., Jul. 2002. Dark matter distribution in dwarf spheroidal galaxies. MNRAS 333, 697-708.

Łokas, E. L., Mar. 2009. The mass and velocity anisotropy of the Carina, Fornax, Sculptor and Sextans dwarf spheroidal galaxies. MNRAS 394, L102L106.

Łokas, E. L., Kazantzidis, S., Klimentowski, J., Mayer, L., Callegari, S., Jan. 2010. The Stellar Structure and Kinematics of Dwarf Spheroidal Galaxies Formed by Tidal Stirring. ApJ 708, 1032-1047. 
Łokas, E. L., Klimentowski, J., Kazantzidis, S., Mayer, L., Oct. 2008. The anatomy of Leo I: how tidal tails affect the kinematics. MNRAS 390, 625634 .

Łokas, E. L., Mamon, G. A., Aug. 2003a. Dark matter distribution in the Coma cluster from galaxy kinematics: breaking the mass-anisotropy degeneracy. MNRAS 343, 401-412.

Łokas, E. L., Mamon, G. A., Aug. 2003b. Dark matter distribution in the Coma cluster from galaxy kinematics: breaking the mass-anisotropy degeneracy. MNRAS 343, 401-412.

Łokas, E. L., Mamon, G. A., Prada, F., Nov. 2005. Dark matter distribution in the Draco dwarf from velocity moments. MNRAS 363, 918-928.

Long, R. J., Mao, S., Jun. 2010. Made-to-measure galaxy models - I. Methodology. MNRAS 405, 301-317.

Long, R. J., Mao, S., Shen, J., Wang, Y., Feb. 2013. Made-to-measure galaxy models - III. Modelling with Milky Way observations. MNRAS 428, 34783486 .

Lovell, M. R., Eke, V., Frenk, C. S., Gao, L., Jenkins, A., Theuns, T., Wang, J., White, S. D. M., Boyarsky, A., Ruchayskiy, O., Mar. 2012. The haloes of bright satellite galaxies in a warm dark matter universe. MNRAS 420, $2318-2324$.

Lynden-Bell, D., 1960. Can spherical clusters rotate? MNRAS 120, 204.

Macciò, A. V., Dutton, A. A., van den Bosch, F. C., Moore, B., Potter, D., Stadel, J., Jun. 2007. Concentration, spin and shape of dark matter haloes: scatter and the dependence on mass and environment. MNRAS $378,55-71$.

Macciò, A. V., Paduroiu, S., Anderhalden, D., Schneider, A., Moore, B., Aug. 2012. Cores in warm dark matter haloes: a Catch 22 problem. MNRAS $424,1105-1112$.

Macciò, A. V., Paduroiu, S., Anderhalden, D., Schneider, A., Moore, B., Feb. 2013. Erratum: Cores in warm dark matter haloes: a Catch 22 problem. MNRAS 428, 3715-3716. 
Majewski, S. R., Frinchaboy, P. M., Kunkel, W. E., Link, R., Muñoz, R. R., Ostheimer, J. C., Palma, C., Patterson, R. J., Geisler, D., Dec. 2005. Exploring Halo Substructure with Giant Stars. VI. Extended Distributions of Giant Stars around the Carina Dwarf Spheroidal Galaxy: How Reliable Are They? AJ 130, 2677-2700.

Majewski, S. R., Ostheimer, J. C., Patterson, R. J., Kunkel, W. E., Johnston, K. V., Geisler, D., Feb. 2000. Exploring Halo Substructure with Giant Stars. II. Mapping the Extended Structure of the Carina Dwarf Spheroidal Galaxy. AJ 119, 760-776.

Mamon, G. A., Biviano, A., Boué, G., Mar. 2013. MAMPOSSt: Modelling Anisotropy and Mass Profiles of Observed Spherical Systems - I. Gaussian 3D velocities. MNRAS 429, 3079-3098.

Martin, N. F., Ibata, R. A., Chapman, S. C., Irwin, M., Lewis, G. F., Sep. 2007. A Keck/DEIMOS spectroscopic survey of faint Galactic satellites: searching for the least massive dwarf galaxies. MNRAS 380, 281-300.

Martin, N. F., McConnachie, A. W., Irwin, M., Widrow, L. M., Ferguson, A. M. N., Ibata, R. A., Dubinski, J., Babul, A., Chapman, S., Fardal, M., Lewis, G. F., Navarro, J., Rich, R. M., Nov. 2009. PAndAS' CUBS: Discovery of Two New Dwarf Galaxies in the Surroundings of the Andromeda and Triangulum Galaxies. ApJ 705, 758-765.

Martinez, G. D., Minor, Q. E., Bullock, J., Kaplinghat, M., Simon, J. D., Geha, M., Sep. 2011. A Complete Spectroscopic Survey of the Milky Way Satellite Segue 1: Dark Matter Content, Stellar Membership, and Binary Properties from a Bayesian Analysis. ApJ 738, 55-+.

Martínez-Delgado, D., Alonso-García, J., Aparicio, A., Gómez-Flechoso, M. A., Mar. 2001. A Tidal Extension in the Ursa Minor Dwarf Spheroidal Galaxy. ApJL 549, L63-L66.

Mateo, M., Nemec, J., Irwin, M., McMahon, R., Mar. 1991a. Deep CCD photometry of the Sextans dwarf spheroidal galaxy. AJ 101, 892-910.

Mateo, M., Olszewski, E., Welch, D. L., Fischer, P., Kunkel, W., Sep. 1991b. A kinematic study of the Fornax dwarf spheroidal galaxy. AJ 102, 914-926. 
Mateo, M., Olszewski, E. W., Vogt, S. S., Keane, M. J., Nov. 1998. The Internal Kinematics of the Leo I Dwarf Spheroidal Galaxy: Dark Matter at the Fringe of the Milky Way. AJ 116, 2315-2327.

Mateo, M., Olszewski, E. W., Walker, M. G., Mar. 2008. The Velocity Dispersion Profile of the Remote Dwarf Spheroidal Galaxy Leo I: A Tidal Hit and Run? ApJ 675, 201-233.

Mayer, L., Governato, F., Colpi, M., Moore, B., Quinn, T., Wadsley, J., Stadel, J., Lake, G., Feb. 2001. Tidal Stirring and the Origin of Dwarf Spheroidals in the Local Group. ApJL 547, L123-L127.

McConnachie, A. W., Côté, P., Oct. 2010. Revisiting the Influence of Unidentified Binaries on Velocity Dispersion Measurements in Ultra-faint Stellar Systems. ApJL 722, L209-L214.

McConnachie, A. W., Irwin, M. J., Ibata, R. A., Dubinski, J., Widrow, L. M., Martin, N. F., Côté, P., Dotter, A. L., Navarro, J. F., Ferguson, A. M. N., Puzia, T. H., Lewis, G. F., Babul, A., Barmby, P., Bienaymé, O., Chapman, S. C., Cockcroft, R., Collins, M. L. M., Fardal, M. A., Harris, W. E., Huxor, A., Mackey, A. D., Peñarrubia, J., Rich, R. M., Richer, H. B., Siebert, A., Tanvir, N., Valls-Gabaud, D., Venn, K. A., Sep. 2009. The remnants of galaxy formation from a panoramic survey of the region around M31. Nature 461, 66-69.

Merrifield, M. R., Kent, S. M., May 1990. Fourth moments and the dynamics of spherical systems. AJ 99, 1548-1557.

Merritt, D., Aug. 1993. Dynamical mapping of hot stellar systems. ApJ 413, 79-94.

Merritt, D., Saha, P., May 1993. Mapping spherical potentials with discrete radial velocities. ApJ 409, 75-90.

Metz, M., Kroupa, P., Jerjen, H., Apr. 2009. Discs of satellites: the new dwarf spheroidals. MNRAS 394, 2223-2228.

Minor, Q. E., Feb. 2013. Binary populations in Milky Way satellite galaxies: constraints from multi-epoch data in the Carina, Fornax, Sculptor and Sextans dwarf spheroidal galaxies. ArXiv e-prints. 
Minor, Q. E., Martinez, G., Bullock, J., Kaplinghat, M., Trainor, R., Oct. 2010. Correcting Velocity Dispersions of Dwarf Spheroidal Galaxies for Binary Orbital Motion. ApJ 721, 1142-1157.

Moore, B., Ghigna, S., Governato, F., Lake, G., Quinn, T., Stadel, J., Tozzi, P., Oct. 1999. Dark Matter Substructure within Galactic Halos. ApJL 524, L19-L22.

Morganti, L., Gerhard, O., Coccato, L., Martinez-Valpuesta, I., Arnaboldi, M., Mar. 2013. Elliptical galaxies with rapidly decreasing velocity dispersion profiles: NMAGIC models and dark halo parameter estimates for NGC 4494. ArXiv e-prints.

Morrison, H. L., Olszewski, E. W., Mateo, M., Norris, J. E., Harding, P., Dohm-Palmer, R. C., Freeman, K. C., Jan. 2001. Mapping the Galactic Halo. IV. Finding Distant Giants Reliably with the Washington System. AJ 121, 283-294.

Muñoz, R. R., Frinchaboy, P. M., Majewski, S. R., Kuhn, J. R., Chou, M.Y., Palma, C., Sohn, S. T., Patterson, R. J., Siegel, M. H., Oct. 2005. Exploring Halo Substructure with Giant Stars: The Velocity Dispersion Profiles of the Ursa Minor and Draco Dwarf Spheroidal Galaxies at Large Angular Separations. ApJL 631, L137-L141.

Muñoz, R. R., Majewski, S. R., Johnston, K. V., May 2008. Modeling the Structure and Dynamics of Dwarf Spheroidal Galaxies with Dark Matter and Tides. ApJ 679, 346-372.

Muñoz, R. R., Majewski, S. R., Zaggia, S., Kunkel, W. E., Frinchaboy, P. M., Nidever, D. L., Crnojevic, D., Patterson, R. J., Crane, J. D., Johnston, K. V., Sohn, S. T., Bernstein, R., Shectman, S., Sep. 2006. Exploring Halo Substructure with Giant Stars. XI. The Tidal Tails of the Carina Dwarf Spheroidal Galaxy and the Discovery of Magellanic Cloud Stars in the Carina Foreground. ApJ 649, 201-223.

Navarro, J. F., Eke, V. R., Frenk, C. S., Dec. 1996a. The cores of dwarf galaxy haloes. MNRAS 283, L72-L78.

Navarro, J. F., Frenk, C. S., White, S. D. M., May 1996b. The Structure of Cold Dark Matter Halos. ApJ 462, 563. 
Navarro, J. F., Frenk, C. S., White, S. D. M., Dec. 1997. A Universal Density Profile from Hierarchical Clustering. ApJ 490, 493.

Navarro, J. F., Ludlow, A., Springel, V., Wang, J., Vogelsberger, M., White, S. D. M., Jenkins, A., Frenk, C. S., Helmi, A., Feb. 2010. The diversity and similarity of simulated cold dark matter haloes. MNRAS 402, 21-34.

Odenkirchen, M., Grebel, E. K., Harbeck, D., Dehnen, W., Rix, H.-W., Newberg, H. J., Yanny, B., Holtzman, J., Brinkmann, J., Chen, B., Csabai, I., Hayes, J. J. E., Hennessy, G., Hindsley, R. B., Ivezić, Ž., Kinney, E. K., Kleinman, S. J., Long, D., Lupton, R. H., Neilsen, E. H., Nitta, A., Snedden, S. A., York, D. G., Nov. 2001. New Insights on the Draco Dwarf Spheroidal Galaxy from the Sloan Digital Sky Survey: A Larger Radius and No Tidal Tails. AJ 122, 2538-2553.

Olszewski, E. W., Pryor, C., Armandroff, T. E., Feb. 1996. The Mass-to-Light Ratios of the Draco and Ursa Minor Dwarf Spheroidal Galaxies. II. The Binary Population and its Effects on the Measured Velocity Dispersions of Dwarf Spheroidals. AJ 111, 750.

Palma, C., Majewski, S. R., Siegel, M. H., Patterson, R. J., Ostheimer, J. C., Link, R., Mar. 2003. Exploring Halo Substructure with Giant Stars. IV. The Extended Structure of the Ursa Minor Dwarf Spheroidal Galaxy. AJ $125,1352-1372$.

Pasquini, L., Avila, G., Blecha, A., Cacciari, C., Cayatte, V., Colless, M., Damiani, F., de Propris, R., Dekker, H., di Marcantonio, P., Farrell, T., Gillingham, P., Guinouard, I., Hammer, F., Kaufer, A., Hill, V., Marteaud, M., Modigliani, A., Mulas, G., North, P., Popovic, D., Rossetti, E., Royer, F., Santin, P., Schmutzer, R., Simond, G., Vola, P., Waller, L., Zoccali, M., Dec. 2002. Installation and commissioning of FLAMES, the VLT Multifibre Facility. The Messenger 110, 1-9.

Peñarrubia, J., McConnachie, A. W., Navarro, J. F., Jan. 2008a. The Cold Dark Matter Halos of Local Group Dwarf Spheroidals. ApJ 672, 904-913.

Peñarrubia, J., Navarro, J. F., McConnachie, A. W., Jan. 2008b. The Tidal Evolution of Local Group Dwarf Spheroidals. ApJ 673, 226-240. 
Peñarrubia, J., Pontzen, A., Walker, M. G., Koposov, S. E., Nov. 2012. The Coupling between the Core/Cusp and Missing Satellite Problems. ApJL 759, L42.

Piatek, S., Pryor, C., Jul. 2008. Measuring proper motions of galactic dwarf galaxies with Hubble Space Telescope. In: Jin, W. J., Platais, I., Perryman, M. A. C. (Eds.), IAU Symposium. Vol. 248 of IAU Symposium. pp. 244247.

Piatek, S., Pryor, C., Bristow, P., Olszewski, E. W., Harris, H. C., Mateo, M., Minniti, D., Tinney, C. G., Mar. 2006. Proper Motions of Dwarf Spheroidal Galaxies from Hubble Space Telescope Imaging. IV. Measurement for Sculptor. AJ 131, 1445-1460.

Pontzen, A., Governato, F., Apr. 2012. How supernova feedback turns dark matter cusps into cores. MNRAS 421, 3464-3471.

Prusti, T., Feb. 2011. General status of the Gaia mission and expected performance. In: EAS Publications Series. Vol. 45 of EAS Publications Series. pp. $9-14$.

Queloz, D., Dubath, P., Pasquini, L., Aug. 1995. A kinematic study of the Sculptor dwarf spheroidal galaxy. A\&A 300, 31.

Rashkov, V., Madau, P., Kuhlen, M., Diemand, J., Feb. 2012. On the Assembly of the Milky Way Dwarf Satellites and Their Common Mass Scale. ApJ 745, 142.

Read, J. I., Gilmore, G., Jan. 2005. Mass loss from dwarf spheroidal galaxies: the origins of shallow dark matter cores and exponential surface brightness profiles. MNRAS 356, 107-124.

Read, J. I., Wilkinson, M. I., Evans, N. W., Gilmore, G., Kleyna, J. T., Mar. 2006. The importance of tides for the Local Group dwarf spheroidals. MNRAS 367, 387-399.

Revaz, Y., Jablonka, P., Feb. 2012. The dynamical and chemical evolution of dwarf spheroidal galaxies with GEAR. A\&A 538, A82.

Revaz, Y., Jablonka, P., Sawala, T., Hill, V., Letarte, B., Irwin, M., Battaglia, G., Helmi, A., Shetrone, M. D., Tolstoy, E., Venn, K. A., Jul. 
2009. The dynamical and chemical evolution of dwarf spheroidal galaxies. A\&A 501, 189-206.

Richstone, D. O., Tremaine, S., Nov. 1984. A general method for constructing spherical galaxy models. ApJ 286, 27-37.

Rix, H., de Zeeuw, P. T., Cretton, N., van der Marel, R. P., Carollo, C. M., Oct. 1997. Dynamical Modeling of Velocity Profiles: The Dark Halo around the Elliptical Galaxy NGC 2434. ApJ 488, 702-+.

Robin, A. C., Reylé, C., Derrière, S., Picaud, S., Oct. 2003. A synthetic view on structure and evolution of the Milky Way. A\&A 409, 523-540.

Sawala, T., Scannapieco, C., Maio, U., White, S., Mar. 2010. Formation of isolated dwarf galaxies with feedback. MNRAS 402, 1599-1613.

Sawala, T., Scannapieco, C., White, S., Feb. 2012. Local Group dwarf galaxies: nature and nurture. MNRAS 420, 1714-1730.

Schiavon, R. P., Barbuy, B., Rossi, S. C. F., Milone, A., Apr. 1997. The Near-Infrared NA i Doublet Feature in M Stars. ApJ 479, 902-+.

Schroyen, J., de Rijcke, S., Valcke, S., Cloet-Osselaer, A., Dejonghe, H., Sep. 2011. Simulations of the formation and evolution of isolated dwarf galaxies - II. Angular momentum as a second parameter. MNRAS 416, 601-617.

Schwarzschild, M., Aug. 1979. A numerical model for a triaxial stellar system in dynamical equilibrium. ApJ 232, 236-247.

Schweitzer, A. E., Cudworth, K. M., Majewski, S. R., Suntzeff, N. B., Dec. 1995. The Absolute Proper Motion and a Membership Survey of the Sculptor Dwarf Spheroidal Galaxy. AJ 110, 2747-+.

Seljak, U., Makarov, A., McDonald, P., Trac, H., Nov. 2006. Can Sterile Neutrinos Be the Dark Matter? Physical Review Letters 97 (19), 191303.

Sersic, J. L., 1968. Atlas de galaxias australes.

Siegel, M. H., Shetrone, M. D., Irwin, M., Jun. 2008. Trimming Down the Willman 1 dSph. AJ 135, 2084-2094. 
Simon, J. D., Geha, M., Nov. 2007. The Kinematics of the Ultra-faint Milky Way Satellites: Solving the Missing Satellite Problem. ApJ 670, 313-331.

Sohn, S. T., Majewski, S. R., Muñoz, R. R., Kunkel, W. E., Johnston, K. V., Ostheimer, J. C., Guhathakurta, P., Patterson, R. J., Siegel, M. H., Cooper, M. C., Jul. 2007. Exploring Halo Substructure with Giant Stars. X. Extended Dark Matter or Tidal Disruption?: The Case for the Leo I Dwarf Spheroidal Galaxy. ApJ 663, 960-989.

Somerville, R. S., Jun. 2002. Can Photoionization Squelching Resolve the Substructure Crisis? ApJL 572, L23-L26.

Springel, V., Wang, J., Vogelsberger, M., Ludlow, A., Jenkins, A., Helmi, A., Navarro, J. F., Frenk, C. S., White, S. D. M., Dec. 2008. The Aquarius Project: the subhaloes of galactic haloes. MNRAS 391, 1685-1711.

Starkenburg, E., Helmi, A., De Lucia, G., Li, Y.-S., Navarro, J. F., Font, A. S., Frenk, C. S., Springel, V., Vera-Ciro, C. A., White, S. D. M., Feb. 2013. The satellites of the Milky Way - insights from semi-analytic modelling in a $\Lambda$ CDM cosmology. MNRAS 429, 725-743.

Starkenburg, E., Hill, V., Tolstoy, E., González Hernández, J. I., Irwin, M., Helmi, A., Battaglia, G., Jablonka, P., Tafelmeyer, M., Shetrone, M., Venn, K., de Boer, T., Apr. 2010. The NIR Ca ii triplet at low metallicity. Searching for extremely low-metallicity stars in classical dwarf galaxies. A\&A 513, A34.

Strigari, L. E., Bullock, J. S., Kaplinghat, M., Mar. 2007a. Determining the Nature of Dark Matter with Astrometry. ApJL 657, L1-L4.

Strigari, L. E., Bullock, J. S., Kaplinghat, M., Diemand, J., Kuhlen, M., Madau, P., Nov. 2007b. Redefining the Missing Satellites Problem. ApJ 669, 676-683.

Strigari, L. E., Bullock, J. S., Kaplinghat, M., Simon, J. D., Geha, M., Willman, B., Walker, M. G., Aug. 2008. A common mass scale for satellite galaxies of the Milky Way. Nature 454, 1096-1097.

Strigari, L. E., Frenk, C. S., White, S. D. M., Nov. 2010. Kinematics of Milky Way satellites in a Lambda cold dark matter universe. MNRAS 408, 23642372 . 
Syer, D., Tremaine, S., Sep. 1996. Made-to-measure N-body systems. MNRAS 282, 223-233.

Teyssier, R., Pontzen, A., Dubois, Y., Read, J. I., Mar. 2013. Cusp-core transformations in dwarf galaxies: observational predictions. MNRAS 429, 3068-3078.

Tolstoy, E., Hill, V., Irwin, M., Helmi, A., Battaglia, G., Letarte, B., Venn, K., Jablonka, P., Shetrone, M., Arimoto, N., Abel, T., Primas, F., Kaufer, A., Szeifert, T., Francois, P., Sadakane, K., Mar. 2006. The Dwarf galaxy Abundances and Radial-velocities Team (DART) Large Programme - A Close Look at Nearby Galaxies. The Messenger 123, 33-- .

Tolstoy, E., Hill, V., Tosi, M., Sep. 2009. Star-Formation Histories, Abundances, and Kinematics of Dwarf Galaxies in the Local Group. ARA\&A $47,371-425$.

Tolstoy, E., Irwin, M. J., Helmi, A., Battaglia, G., Jablonka, P., Hill, V., Venn, K. A., Shetrone, M. D., Letarte, B., Cole, A. A., Primas, F., Francois, P., Arimoto, N., Sadakane, K., Kaufer, A., Szeifert, T., Abel, T., Dec. 2004. Two Distinct Ancient Components in the Sculptor Dwarf Spheroidal Galaxy: First Results from the Dwarf Abundances and Radial Velocities Team. ApJL 617, L119-L122.

Valcke, S., de Rijcke, S., Dejonghe, H., Sep. 2008. Simulations of the formation and evolution of isolated dwarf galaxies. MNRAS 389, 1111-1126.

van de Ven, G., van den Bosch, R. C. E., Verolme, E. K., de Zeeuw, P. T., Jan. 2006. The dynamical distance and intrinsic structure of the globular cluster $\omega$ Centauri. A\&A 445, 513-543.

van den Bosch, R. C. E., van de Ven, G., Verolme, E. K., Cappellari, M., de Zeeuw, P. T., Apr. 2008. Triaxial orbit based galaxy models with an application to the (apparent) decoupled core galaxy NGC 4365. MNRAS $385,647-666$.

van der Marel, R. P., Cretton, N., de Zeeuw, P. T., Rix, H.-W., Jan. 1998. Improved Evidence for a Black Hole in M32 from HST/FOS Spectra. II. Axisymmetric Dynamical Models. ApJ 493, 613-+. 
van der Marel, R. P., Franx, M., Apr. 1993. A new method for the identification of non-Gaussian line profiles in elliptical galaxies. ApJ 407, 525-539.

Vera-Ciro, C. A., Helmi, A., Starkenburg, E., Breddels, M. A., Feb. 2012. Not too big, not too small: the dark halos of the dwarf spheroidals in the Milky Way. arXiv:1202.6061.

Vera-Ciro, C. A., Sales, L. V., Helmi, A., Navarro, J. F., Apr. 2013. The shapes of the dark halos... arXiv:13XX.XXXX.

Viel, M., Becker, G. D., Bolton, J. S., Haehnelt, M. G., Rauch, M., Sargent, W. L. W., Feb. 2008. How Cold Is Cold Dark Matter? Small-Scales Constraints from the Flux Power Spectrum of the High-Redshift Lyman- $\alpha$ Forest. Physical Review Letters 100 (4), 041304.

Viel, M., Lesgourgues, J., Haehnelt, M. G., Matarrese, S., Riotto, A., Mar. 2005. Constraining warm dark matter candidates including sterile neutrinos and light gravitinos with WMAP and the Lyman- $\alpha$ forest. Phys. Rev. D 71 (6), 063534.

Walker, M. G., May 2012. Dark Matter in the Milky Way's Dwarf Spheroidal Satellites. arXiv:1205.0311.

Walker, M. G., Mateo, M., Olszewski, E. W., Dec. 2008. Systemic Proper Motions of Milky Way Satellites from Stellar Redshifts: The Carina, Fornax, Sculptor, and Sextans Dwarf Spheroidals. ApJL 688, L75-L78.

Walker, M. G., Mateo, M., Olszewski, E. W., Feb. 2009a. Stellar Velocities in the Carina, Fornax, Sculptor, and Sextans dSph Galaxies: Data From the Magellan/MMFS Survey. AJ 137, 3100-3108.

Walker, M. G., Mateo, M., Olszewski, E. W., Bernstein, R., Sen, B., Woodroofe, M., Aug. 2007a. The Michigan/MIKE Fiber System Survey of Stellar Radial Velocities in Dwarf Spheroidal Galaxies: Acquisition and Reduction of Data. ApJS 171, 389-418.

Walker, M. G., Mateo, M., Olszewski, E. W., Bernstein, R., Wang, X., Woodroofe, M., Apr. 2006. Internal Kinematics of the Fornax Dwarf Spheroidal Galaxy. AJ 131, 2114-2139. 
Walker, M. G., Mateo, M., Olszewski, E. W., Gnedin, O. Y., Wang, X., Sen, B., Woodroofe, M., Sep. 2007b. Velocity Dispersion Profiles of Seven Dwarf Spheroidal Galaxies. ApJL 667, L53-L56.

Walker, M. G., Mateo, M., Olszewski, E. W., Peñarrubia, J., Wyn Evans, N., Gilmore, G., Oct. 2009b. A Universal Mass Profile for Dwarf Spheroidal Galaxies? ApJ 704, 1274-1287.

Walker, M. G., Mateo, M., Olszewski, E. W., Peñarrubia, J., Wyn Evans, N., Gilmore, G., Feb. 2010. Erratum: "A Universal Mass Profile For Dwarf Spheroidal Galaxies?" ¡A href="/abs/2009ApJ...704.1274W" ¿(2009, ApJ, 704, 1274)i/Ai. ApJ 710, 886-890.

Walker, M. G., Mateo, M., Olszewski, E. W., Sen, B., Woodroofe, M., Feb. 2009c. Clean Kinematic Samples in Dwarf Spheroidals: An Algorithm for Evaluating Membership and Estimating Distribution Parameters When Contamination is Present. AJ 137, 3109-3138.

Walker, M. G., Peñarrubia, J., Nov. 2011. A Method for Measuring (Slopes of) the Mass Profiles of Dwarf Spheroidal Galaxies. ApJ 742, 20.

Wang, J., Frenk, C. S., Navarro, J. F., Gao, L., Sawala, T., Aug. 2012. The missing massive satellites of the Milky Way. MNRAS 424, 2715-2721.

Wang, J., White, S. D. M., Jun. 2009. Are mergers responsible for universal halo properties? MNRAS 396, 709-717.

Westfall, K. B., Majewski, S. R., Ostheimer, J. C., Frinchaboy, P. M., Kunkel, W. E., Patterson, R. J., Link, R., Jan. 2006. Exploring Halo Substructure with Giant Stars. VIII. The Extended Structure of the Sculptor Dwarf Spheroidal Galaxy. AJ 131, 375-406.

Wilkinson, M. I., Kleyna, J., Evans, N. W., Gilmore, G., Mar. 2002. Dark matter in dwarf spheroidals - I. Models. MNRAS 330, 778-791.

Wilkinson, M. I., Kleyna, J. T., Evans, N. W., Gilmore, G. F., Irwin, M. J., Grebel, E. K., Aug. 2004. Kinematically Cold Populations at Large Radii in the Draco and Ursa Minor Dwarf Spheroidal Galaxies. ApJL 611, L21L24. 